\title{
Growth of III-V semiconductor nanowires and their heterostructures
}

\author{
Ang $\mathrm{Li}^{1 *}$, Jin Zou ${ }^{2}$ and Xiaodong Han ${ }^{1}$
}

\begin{abstract}
In this paper, we present a review about recent progress on the growth of III-V semiconductor homo- and heterostructured nanowires. We will first deliver a general discussion on the crystal structure and the conventional growth mechanism of one dimensional nanowires. Then we provide a review about most widely used growth techniques, sample preparation and the cutting edge characterization techniques including advanced electron microscopy, in situ electron diffraction, micro-Raman spectroscopy, and atom probe tomography. In the end, the growth of different heteostructured III-V semiconductor nanowires will be reviewed. We will focus on the morphology dependence, temperature influence, and III/ $\mathrm{V}$ flux ratio dependent growth. The perspective and an outlook of this field is discussed in order to foresee the future of the fundamental research and application of these one dimensional nanostructures.
\end{abstract}

Keywords III-V semiconductor heterostructure, nanowire, advanced charactrization, epitaxy growth

\section{INTRODUCTION}

Booming of integrated circuit technologies has been accomplished during the past five decades resulting in electronic devices with higher device density and lower power consumption [1]. As the devices reach the sub-100 nm scale, conventional scaling methods are facing technological and fundamental challenges. A route to overcome these difficulties is to employ structures with their size in the range of 1-100 $\mathrm{nm}$ at least in one dimension, so-called "nanostructures". Due to their large surface-bulk ratio and size effect, these nanostructures have shown fascinating physical properties, for instance, strong quantum confinement induced by the limited dimensions. These unique properties make them the best choice for the fabrication of quantum devices within nanometer scale.

As one of the most important nanostructures, nanowires (NWs) become an active research area within the nanoscience community. The growths of most of NWs were employing the vapor-liquid-solid (VLS) mechanism, introduced by Wagner and Ellis in the 1960's to grow Si submicrosize whiskers [2]. Although Si nanowires are useful, particular their easy incorporation with mature $\mathrm{Si}$ technology, which makes products cheap and suitable for mass production, the indirect band gap of $\mathrm{Si}$ is hindering the application of this material in many fields where direct band gap is essential, such as optoelectronics. In this regard, III-V compound semiconductors are dominating due to their direct band gap and flexibility in band gap and lattice engineering. In the early 1990s, Scientists from Hitachi employed the VLS approach to grow III-V nanowires $[3,4]$. At that time, good position and orientation control was achieved as well as the demonstration of the first $p-n$ junctions based on heterostructured nanowires [5]. These novel one-dimensional (1D) III-V nanostructures provide a good model system for investigating the dependence of electronic transport, optical, and mechanical properties on their confinement effects. They demonstrated the potential of these nanowires in the next-generation integrated circuits and functional devices, such as field-effect transistors [6-8], single-electron transistors [9], light-emitting devices [10], chemical sensors [11-14], and THz detectors [15].

The control of NW growth is considered to be one of the key points and the foundation of successful NW-based device fabrication. The benefit of NWs is that due to their small radial dimension and the large aspect ratio, the strain in axial heterostructures induced by the lattice mismatch can be relaxed within a small region close to the interface. Therefore, there is virtually no limitation in the choice of materials by the requirement of lattice-matching.

Another benefit of III-V semiconductor NWs is that by carefully choosing substrates, the growth of NWs can be self-assembled, and as-grown epitaxial NWs are free-standing. Additionally, by employing nanoscale lithography techniques, e.g., electron beam lithography, it is possible to realize the growth of NWs on pre-patterned substrates [16-20].

\footnotetext{
${ }^{1}$ Institute of Microstructure and Property of Advanced Materials, Beijing University of Technology, Beijing 100124, China

${ }^{2}$ School of Mechanical and Mining Engineering and Centre for Microscopy and Microanalysis, University of Queensland, St Lucia, QLD 4072, Australia * Corresponding author (email: ang.li@bjut.edu.cn)
} 
This opens a pathway to fabricate electronic devices with wrap-all-around gate electrodes based on aligned NWs $[9,21,22]$. The cylindrical geometry simplifies gate positioning at the tunnel junction, and the possibility to combine lattice-mismatched materials gives a great flexibility in the design of the tunnel junction [23].

In this review, recent progress on the growth of III-V semiconductor NWs and their heterostructures are reviewed: first, we deliver a general discussion on the crystal structure and the growth mechanism of III-V homo- or heterostructured NWs. Then the growth of different heteostructured III-V semiconductor NWs is reviewed. We focus on the morphology dependence, temperature influence, and III/V flux ratio dependent growth. The other main subject of this review is aimed at presenting advanced characterization technique of III-V heterostructured NWs by means of advanced electron microscopy, electron diffraction, Raman spectroscopy, and atom probe tomography. This review is composed of the following six sections:

- Section II: Crystal structure and Morphology of III-V nanowires

- Section III: Growth mechanism of III-V nanowires

- Section IV: Growth technique of III-V nanowires

- Section V: Sample preparation and advanced characterization techniques

- Section VI: Growth of III-V nanowire hetrostructures

- Section VII: Summary and outlook.

\section{SECTION II: CRYSTAL STRUCTURE AND MORPHOLOGY OF III-V NANOWIRES}

\section{Crystal structures of III-V NWs and their crystal defects found in III-V NWs}

In materials science, it is well known that the fundamental physical properties of materials are related to their structures. In this case, due to the fact that most III-V semiconductor NWs have different crystal structures with respect to their bulk phases, we expect that their basic physical properties will be substantially modified. The crystal structures of III-V NWs also attracted a lot of interest. It is well known that for most III-V binary semiconductors, the zinc blende (ZB) structure is the thermodynamically stable bulk crystal form. However, the most surprising feature of III-V NWs is that they often adopt the hexagonal wurtzite (WZ) structure in contrast to their bulk counterparts [24-28]. In the following we will discuss these two crystal structures and then discuss the factors that control the growth of these structures.

Typically, group-III and group-V atomic species are the ones building up the atomic layer which constitutes the NW crystal. The reason for the presence of different polytypes in NWs is that even though the bilayers have the same in-plane structure with respect to the most common $\langle 111\rangle$ B growth direction, the stacking sequence along the growth direction differs, and it often changes during growth. If each of the letters $\mathrm{A}, \mathrm{B}$, and $\mathrm{C}$ corresponds to an atomic layer, then the stacking is $\mathrm{ABCABC}$... for the $\mathrm{ZB}$ structure and $\mathrm{ABAB}$... for the most common hexagonal WZ structure, as shown in the upper panels of Figs 1a and b. The unit cells of the ZB and WZ structure are shown in lower panels of Figs $1 \mathrm{a}$ and $\mathrm{b}$, respectively. The group-III atoms are occupying $(0,0,0)$ positions and group- $\mathrm{V}$ atoms are taking up $(1 / 4,1 / 4,1 / 4)$ positions. On the contrary, in the unit cell of the WZ structure, the group-III atoms are in $(1 / 3,2 / 3,0)$ positions and group- $\mathrm{V}$ atoms are occupying $(1 / 3$, $2 / 3,3 / 8)$ positions. Thanks to the high resolution scanning transmission electron microscopy (STEM) with Cs-corrector, nowadays the polytypes of the III-V NWs can be easily resolved by high angle annular dark field (HAADF) image. As examples of atomic resolution HAADF images shown in Figs $1 \mathrm{a}$ and $\mathrm{b}$, by tilting the crystal to [110] zone axis, these two different polytypes can be distinguished clearly.

Due to the fact that the growth of NWs can be treated as a continuous stacking of bilayers along one direction, there are certain possibilities that the new bilayer may be faultily stacked. These faultily stacked bilayers will introduce planar defects in NW crystals. In a NW with ZB structure, twin planes can happen, reverse the stacking by forming ABCACBA... instead of ABCABC... and rotate the crystal by $60^{\circ}$ around the $\langle 111\rangle$ growth direction. In this case, the bilayer $C$ is the faultily stacked bilayer which creates the twin plane. However, twin planes are not allowed in $\mathrm{WZ}$ structure but stacking faults commonly happened by misplacing one single bilayer. When it happens, an ABACACA... will form instead of $A B A B A B$... It is interesting to notice that now there is a $\{B A C\}$ stacking due to the faultily stacked bilayer $\mathrm{C}$, and indeed, it is a small $\mathrm{ZB}$ segment embedded in WZ structure. In fact, the optical and electrical properties of III-V NWs are strongly related to the type of these crystallographic defects and their density [29], as one of the most attracting topics in the field. More efforts have been undertaken to control [30] the polytypic nature of $\mathrm{Au}$-assisted III-V NWs. As pioneer works, InP [31,32] and $\mathrm{GaP}$ [33] twin supper lattice structures were first reported (Fig. 2). For a long time, experimentalists have shown that by changing the growth parameters, such as NW diameter [34,35], growth temperature [36], or precursor flow [37] employed during the growth, it is possible to modify the stacking order of the bilayers. Consequently, the growth direction [38] or the crystal structure [34,36,37] of the NWs 

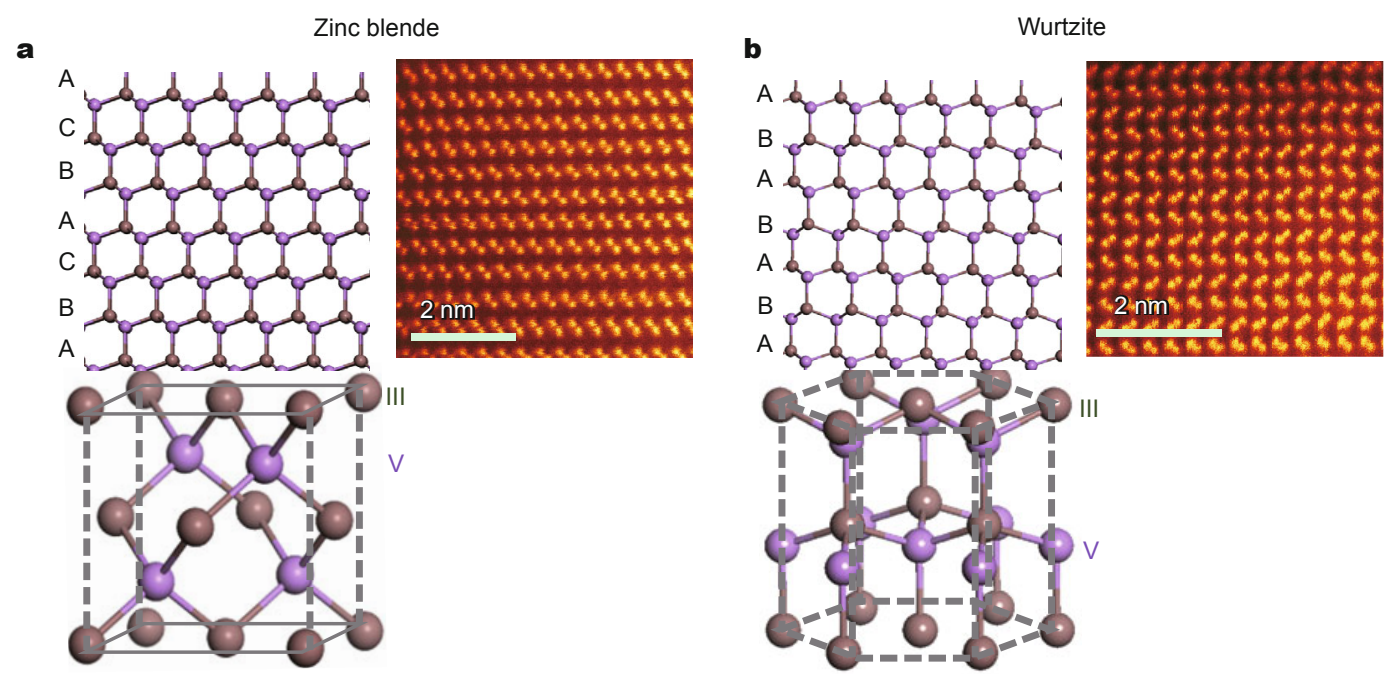

Figure 1 ( $\mathrm{a}$ and $\mathrm{b})$ The $\mathrm{ABC}(\mathrm{AB})$ stacking for the $\mathrm{ZB}(\mathrm{WZ})$ structures, their unit cells, and their typical atomic resolution HAADF STEM image.

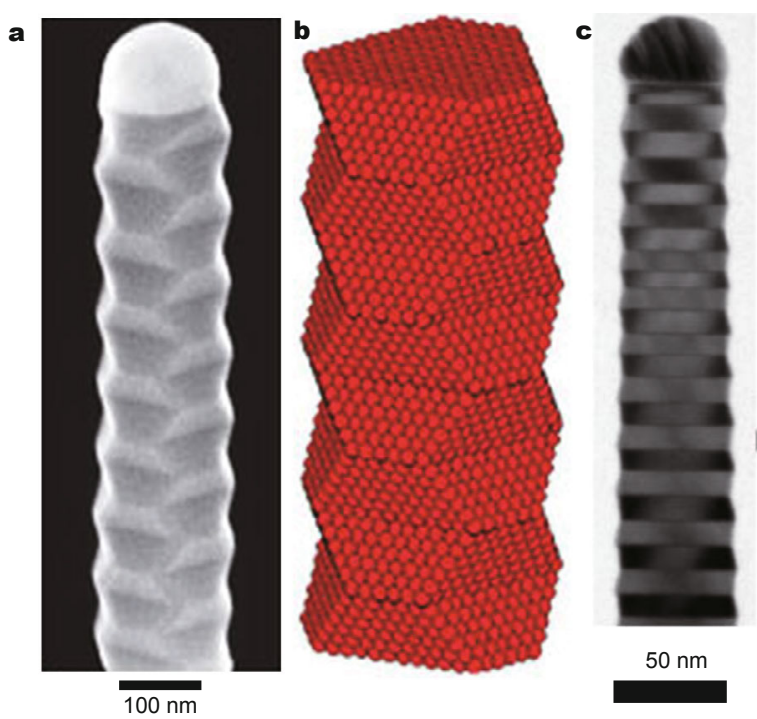

Figure 2 (a) SEM image of InP NW contains twinning super lattices (TSLs) and their atomic models (b). Reprinted with permission from Ref. [32] (Copyright 2009, Nature Publishing Group). (c) GaP NW contains TSL. Reprinted with permission from Ref. [33] (Copyright 2011, American Chemical Society).

can be altered.

However, the understanding of the mechanism behind these parameters are very limited. Very recently, Gil et al. [35] reported the growth of pure ZB phase GaAs NWs with diameter between 5 and $15 \mathrm{~nm}$, as shown in Fig. 3a. Interestingly, previous work predicted that within this diameter range only pure $\mathrm{WZ}$ phase should form regardless of the growth conditions $[39,40]$. Based on their experimental results, a plausible theoretical model is proposed in which the formation of either $\mathrm{WZ}$ or $\mathrm{ZB}$ phase NWs is not related to the diameter but the energy difference $(\Delta \mu)$ between two critical chemical potentials. The first one corresponds to the equality of the nucleation barriers for the critical $\mathrm{WZ}$ and $\mathrm{ZB}$ nuclei at the three phase line $\left(\Delta \mu_{\mathrm{TPL}}\right)$ and the second to the equality of the nucleation barrier for the $\mathrm{WZ}$ nucleus at the TPL and the ZB nucleus in the center of the liquid-solid interface under the droplet $\left(\Delta \mu_{c}\right)$. As it demonstrated in Fig. 3b, the red lines show the calculated $\Delta \mu$ NWs. According to these $\Delta \mu$, it can be seen that the GaAs $\mathrm{NW}$ phase could enter the WZ or ZB region by changing $\mathrm{Ga}$ content in the catalyst droplet or varying the growth temperatures.

\section{Morphology of III-V heterostructured NWs}

During the elongation of NWs, the total free energy is dominated by surface free energy of the interface between the solid phase and the liquid phase. Therefore, in order to minimize the total free energy, continuous formation of certain lowest energy planes are energetic favored. This gives the preferential growth orientation of the $1 \mathrm{D}$ crystals. In the case of NWs having ZB structures, e.g., low-temperature-grown GaP, InSb, GaSb, the new (111) planes with lowest energy are often formed at the solid-liquid interface, and therefore, ZB NWs tend to grow along the $\langle 111\rangle$ direction. It is worth noting that depending on whether surface is terminated by group III or V atoms, (111) planes for III-V compound semiconductors can be further distinguished into higher energy (111) A planes (group III terminated) and lower energy (111) B planes (group V terminated) [41]. Therefore, ZB NWs are generally observed to grow in $\langle 111\rangle \mathrm{B}$ direction [11]. On the other hand, NWs with WZ structure (e.g., high-temperature-grown GaP, InAs, AlAs) 

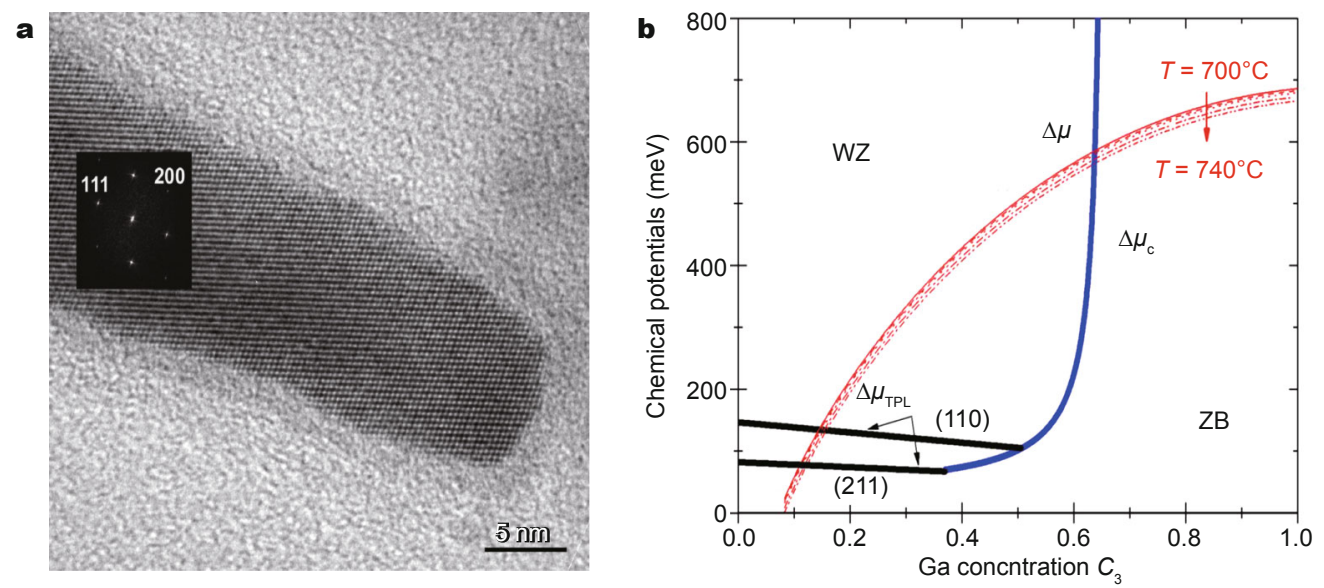

Figure 3 (a) HRTEM images taken along the [011] zone axis of GaAs NWs grown by Au-assisted at $715^{\circ} \mathrm{C}$ with diameter of 5-6 nm. Indexed fast Fourier transform (FFT) is shown in the inset. (b) Chemical potentials of GaAs in a liquid Au-Ga-As droplet during Au-catalyzed growth of GaAs NWs at temperatures between 700 and $740^{\circ} \mathrm{C}$ (red lines) as functions of the Ga concentration, compared to the critical chemical potentials $\Delta \mu \mathrm{TpL}$ and $\Delta \mu_{\mathrm{c}}$ for the WZ phase formation. Reprinted with permission from Ref. [35] (Copyright 2014, American Chemical Society).

are often observed to grow along the $\langle 0001\rangle$ direction and with $\{1100\}$ or $\{1120\}$ sidewall facets [42]. Furthermore, by employing different orientated substrate, catalyst, dimension of the catalyst, and the filling of group III elements in the catalyst droplet, the growth direction of the NWs can be altered into other low-index directions such as $\langle 001\rangle$, $\langle 110\rangle$ and $\langle 112\rangle[38,43-45]$.

Generally, two families of heterostructured NWs can be achieved by changing the growth parameters: core-shell (radial) and axial heterostructured NWs. Core-shell NWs can be realized when the surface diffusion length of the adatoms is strongly reduced, for example, by decreasing the growth temperature or growing with a high group- $\mathrm{V}$ flux under the ultra-high vacuum (UHV) growth condition. In this condition, not enough material is feeding the alloy droplet, and the supersaturation is not reached. At the same time, nucleation of new layers on the NW side facets becomes possible, and the growth switches to conventional two dimensional (2D) film growth on the sidewalls of the NWs, as shown in Fig. 4a. However, in the vapor phase epitaxy systems, e.g., metalorganic vapor phase epitaxy (MOVPE), since the diffusion length of the adatoms is much shorter than in the UHV systems, the shell growth is often realized by increasing the growth temperature in order to promote vapor-solid deposition on the NW sidewalls. In the case of core-shell heterostructured NWs, the lattice mismatch of the core material with respect to the shell plays an important role. In the case of lattice matched materials, e.g., the AlAs-GaAs system, commonly thin shell is epitaxially grown on the sidewall of the core without strain or dislocation. On the contrary, if there is an exis- tence of lattice mismatch, the strain induced defects will present in the shell structure. If the gas phase species reaching the NW are changed but the elongation of the NWs maintains during growth, axial heterostructured NWs will form. However, in this case, even if the NW continues growing axially, the chemical composition of the catalyst droplet changes and its chemical potential is modified. This may lead to a changing of the contact angle as well as the dimension of the catalyst particle and eventually change the dimension of the as-grown NW (as shown in Fig. 4c). InAs/InSb axial heterostructured NWs are an example of

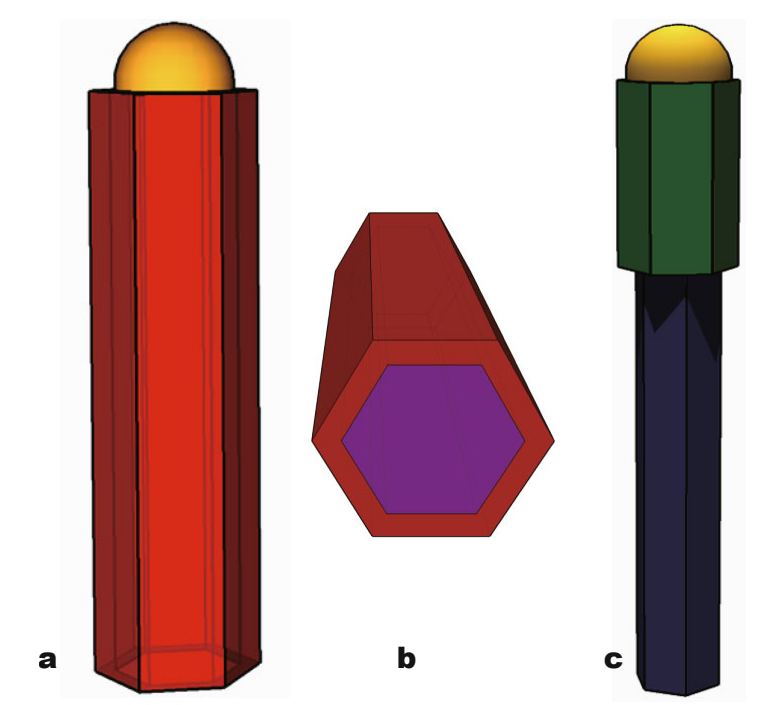

Figure 4 Different families of heterostructured NWs can be grown by Au-assisted epitaxial growth: (a) core-shell heterostructured NW and (b) its cross-section; (c) axial heterostructured NW. Different color indicates different material. 
this effect [46-48].

Due to the complexity of reactions taking place during the growth of heterostructured NWs, it is not easy to maintain only axial or radial growth. Therefore, tapering and lateral growth of NWs are commonly observed [49]. Different methods are reported to reduce the tapering of the NWs or suppress the lateral growth completely, such as several temperature steps, $\mathrm{HCl}$ in situ etching [50-53].

\section{SECTION III: GROWTH MECHANISM OF III-V NANOWIRES}

Most of the experiments discussed within this review are catalyst $(\mathrm{Au})$-assisted grown III-V heterostructured NWs. One fundamental aspect on the catalyst-assisted NW growth is the understanding of the growth mechanisms behind it. Nowadays, the VLS mechanism is widely accepted for the explanation of NW formation [54].

Back in the 1960s, by growing silicon microwires from the vapor phase, Wagner and Ellis first proposed the VLS mechanism to describe the growth of $1 \mathrm{D}$ whiskers [2]. This mechanism has been subsequently extended to nanometer scale and aims at explaining the growth of NWs [2,54,55]. In addition, recent in situ X-ray diffraction and thermal analysis carried out during passing $\mathrm{N}_{2}-\mathrm{NH}_{3}$ over Al-Ni alloy particles reveals an "extended VLS growth". In this case, the growth of AlN NWs is a physicochemical process dominated by the phase equilibrium of the Al-Ni alloy catalyst [56].

The conventional VLS model is based on the following experimental facts: first, the wire does not contain any screw dislocations [55] and second, a small metal particle is present on the top of the wire [2]. The scheme of this model is shown in Fig. 5: the metal particle becomes a liquid alloy with the feeding material at the growth temperature. In the first stage, the liquid alloy acts as a collector and the gas phase acts as a supplier. The gas phase molecules may decompose and are incorporated in the liquid alloy. This becomes supersaturated (i.e., it contains more feeding materials than the amount that can be dissolved), and the balance is recovered by the formation of a solid crystal at the liquid/solid interface, resulting in $1 \mathrm{D} \mathrm{NW}$ growth. The supersaturation is the most important driving force in the growth of NWs and will be discussed in detail later on.

In the case of catalyst-assisted growth of III-V NWs, the vapor phase of group-III and group-V precursors are the suppliers and the alloy droplets are the collectors [57]. The group- $\mathrm{V}$ materials have a short diffusion length on the surface [58], therefore, their contribution to the growth of NWs is mainly through the direct impingement on the catalyst droplets. In most of the cases the growth is usually carried out under group- $\mathrm{V}$ rich condition. Therefore, the group-V molecules adsorbing on the substrate surface only contribute to the planar 2D growth and seldom limit the axial growth of NWs. Specifically, it is known that As has a very low solubility in $\mathrm{Au}$ [59] and several post growth measurements confirm that no As is observed in the particles $[60,61]$. On the contrary, the group-III molecules usually have long diffusion lengths.

Concerning the growth of catalyst-assisted NW growth, Wacaser et al. [57] developed a preferential interface nucleation model. This model has completed the general VLS theory in the sense of explaining why the crystal prefers

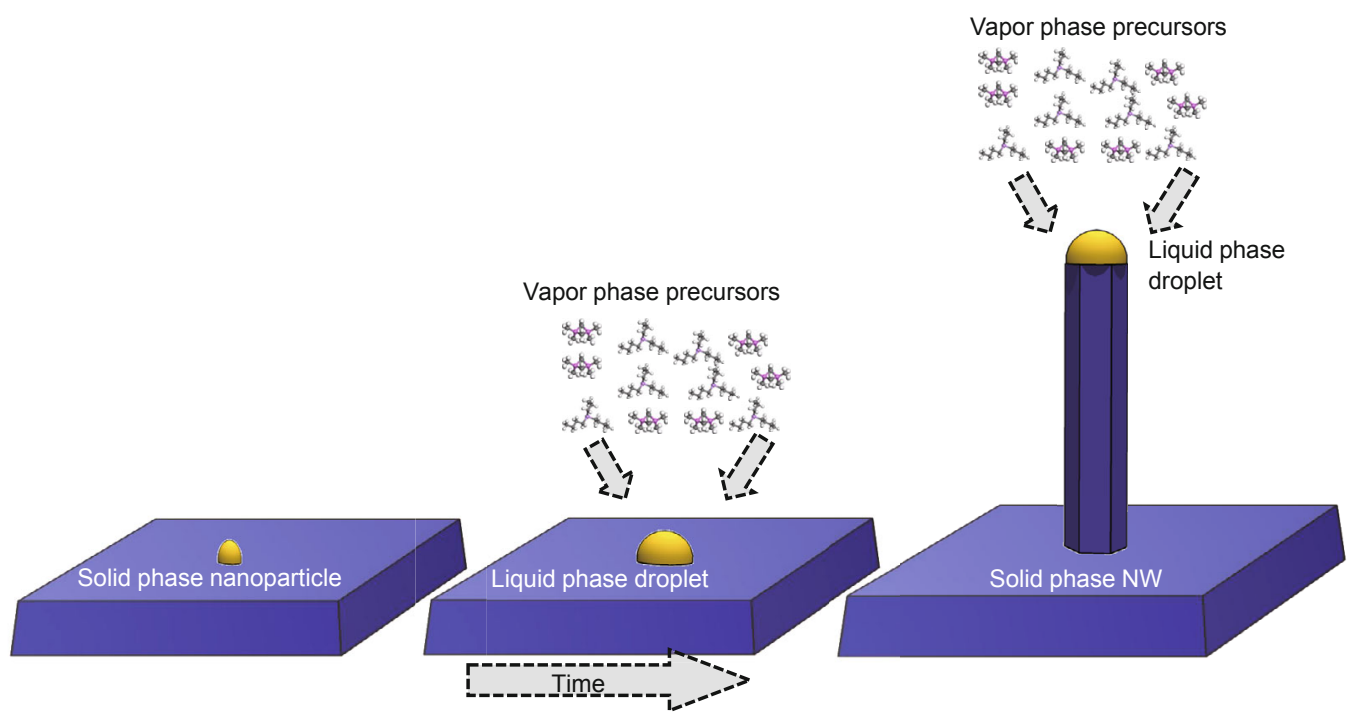

Figure 5 Scheme of the VLS growth mechanism. 
to grow along 1D instead of planar growth (2D). By calculating the different energy barriers for nuclei located in different sites, they demonstrated that the energy barrier has its minimum when nucleation happens at the three phase boundary (TPB), which is at the interface between vapor, liquid and solid phases. Therefore, the nucleation is more likely at the TPB than at the substrate, and this leads to the growth of NWs. Once nucleation takes place, a monolayer (ML) rapidly spreads to cover the whole liquid/ solid interface, forming one new layer of the NW, see Fig. 6. After these processes, another nucleation takes place at the TPB, and then a new ML is formed. As long as this process repeats, the crystal will grow in 1D fashion. Since each new layer along the growth direction is generated by a fast "birth and spread" growth $[24,62]$, this model also predicts a sharp interface formation in heterostructured NWs.

The growth rate of NWs is an easy-to determine quantity (i.e., from post-growth microscopy analysis), but is able to convey a wealth of information on the basic growth mechanisms of the NWs, and it is included in a suitable theoretical framework. In order to understand the general growth process, the conventional VLS model is demon- strated in Fig. 7, which includes three major transport pathways of the group-III materials: the surface diffusion $J_{\text {diff }}$, the direct impingement $J_{\text {dir }}$, and the desorption flux $J_{\text {des }}$. In the following we will first discuss the contribution of $J_{\text {diff }}$ to the growth process of NWs, which is strongly related to the growth kinetic mechanism. This mechanism takes into account that the adsorbed adatoms diffuse over the surface (substrate surface and NW sidewalls) and incorporate into the NW at an energetically favorable position, for example, alloyed with the catalyst droplet or at the step edges. Classically, the diffusion length $(\lambda)$ at a surface is derived as following.

As schematically shown in Fig. 8a, the overall process of the growth includes the following separate processes: (i) adsorption of atoms from the vapor phase, (ii) surface diffusion of the adatoms towards the growth front, and (iii) incorporation of the adatoms. If diffusion lengths are large with respect to the alloy particle size, we can neglect the adatoms impinging directly onto the catalyst particle and concentrate only on the dynamics of the species diffusing on the surface. Therefore, under conditions of equilibrium of the crystal with its vapor phase, the flux of the adsorbed
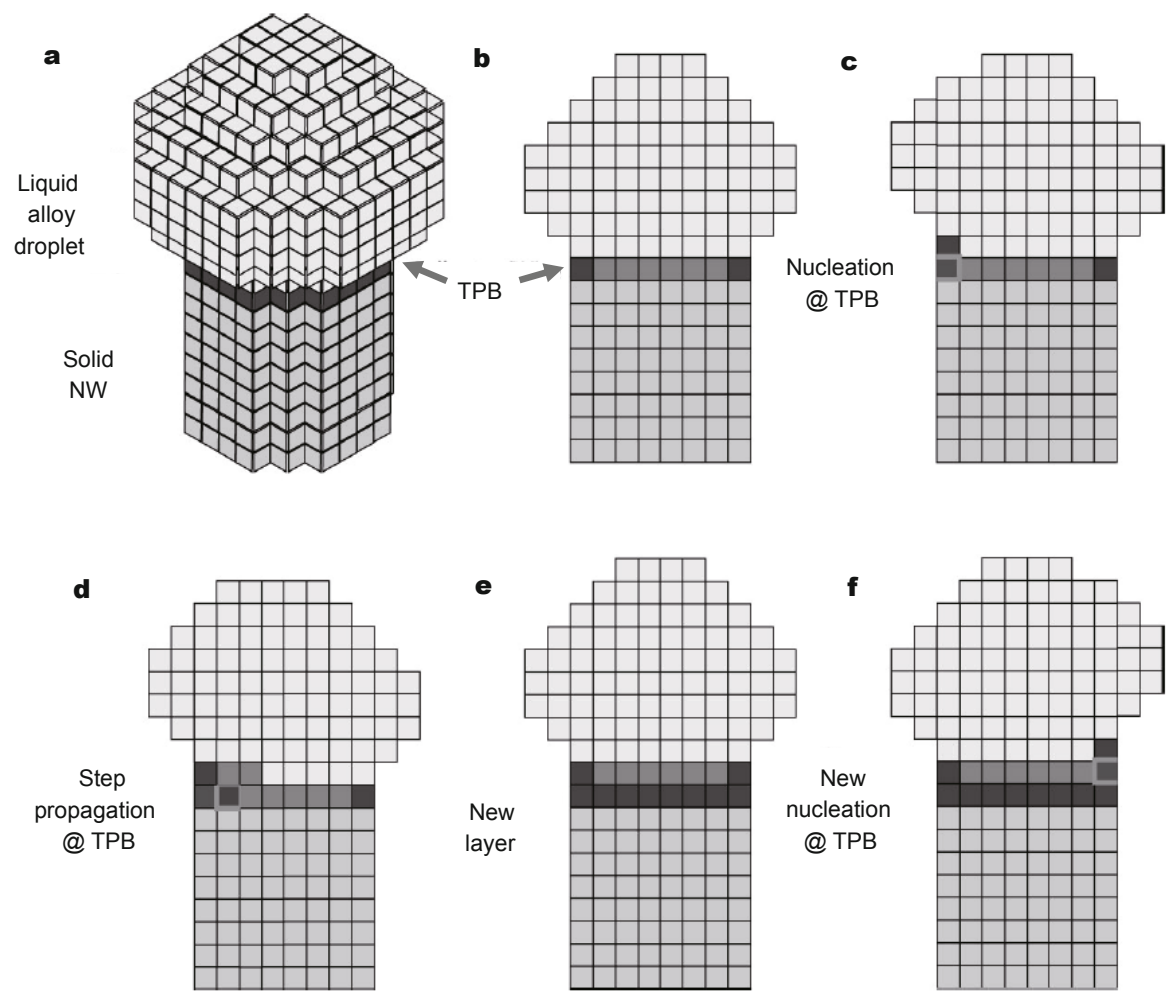

Figure 6 Illustration of preferential interface nucleation, birth, and spread growth of a NW. (a) 3D depiction of a NW illustrating the TPB as a dark line on the circumference of the droplet/NW interface. (b) Cross section of the wire depicted in (a). (c) Nucleation of a new layer at the TPB with the TPB being displaced in the growth direction. (d) Step propagation at the droplet/NW interface. (e) A new layer is completely formed. (f) Nucleation at a different site. Reprinted with permission from Ref. [57] (Copyright 2009, Wily-VCH Publishers, Inc.). 


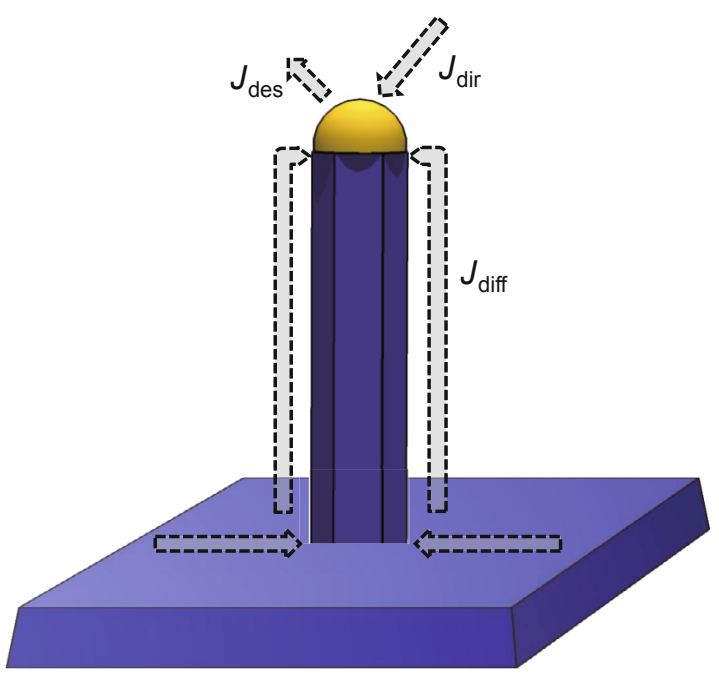

Figure 7 Schematic view of the three major material transport pathways which are usually considered in conventional VLS model.

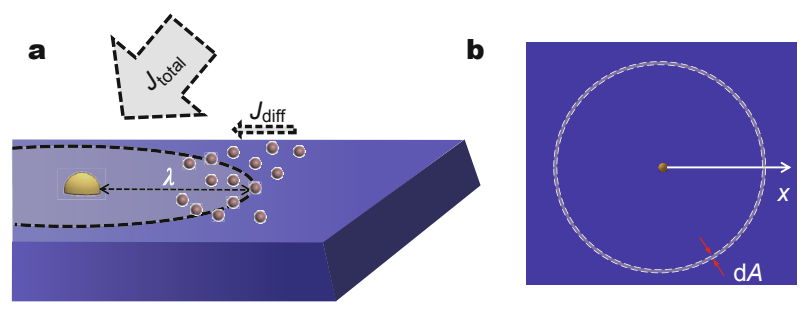

Figure 8 Schematic view of an individual Au catalyst droplet collecting adatoms through surface diffusion. In (a), $J_{\text {total }}$ is the flux of atoms from the vapor phase towards the substrate surface, $J_{\text {diff }}$ is the flux of adatoms diffusing towards the catalyst and the $\lambda$ is the mean distance covered by the adatoms during their life time $\tau$ on the surface. (b) Scheme of the collection area centered at the one individual NW.

atoms $P_{\infty} / \sqrt{2 \pi m k_{\mathrm{B}} T}$ equals the flux of desorbed atoms $n / \tau$. So that the adatom concentration $n$ has an equilibrium value $n_{\mathrm{e}}[60]$ :

$$
n_{\mathrm{e}}=\frac{P_{\infty}}{\sqrt{2 \pi m k_{\mathrm{B}} T}} \tau
$$

where $P_{\infty}$ is the equilibrium vapor pressure of infinitely large crystal, $m$ is the mass of the atoms and $\tau$ is the mean time of residence of the adatoms on the crystal surface before being re-evaporated and $\tau$ is given by:

$$
\tau=\frac{1}{v_{\perp}} e^{\left(\frac{E_{\mathrm{des}}}{k_{\mathrm{B}} T}\right)},
$$

in which $v_{\perp}$ is the vibrational frequency of the adatoms normal to the surface and $E_{\mathrm{des}}$ is the activation energy for desorption of an adatom from the crystal surface.
The mean distance that the adatoms can cover during their lifetime on the surface is:

$$
\lambda=\sqrt{D} \tau,
$$

where $D$ is the surface diffusion coefficient:

$$
D=a^{2} v_{\|} e^{\left(-\frac{E_{\mathrm{sd}}}{k_{\mathrm{B}} T}\right)}=a^{2} v_{\text {hop }} \text {. }
$$

Here $E_{\mathrm{sd}}$ is the activation energy for surface diffusion, $v_{\|}$is the vibrational frequency of the adatoms parallel to the crystal surface and $a$ is the mean distance between the adsorption sites. The rate of the adatoms hopping from one surface site to a neighboring one is $v_{\text {hop }}$. If we assume that $v_{\perp}=v_{\|}=v$, then

$$
\lambda=a \cdot e^{\left(\frac{E_{\mathrm{des}}-E_{\mathrm{sd}}}{2 k_{\mathrm{B}} T}\right)} .
$$

It is worth to note when the desorption energy $E_{\text {des }}$ is greater than the diffusion energy barrier $E_{\mathrm{sd}}$, we have $\lambda \gg a$ [60]. This allows the adatoms to diffuse from the adsorption site to the front of a NW and eventually to incorporate into the NW lattice.

The diffusion model is also used to describe the NW density dependence of the growth. A constant adatom concentration and no interaction between the adatoms lead to a random diffusion process. By neglecting the adatoms which are not diffusing to the catalyst, we can first simplify to a one dimension coordinate system as shown in Fig. 8b. Once the adatom is adsorbed at position $x_{0}$, after an average time $t_{\text {hop }}=1 / v_{\text {hop }}$ the adatom will move a distance $a$ in random direction. After $n$ steps the probability of finding the adatom at position $x$ is given by a Gaussian distribution:

$$
P(x)=\frac{1}{\sqrt{2 \pi} \sigma} e^{-\frac{\left(x-x_{0}\right)^{2}}{2 \sigma^{2}}},
$$

with $\sigma=a \sqrt{n}$ as the standard deviation [63]. From Equations ( 3 and 4 ), and $n=\tau v$, we can deduce:

$$
\sqrt{n}=\frac{\lambda}{a} \text {. }
$$

Therefore, the diffusion length equals the width of the distribution, and the probability reads:

$$
P(x)=\frac{1}{\sqrt{2 \pi} \lambda} e^{-\frac{\left(x-x_{0}\right)^{2}}{2 \lambda^{2}}} .
$$

Following Equation (8), the probability of adatoms reaching the NW via diffusion along the substrate surface 
follows a Gaussian distribution and centered at the NW [64]. The available material at a distance $r$ from the NW is proportional to the area of an infinitesimally thin circle: $\mathrm{d} A=2 \pi r \mathrm{~d} r$. Multiplying $P(x)$ with $\mathrm{d} A$ yields the amount of material $M$, which is collected from the NW base per unit time. Thus, the amount of material $M(r)$ reaching the NW from a distance $r$ in the unit time is:

$$
M \propto P(x) \cdot \mathrm{d} A=\frac{1}{\sqrt{2 \pi} \lambda} e^{-\frac{r^{2}}{2 \lambda^{2}}} \cdot 2 \pi r \cdot \mathrm{d} r .
$$

By integrating Equation (9) from the NW radius $\left(r_{\mathrm{w}}\right)$, to a half of the inter-wire distance from the NW $(R)$ the growth rate of NW results:

$$
\frac{\mathrm{d} L}{\mathrm{~d} t}=c_{1} \sqrt{2 \pi} \lambda\left(e^{-\frac{r_{\mathrm{w}}^{2}}{2 \lambda^{2}}}-e^{-\frac{R^{2}}{2 \lambda^{2}}}\right)+c_{2},
$$

where the constant $c_{1}$ relates to the material species and $c_{2}$ represents the growth rate associated only to the direct impingement on the catalyst droplet. According to Equation (10), the adsorbed incoming material will have a certain probability to diffuse toward the NW, which depends on $R$. This implies that when the surface diffusion length is longer than half of the inter-wire distance, the growth rate of NWs will be strongly influenced by the inter-wire distance, i.e., by the density of the NWs (see the scheme of Fig. 9). In fact, Jensen et al. [17] observed that the NW growth rate is strongly dependent on the density. They suggested that the

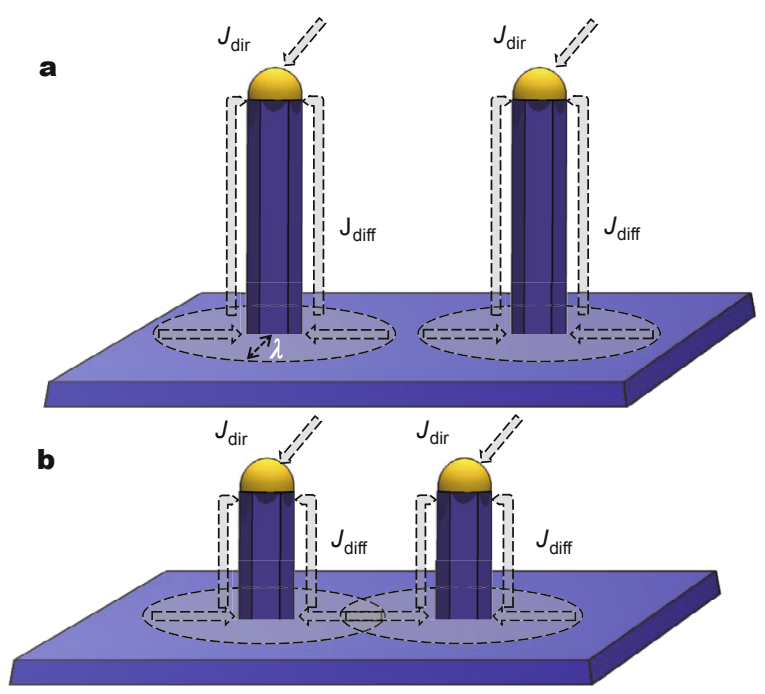

Figure 9 Scheme of the density dependence of NW growth rate. In case (a) the distance between two NWs is larger than two times $\lambda$, NWs can be treated as individual NW without competition. In case (b), however, the inter-wire distance is smaller than $2 \lambda$, and the NWs grow slower than in case (a). observed growth rate decrease by increasing NWs density is due to the reduction of the effective material collection area of each NW, as in the dashed circles shown schematically in Fig. 9.

Furthermore, several groups have experimentally observed that the axial growth rate of NWs is related to the catalyst particle size. For instance, the growth rate can increase with increasing NW diameter [65], decrease with increasing NW diameter [66] or even reach a maximum at a certain diameter [67]. In order to clearly understand this behavior, it is necessary to establish a model which combines both the kinetic and the thermodynamic driving forces. The thermodynamic driving force in the growth of NWs is the supersaturation. The supersaturation is defined as the difference in chemical potential $\Delta \mu$ between adatoms in the vapor phase and in the catalyst (Au) particle [67]:

$$
\begin{aligned}
\Delta \mu & =k_{\mathrm{B}} T \ln \left(\frac{p}{p_{0}}\right) \\
& =k_{\mathrm{B}} T \ln \left(\frac{p}{p_{\infty}}\right)-\frac{2 \sigma \Omega_{\mathrm{Au}}}{r_{\mathrm{w}}} \\
& \equiv \Delta \mu_{\infty}-\frac{2 \sigma \Omega_{\mathrm{Au}}}{r_{\mathrm{w}}},
\end{aligned}
$$

where $p$ is the adatom supply pressure, $p_{0}$ is the equilibrium pressure of adatoms in the catalyst particle, $p_{\infty}$ is the equilibrium pressure in an infinitely large seed particle (vapor pressure of adatoms in bulk Au), $\sigma$ is the surface energy density of $\mathrm{Au}, \Omega_{\mathrm{Au}}$ is atomic volume of adatoms in $\mathrm{Au}, r_{\mathrm{w}}$ is the NW radius, and $\Delta \mu_{\infty}$ is supersaturation over an $\mathrm{Au}$ surface with infinite radius of curvature [67]. For example, in the case of Au-assisted InAs NW growth, if we assume that the Au particle has a face-centered cubic (fcc) crystal structure and In atoms incorporate at these crystal positions, we get $\Omega_{\mathrm{Au}}=0.017 \mathrm{~nm}^{3}$, and using the bulk value for pure $\mathrm{Au}, \sigma=1.2 \mathrm{~J} \mathrm{~m}^{-2}$, we get $2 \sigma \Omega_{\mathrm{Au}} / k_{\mathrm{B}} T=4.2 \mathrm{~nm}[67,68]$, and this value is so called "characteristic Gibbs-Thomson radius" which will be discussed later on. It is worth to note that smaller particles have much higher surface energy density than the larger ones. This leads to an increase of the term $2 \sigma \Omega_{\mathrm{Au}} / r_{\mathrm{w}}$ in Equation (11), so that the chemical potential of the catalyst particle can be varied by changing its dimension. The thermodynamic nature behind this is the so called "Gibbs-Thomson" (GT) effect, which consists in the increase of the chemical potential of the thermodynamic phase due to the presence of a surface. In the early stage of the understanding of the VLS mechanism, Givargizov and Chernov [69] used the Gibbs-Thomson effect as an interpretation of the diameter related growth rate, $d L$ / 
$\mathrm{d} t\left(r_{\mathrm{w}}\right)$, of Si NWs:

$$
\frac{\mathrm{d} L}{\mathrm{~d} t}\left(r_{\mathrm{w}}\right)=K\left(\Delta \mu_{\infty}-\frac{2 \sigma \Omega_{\mathrm{Au}}}{r_{\mathrm{w}}}\right)^{2} .
$$

Now we can consider the limit case when the growth rate becomes zero. Then the critical diameter of NW is given by:

$$
d_{\mathrm{c}}=2 r_{\mathrm{c}}=2 \cdot \frac{2 \sigma \Omega_{\mathrm{Au}}}{\Delta \mu_{\infty}}=\frac{4 \sigma \Omega_{\mathrm{Au}}}{k_{\mathrm{B}} T \ln \left(\frac{p}{p_{\infty}}\right)}
$$

This means that for NWs with diameter smaller than $d_{c}$, the growth will be suppressed. Moreover, according to Equation (13), the critical diameter will decrease by increasing the growth temperature which implies that NWs with small diameter can be grown by simply increasing the growth temperature [70].

Dubrovskii et al. [71,72] were the first to demonstrate the possibility to combine both the kinetic and the thermodynamic mechanisms within a general growth model. Within this model, the growth rate of the NW is given by:

$$
\begin{aligned}
\frac{\mathrm{d} L}{\mathrm{~d} t}= & V\left[1-e^{\left.\left(\frac{\Delta \mu_{\mathrm{LS}}-\Delta \mu_{\mathrm{AS}}}{k_{\mathrm{B}} T}+\frac{r_{\mathrm{GT}}}{r_{\mathrm{w}}}\right)\right]}\right. \\
& \times\left[\frac{1}{\sin ^{2}(\beta)} \cdot e^{\left(\frac{\Delta \mu_{\mathrm{AS}}-\Delta \mu_{\mathrm{VS}}}{k_{\mathrm{B}} T}\right)}+\frac{2 \lambda K_{1}\left(\frac{r_{\mathrm{w}}}{\lambda}\right)}{r_{w} K_{0}\left(\frac{r_{\mathrm{w}}}{\lambda}\right)}\right],
\end{aligned}
$$

where $V$ is the $2 \mathrm{D}$ deposition rate $(\mathrm{ML} / \mathrm{s}), \Delta \mu_{\mathrm{LS}}$ is the difference of chemical potential between the particle and the substrate, $\Delta \mu_{\mathrm{AS}}$ is the difference of chemical potential between the adsorbed species which are able to diffuse toward the particle and the substrate. $\Delta \mu_{\mathrm{vs}}$ is the difference of chemical potential between the vapor and the substrate, $\beta$ is the particle contact angle, $K$ and $K_{0}$ are modified Bessel functions of the second kind. $r_{\mathrm{GT}}$ is the characteristic radius describing the GT effect in the droplet: $r_{\mathrm{GT}}=2 \sin (\beta)$ $\left(\sigma_{\mathrm{LV}} \Omega_{\mathrm{L}} / k_{\mathrm{B}} T\right)$, where $\sigma_{\mathrm{LV}}$ is the particle-vapor interface energy and $\Omega_{\mathrm{L}}$ is the elementary volume in the liquid. This model agrees well with experimental observations for the growth rate of GaAs NWs (see Fig. 10) [72], where only the contribution of surface diffusion is taken into account but the other material transport pathways such as direct impingement and desorption are neglected. The value of $r_{\mathrm{GT}}$ $=3.5 \mathrm{~nm}$, used in calculations, corresponds to a $30 \%$ alloy of liquid Ga with Au. The obtained diffusion length on the
GaAs (111)B surface $(\lambda)$ is about $95 \mathrm{~nm}$.

Most important, this diffusion induced growth model is a function with one maximum, determined by the competition of the adatom fluxes to the top droplet and the GT effect in the droplet. It predicts that the NW growth rate will reach to a maximum value for a certain diameter which is dependent on the growth conditions, techniques and materials. In the case of Fig. 10, it is marked by the dashed line and it is about $53 \mathrm{~nm}$. On the left side of the dashed line, e.g., for NWs with smaller diameter, the GT effect is limiting the growth rate. For larger diameters, the limiting factor is the amount of material reaching the catalyst through diffusion. As shown by Equation (10) the growth rate is a monotonous decreasing function of the diameter.

\section{SECTION IV: GROWTH TECHNIQUE OF III-V NANOWIRES}

The highly sophisticated growth techniques available in both research and industry are the reasons why semiconductors are widely used for the device fabrication. For decades, progress in crystal growth techniques such as metalorganic vapor phase epitaxy (MOVPE), molecular beam epitaxy (MBE), chemical beam epitaxy (CBE), has made the fabrication of semiconductor heterostructures and novel active devices possible. Recent progress in fabricating low-dimensional nanostructures takes advantage of alignment of atoms on the substrate to induce the self-organized structures, which is the main subject discussed in this section.

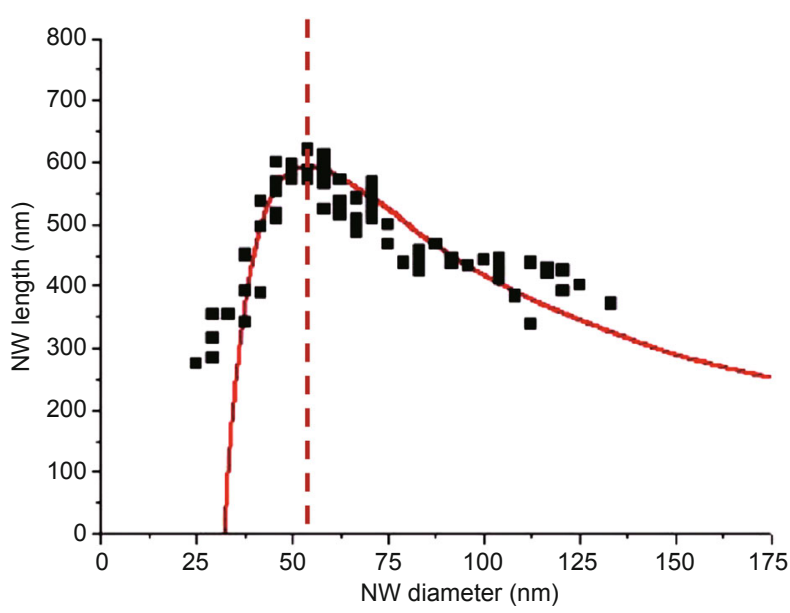

Figure 10 Experimental NW length vs. diameter dependence (black squares) of GaAs NWs and theoretical curve (red solid line) obtained by employing the Equation (14). Reprinted with permission from Ref. [72] (Copyright 2009, American Physical Society). 


\section{Metalorganic vapor phase epitaxy}

MOVPE, also known as metalorganic chemical vapor deposition (MOCVD) is a major process in the manufacturing of optoelectronics. As shown in Fig. 11a, MOVPE is a complex chemical vapor phase deposition method. There are normally different types of precursors in the MOVPE: the hydrides $\left(\mathrm{AsH}_{3}, \mathrm{PH}_{3}, \mathrm{Si}_{2} \mathrm{H}_{6}, \mathrm{GeH}_{4}\right.$, etc. $)$ and the metalorganics (trimethylgallium (TMGa), trimethylindium (TMIn), trimethylaluminium (TMAl), tert-dimethylaminoantimony (TDMASb), etc.). Hydrogen (nitrogen for nitride materials), is usually used as a carrier gas in order to transport the metalorganics precursors from the bubblers into the reactor. The amount of metalorganic va- por transported depends on the rate of carrier gas flow and the bubbler temperature.

The growth occurs in the reactor, which is a chamber made of a material that is resistant to the chemicals used and high temperatures. The substrate is placed on a heated susceptor in the reactor. After the precursors' molecules arrived on the substrate, they will be pyrolysed thermally and/or catalytically and leave the required atoms on the substrate surface. The atoms will diffuse on the substrate surface and eventually bond to the surface then a new crystalline layer is epitaxially grown. As can be seen in Figs $11 \mathrm{~b}$ and c, two categories of MOVPE reactors are using recently in order to perform the growth of semiconductor NWs:

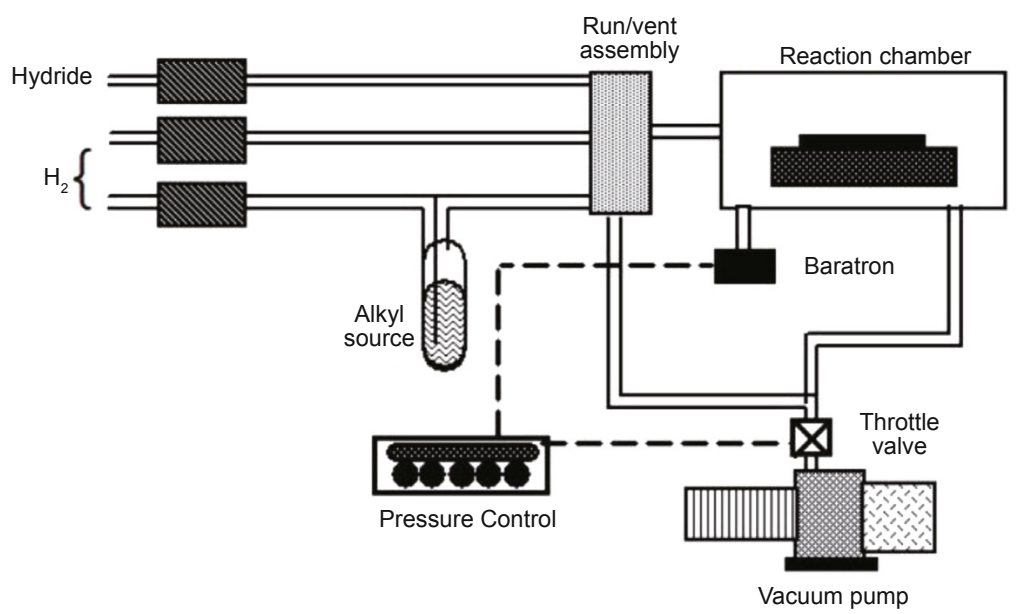

b

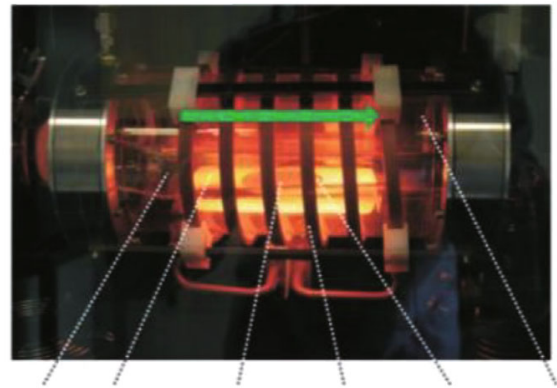

Precursor
flow Liner Susceptor Sample RF coil Satellite $\underset{\text { reactor wall }}{\text { Water cooled }}$

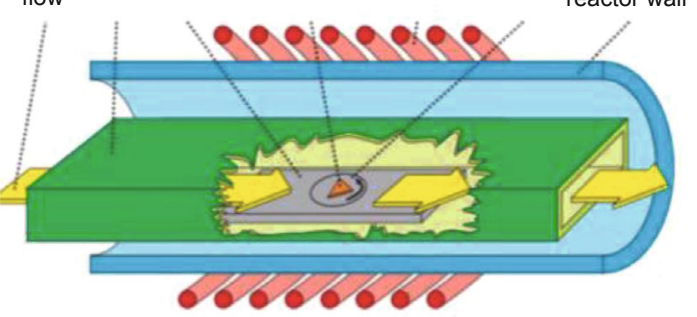

c
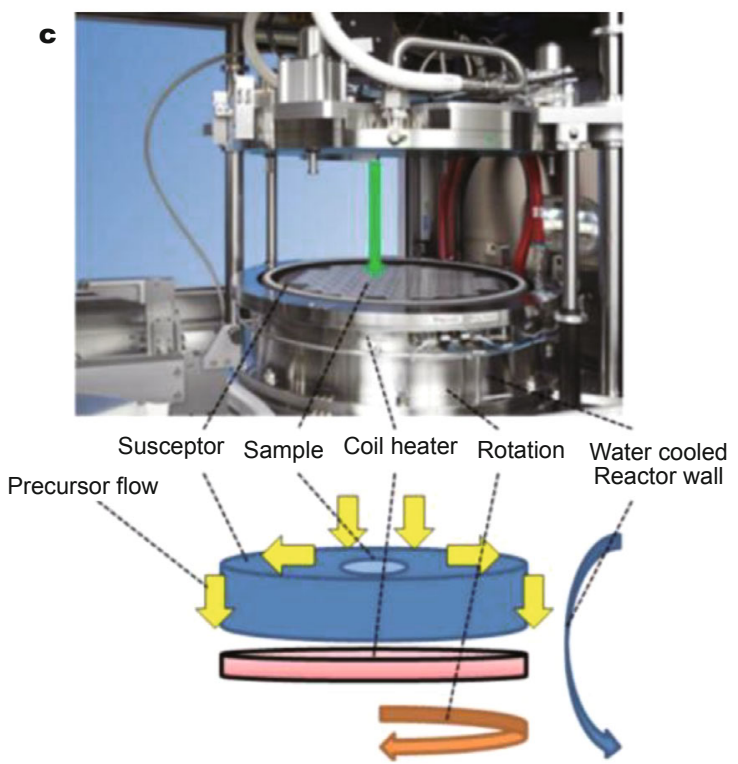

Figure 11 Schematic of MOCVD reactor components, and horizontal (a and b)/vertical (shower head) (c) MOPVE actual reactor and their schematic geometry. 
horizontal reactors and vertical (i.e., shower head) reactors. In a horizontal MOVPE, the precursors enter from one end of the liner and flow parallel to the substrate wafer. Depending on if the precursors need to be pre-cracked or not, hot-wall or cold-wall reactors can be chosen to process the growth. On the other hand, in the case of the shower head reactors, as shown in Fig. 11c, the gases are introduced into the react chamber perpendicularly over the entire substrate surface via the water-cooled shower. The distance between the shower head and the substrate is variable. And the substrate lie on a rotating susceptor which is heated by several separate resistive heaters in order to keep a uniform temperature over the entire surface. The recently developed shower head reactor is more advanced than the conventional horizontal reactor. Due to the fact that in the horizontal reactor, the precursors first meet the hot susceptor before the substrate, which introduces material deposition on the susceptor. The release of deposited material may cause more severe memory effects to the following growths. However, the shower head reactor does not have hot parts upstream, so the growth using shower head will suffer less from memory effects. Second, due to the small distance between the shower head and the substrate, fast gas switching is achieved. Furthermore, the gases are introduced into the reactor through separate openings in the shower head to achieve an even distribution of process gases, which introduces improved uniformity across the entire substrate. All these aspects make the shower head MOVPE a more promising candidate for heterostructure NW growth with sharp interface.

\section{Molecular/chemical beam epitaxy}

Both MBE and CBE are growth techniques carried out in UHV (i.e., lower than $10^{-9}$ Torr) chamber and with slow deposition rate which aim to produce high quality materials. The MBE system was first developed at the Bell Labs in the late 1960s [73], followed by the development of the CBE technique in the early 1880s [74-76]. The main difference between $\mathrm{MBE}$ and $\mathrm{CBE}$ is that, the $\mathrm{MBE}$ technique is using ultra-pure form solid which is heated in separate quasi-Knudsen effusion cells until they begin to slowly sublime. However, similar to MOCVD, the precursors used in the CBE system are metal organics (MO) but without carrier gas [76]. The use of only group-V precursors in CBE system, like tertiarybutylphosphine (TBP) and tertiarybutylarsine (TBAs), yields more stable beam fluxes with respect to MOCVD and a better control of the As/P ratio than MBE. Additionally, the use of MO sources of groupIII materials is motivated by their long-term stability and easy handling when changing sources. Furthermore, in the
CBE system as in MBE, the growth is always carried out inside an UHV environment. At such low pressures, the mean free path $l$ of a molecule is given by:

$$
l=\frac{k_{\mathrm{B}} T}{\sqrt{2} \pi d^{2} p},
$$

where $\kappa_{\mathrm{B}}$ is the Boltzmann constant, $T$ is the temperature, $p$ is pressure, and $d$ is the diameter of the gas particles. Thanks to the UHV environment, $l$ in a MBE/CBE system is of the order of $10^{5}$ meters facing to $\sim 5 \mu \mathrm{m}$ in MOVPE system. Therefore, the molecular transport occurs ballistically. Another advantage of the use of the UHV chamber is its compatibility with electron diffraction probes such as reflection high-energy electron diffraction (RHEED), which can provide fundamental in situ information on growth mechanism and crystal structure [77]. Such characteristics allow to carry out high-quality and uniformly epitaxial growth on wafers with large area.

One typical MBE/CBE setup (Riber Compact-21 III-V system) is schematicly shown in Fig. 12. A stainless-steel growth chamber is kept pumped with a turbo pump (Fig. 12b) and an ion pump (Fig. 12c). These two pumps provide a base pressure of $10^{-10} \mathrm{mbar}$. The turbo pump is kept pumping alone during the growth. A platen manipulator is capable of continuous rotation (up to $60 \mathrm{rpm}$ ) while heating up to more than $1000 \mathrm{~K}$. Additionally, the inside walls of the growth chamber are surrounded by a cryopanel which is cooled down to $77 \mathrm{~K}$ by liquid nitrogen (see Fig. 12), which allows to reduce the background pressure. Moreover, the cryopanel ensures that samples only see cold stainless-steel during the growth in order to avoid "memory effect" when switching materials. The growth chamber is usually connected to a preparation chamber, which is pumped by an ion pump. The substrates are degassed prior to the growth inside the preparation chamber to remove mainly the water from the substrate surface. Connected to the preparation chamber, there is a load-lock module pumped by a small turbo pump to transfer the sample from air into the system. All UHV components are able to resist a bake-out temperature of $150^{\circ} \mathrm{C}$ which is necessary to remove the water condensation from the walls and the cryopanels inside the growth chamber after closing the system.

In order to understand and optimize the interface within axial hetrostructures, Glass et al. summarized the growth temperature of VLS grown Au-assisted homostructures by MBE. The temperature ranges is shown in Fig. 13 in which the temperature window might slightly vary with the V/III ratio and with the NW diameter. Interestingly, the temperature window is quite similar for the structures with 


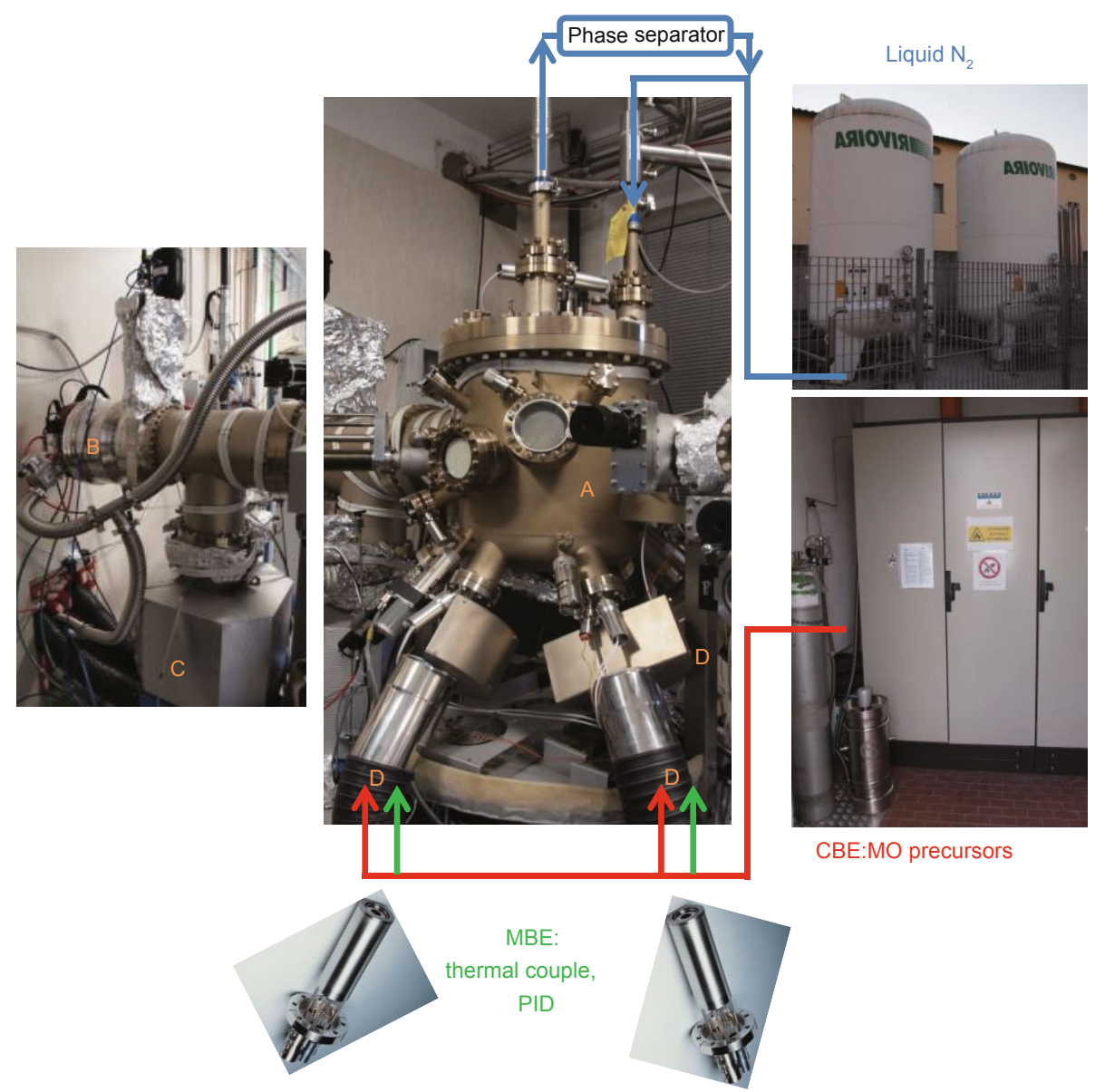

Figure 12 Picture of the Riber Compact $21 \mathrm{MBE} / \mathrm{CBE}$ system. A: growth chamber; B: turbo pump; C: ion pump; D: precursor injectors (red) of CBE or solid source effusion cell (green) of MBE; E: liquid nitrogen vessels, and F: gas cabinet of CBE.

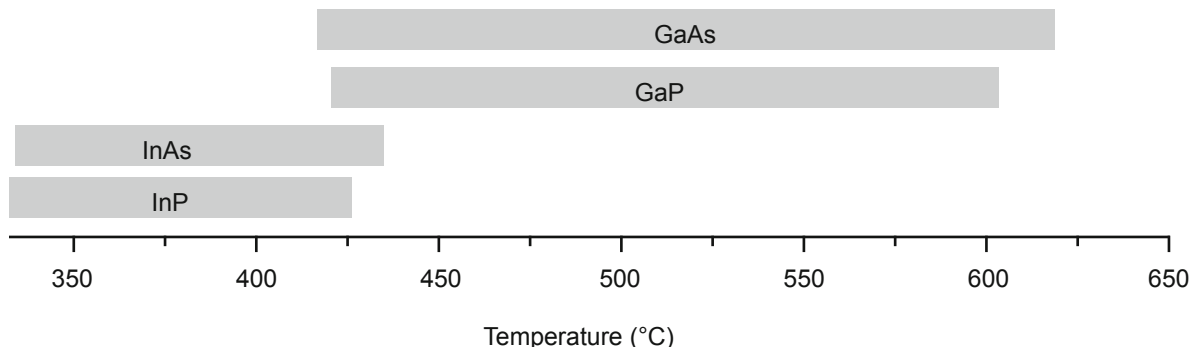

Figure 13 Ranges of substrate temperatures for which NWs of different binary III-V alloys can grow by Au catalyst-assisted MBE. Reprinted with permission from Ref. [78] (Copyright 2011, Bentham Science Publishers).

group-III atoms but the overlap is small for structures with different group-III atoms. This is solid evidence that the growth temperature for NW is mainly influenced by the chemical composition which is strongly associated with the supersaturation of III-Au alloy. In fact, compared with InAs/GaAs heterostructures, the NWs containing InAs/ InP $[79,80]$ and $\mathrm{GaP} / \mathrm{GaAs}[81,82]$ heterostructures show much sharper interfaces.

\section{SECTION V: SAMPLE PREPARATION AND ADVANCED CHARACTERIZATION TECHNIQUES}

\section{Sample preparation}

Before starting the NW growth, catalysts have to be deposited on the surface of the substrate. Usually the catalyst is a 
metal which can form a metal-III alloy droplet and plays a role as material collector during the NW growth. In most of the catalyst-assisted NW growth, the usually employed catalyst material is gold ( $\mathrm{Au})$. And there are three different widely applied approaches to deposit Au catalyst particles on the substrate surface, as schematically shown in Fig. 14. The first approach is to deposit commercial metal colloids on the substrate surface by drop casting a colloid solution on the substrate. The nanoparticles (NPs) will stick on the substrate by van der Waals forces, as the schematic in Fig. 14a shows.

However, several drawbacks have limited the application of this method. First of all, due to the van der Waals forces between the NPs, the Au NPs are mostly agglomerated instead of forming single particles on the substrate. Secondly, the impurities in the solution will eventually be left onto the substrate after deposition, preventing the growth of NWs. Other alternative approaches are thermal dewetting and lithography-defined patterning (Figs $14 \mathrm{~b}$ and c). In the following, we will first demonstrate the principles of thermal dewetting of Au films, then compare the growth result between thermal dewetting Au films and colloid deposition, in the end the lithography-defined patterns (selective area growth) will be present in detail.

\section{Sample preparation}

The simplest method for the formation of NPs on the sub- strate is through the dewetting process of a thin metal film. This process is driven by the reduction of the surface energy of the film and the interface energy between the film and the substrate. This process can be induced by thermal annealing. Dewetting proceeds by surface diffusion even in the solid state will below the melting temperature of the film [83-85]. In the case of ideal, defect-free, and homogeneous films, theoretical predictions claim that the surface energy driven mechanism starts at the film boundary, with edge agglomeration via capillary edge instability, and then followed by particle formation via Rayleigh instability $[86,87]$. However, in reality, thermal deposited metal films comprise defects and fluctuations in the film thickness as well as by edge agglomeration $[88,89]$. Therefore, voids can nucleate due to the film thickness fluctuations (spinodal dewetting), or at defects which are then followed by void growth and particle formation [87]. For polycrystalline metallic films, dewetting is also affected by the character of grain boundaries [89]. Concerning all effects above, dewetting of polycrystalline metallic films on a flat substrate usually leads to a broad distribution of particle size and spacing [90].

After deposition, a continuous thin film formed by Au grains is obtained as shown in Fig. 15a. In order to allow the NW growth, the substrate has to be pre-annealed inside the growth chamber with TBAs flow to form NPs and to remove the native oxide. Within this deoxidation procedure,

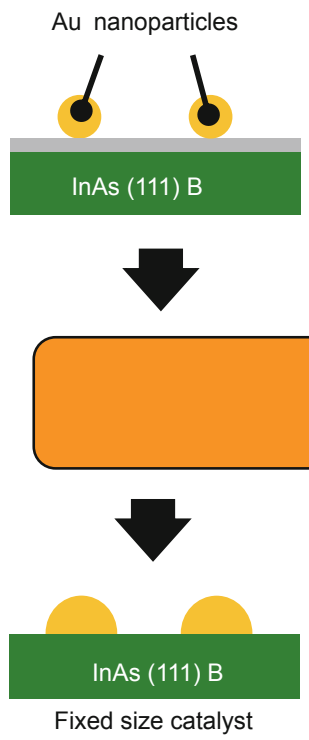

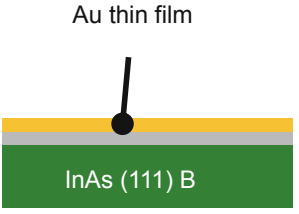

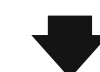

c

Lithographically defined

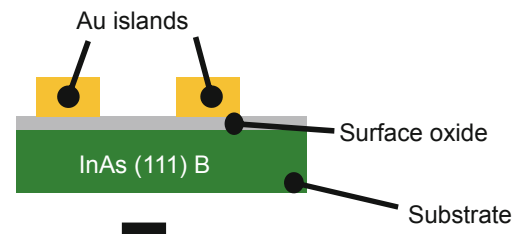

Heating

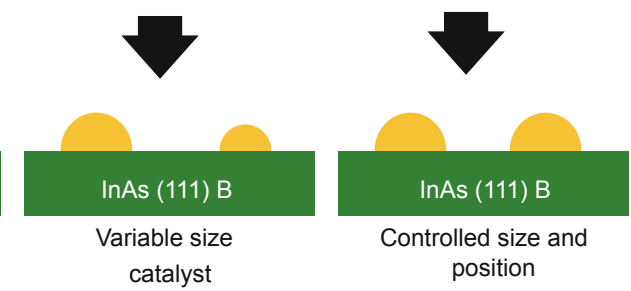

Figure 14 General approaches to deposit catalyst materials: (a) deposition of Au colloids; (b) thermal dewetting of Au thin film; (c) electron beam lithography (EBL)-defined Au pattern. 

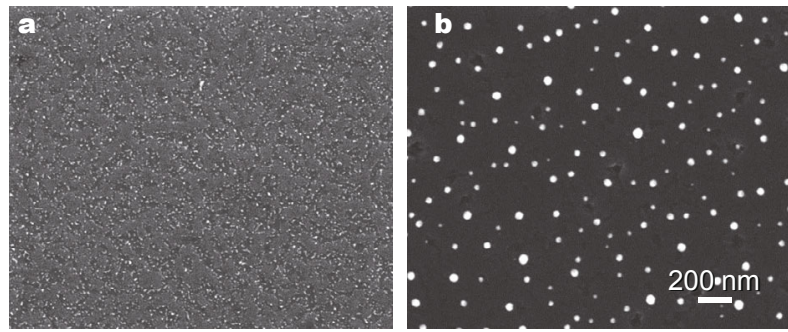

Figure 15 Plane-view SEM images of InAs substrate surface (a) after thermal deposition of a $0.5 \mathrm{~nm}$ thin $\mathrm{Au}$ film and (b) after the thermal dewetting process at $520^{\circ} \mathrm{C}$ for $20 \mathrm{~min}$.

the pre-deposited Au film will be dewetted and NPs will form. The deoxidation temperature is strongly dependent on the employed substrate. Since the dewetting temperature is higher than the eutectic point, the grains in the continuous Au film will form sparse Au-III droplets flowing on the substrate. From the SEM image of Fig. 15b, the alloy NPs are clearly visible. In order to acquire quantitative measurements of the alloy NPs formed by thermal dewetting, the area of the particles is measured from plane view SEM images and then converted into particle diameter by assuming that they have a circle shape.

The diameter distribution and density of the NPs will strongly influence the NW diameter and density. Therefore, it is important to control the thermal dewetting procedure. Previous studies $[90,91]$ on the dewetting process of thin metal films show that the key parameters which can tune the particle size and density after thermal dewetting are the film thickness $\left(h_{\mathrm{au}}\right)$, the dewetting temperature $\left(T_{\mathrm{ann}}\right)$, and the dewetting time $\left(t_{\mathrm{ann}}\right)$. In order to understand how these three parameters influence the NWs growth results after the dewetting process, Gomes et al. [92] conducted a systematic study by employing a homemade SEM image analysis software. In their study, $T_{\text {ann }}, h_{\text {au }}$ and $t_{\text {ann }}$ are varied individually while the growth of InAs NWs is using a same protocol. In Figs 16a-d, statistic of as-grown InAs NWs diameter after different dewetting method and different colloid diameters is shown. Interestingly, the detail statistic shown in Fig. 16e shows individual peaks corresponding to NWs seeded from single and agglomerated colloidal NPs. A comparison of the NW diameter distribution grown from Au thin film annealing and colloids, is shown as the plot of $\Delta d v s$. average diameter $\left(d_{\text {avg }}\right)$ as shown in Fig. $16 f$.
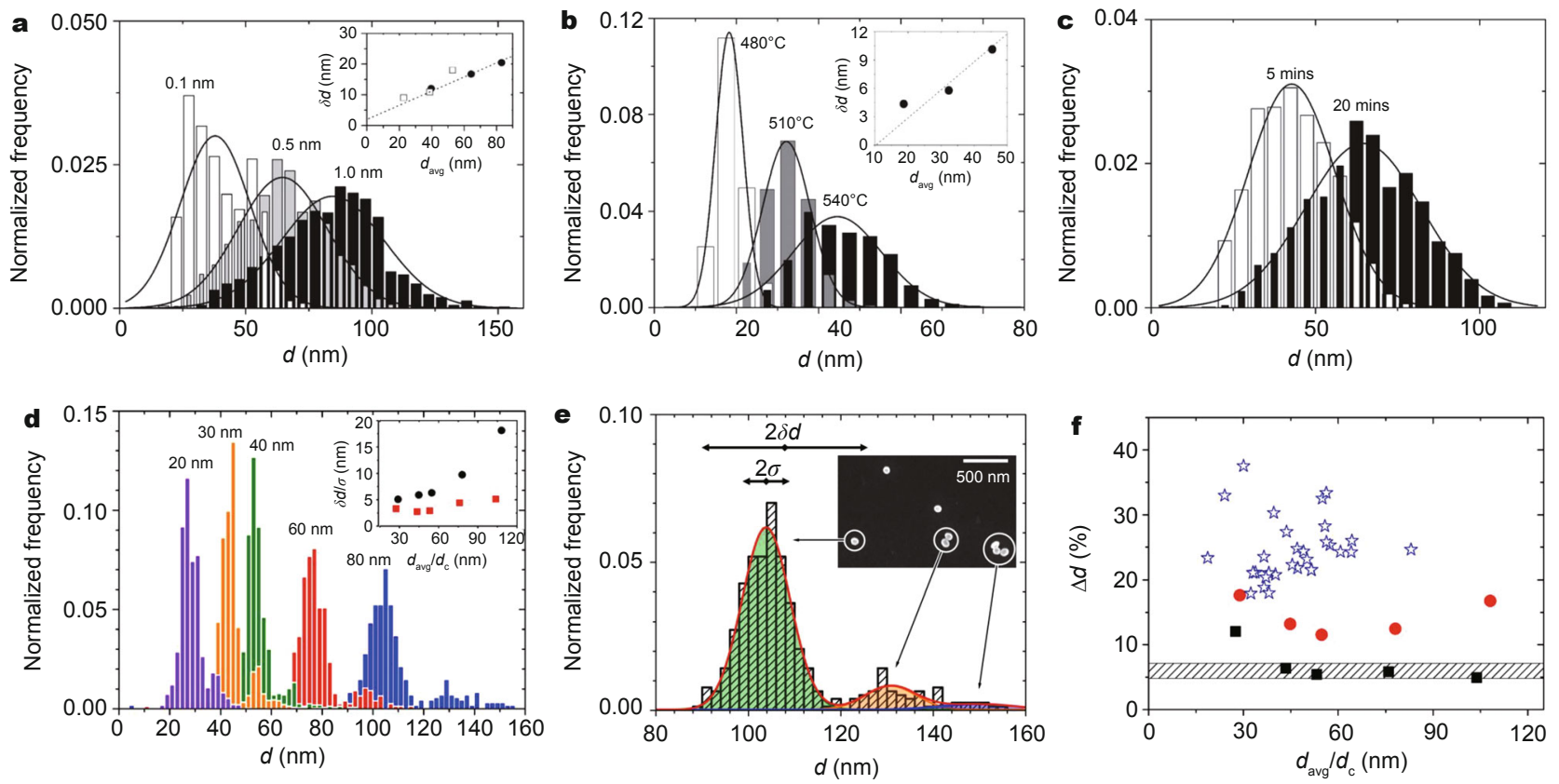

Figure 16 Experimental results of diameter $d$ distribution of InAs NWs with different dewetting Au film conditions and different colloid diameters. The superimposed lines are Gaussian fits of the data. (a) At fixed $T_{\text {ann }}=510^{\circ} \mathrm{C}$ and $t_{\text {ann }}=20$ min and different $h_{\text {au }}: 0.1$ (white), 0.5 (grey) and $1.0 \mathrm{~nm}$ (black). (b) At fixed $h_{\mathrm{au}}=0.1 \mathrm{~nm}$ and $t_{\mathrm{ann}}=5 \mathrm{~min}$ for different $T_{\mathrm{ann}}: 480^{\circ} \mathrm{C}$ (white), $510^{\circ} \mathrm{C}$ (grey) and $540^{\circ} \mathrm{C}$ (black). (c) Comparison of InAs NW diameter statistics at a fixed $h_{\text {au }}=0.5 \mathrm{~nm}$ and $T_{\text {ann }}=510^{\circ} \mathrm{C}$ and annealed with $t_{\text {ann }}=5$ and $20 \mathrm{~min}$. (d) From colloidal NPs of diameter 20,30, 40,60, and $80 \mathrm{~nm}$ at $T_{\text {ann }}=645^{\circ} \mathrm{C}$ and $t_{\text {ann }}=20 \mathrm{~min}$. (e) Diameter distribution of InAs NWs grown from $80 \mathrm{~nm}$ colloids at $T_{\text {ann }}=645^{\circ} \mathrm{C}$ and $t_{\text {ann }}=20$ min. The histogram shows three peaks corresponding to NWs seeded from single and agglomerated colloidal NPs as shown by arrows from the SEM micrograph in the inset. (f) Comparison of $\Delta d$ of InAs NWs grown from Au film (stars) and colloids (circles) as a function of $d_{\text {avg. }}$. The squares represent $\Delta d$ obtained from the main Gaussian peak and plotted as a function of $d_{c}$. The shaded region is the relative standard deviation of initial Au colloidal NPs as supplied by the manufacturer. Reprinted with permission from Ref. [92] (Copyright 2015, IOP Publishing). 
These results indicate that colloidal NPs are the catalyst of choice where exact, reproducible NW diameters and very low density are necessary. On the contrary, a reasonable control of NW diameters can also be obtained by dewetting thin film, even though the dispersion grows larger as the diameter increases. The strong advantage of thin film annealing is the wafer-scale uniformity and wafer-to-wafer reproducibility of the NW density and diameters.

\section{Lithography-defined Au disc arrays}

Even though the thermal dewetting method has some advantages in terms of simple preparation, large wafer compatibility etc., the drawback of this technique is the difficulty in obtaining a monodispersed NP ensemble. Another technique widely employed to realize NWs with highly controlled position and dimension is lithography. Lithography is a process of generating (or writing) predesigned patterns on a substrate. A standard lithography procedure includes the following five steps: spin coating, exposure, developing, metal deposition, and lift-off as schematically demonstrated in Fig. 17.

After exposing the resist, the exposed area will be selectively removed by the developer. The temperature of the developer and the developing time are the parameters to be optimized. Slight changes of these two parameters will modify the profile of the resist and significantly change the result of lithography. For example, longer developing times or developing the exposed pattern at higher temperatures will lead to an enlargement of the defined pattern. A simi- lar effect will be obtained by increasing dose during exposure. An optimal develop should lead to open holes with undercut profile in exposed poly(methyl methacrylate) (PMMA), as shown in Fig. 17c. The developed samples are then transferred into a thermal evaporator chamber to perform $\mathrm{Au}$ deposition followed by the lift-off procedure in acetone.

The minimum time to expose a fixed area for a fixed dose is given by the following formula:

$$
D \cdot A=T \cdot I
$$

where $T$ is the time to expose the object (can be divided into exposure time (s)/step size (nm)), $I$ is the beam current, $D$ is the dose and $A$ is the exposed area. In this case, the exposure was always carried out with an acceleration voltage of $20 \mathrm{kV}$ and a probe current of $19 \pm 2 \mathrm{pA}$. Both the exposed area and the dose can be controlled from the ELPHY plus software as a CAD drawing, while the software will calculate the exposure time and feed it to the SEM. SEM images in Fig. 18 show the resulting Au discs generated with electron beam lithography (EBL) by varying doses and defined areas. In the left column the nominal diameter of the $\mathrm{Au}$ discs is $40 \mathrm{~nm}$ in the CAD drawing, but the dose employed during the lithography process increases from the top pattern to the bottom one. By increasing the dose, the resulting Au disc diameters increase from $\sim 37 \mathrm{~nm}$ (Fig. 18a) to $\sim 62 \mathrm{~nm}$ (Fig. 18c). In the right column, the nominal diameter of the Au discs is fixed to $60 \mathrm{~nm}$ in the CAD drawing. Following the same trend, the lithography process

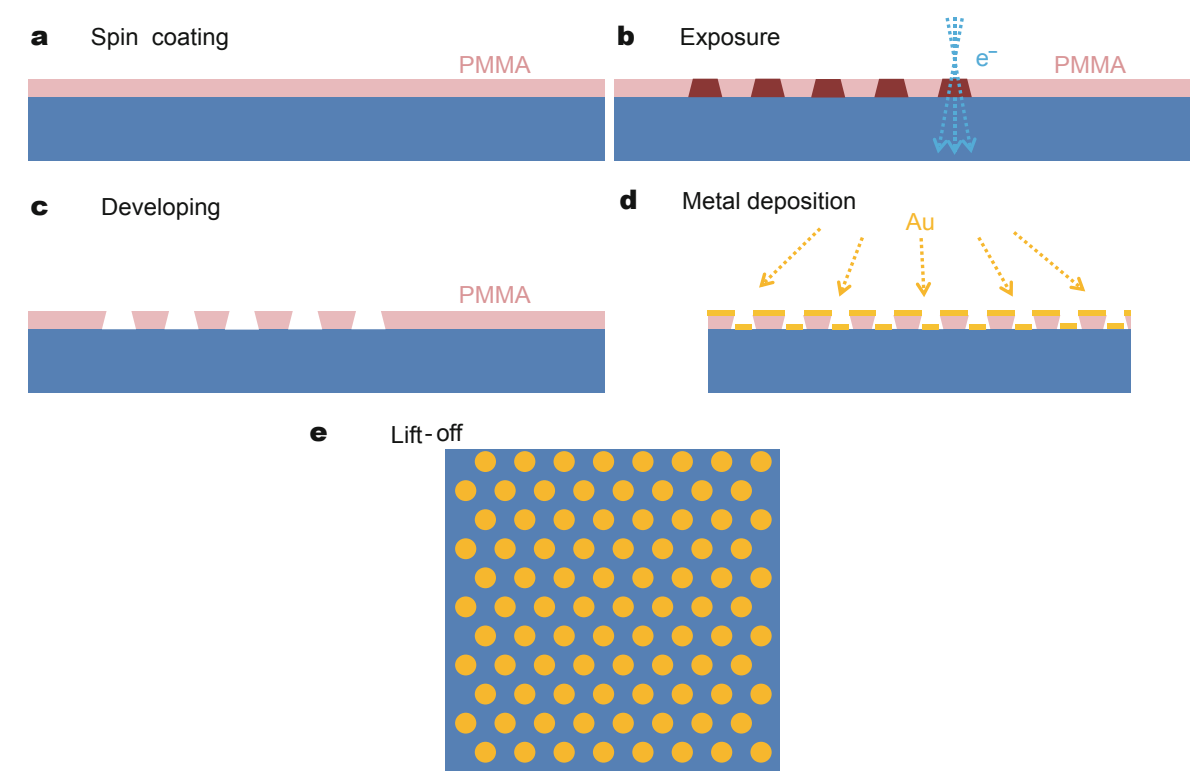

Figure 17 Sketch of the steps employed for the realization of NPs with the EBL procedure. 

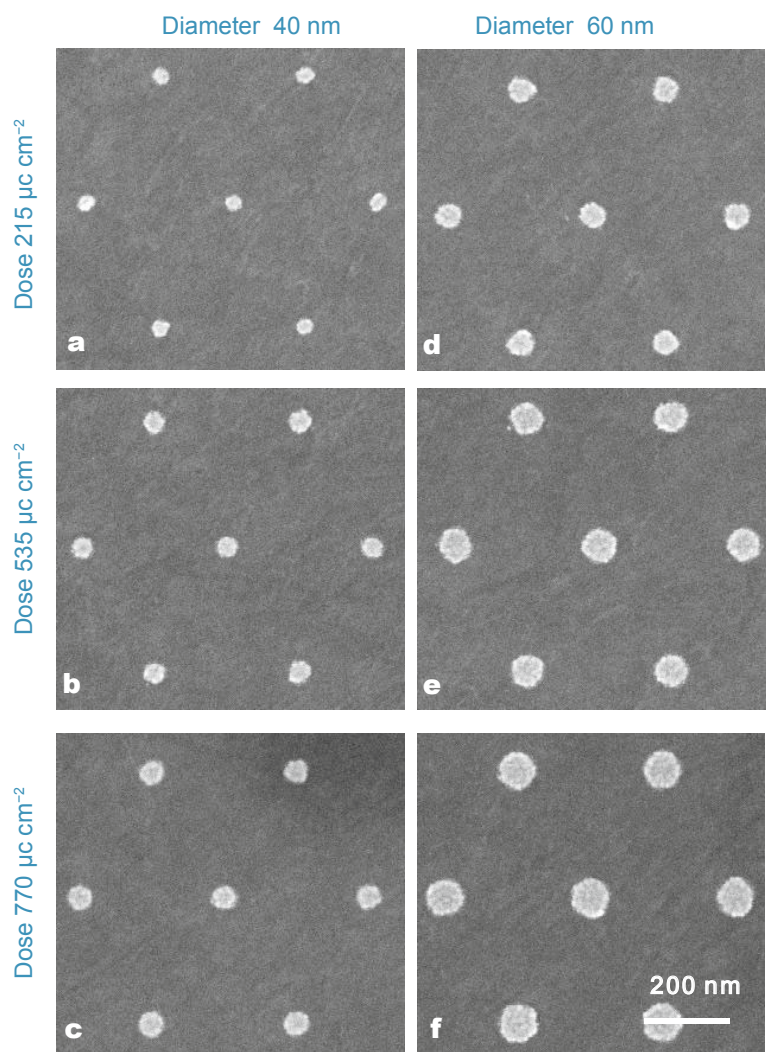

Figure $18 \mathrm{Au}$ discs generated with the EBL process by varying doses and defined areas. The interdistance is fixed to $340 \mathrm{~nm}$. For patterns in $(\mathrm{a}-\mathrm{c})$ the nominal disc diameter is fixed to $40 \mathrm{~nm}$ in the CAD drawing, while for the patterns in $(\mathrm{d}-\mathrm{e})$ the nominal disc diameter is $60 \mathrm{~nm}$.

resulted in Au disc diameters from $\sim 62 \mathrm{~nm}$ to $\sim 87 \mathrm{~nm}$ (Figs $18 \mathrm{~d}-\mathrm{f}$ ) with increasing exposure dose. These results clearly show that the dimension of the Au discs can be controlled by varying either the dose or the defined area. However, higher dose will lead to cross-links inside the PMMA and leave residues on the substrate surface.

The inter-distance between the discs can also substantially change the EBL results. During the exposure, the primary electrons from the SEM are modifying the resist through inelastic scattering with kinetic energy of $E=$ $1 / 2 m_{\mathrm{e}} v^{2}$ and cracking the long carbon chain of the resist into shorter fragments due to $E \gg E_{0}$, where $E_{0}$ is the binding energy of the carbon chains. At the same time, the inelastic collisions of the incident electrons produce a cascade of electrons with kinetic energy greater than $E_{0}$, so called "secondary electrons", which are capable of breaking bonds at distance away from the original collision. The presence of the secondary electrons will spill over into the exposure of the currently written feature, effectively enlarging its image, and reducing its contrast, as shown in Fig. 19a. This
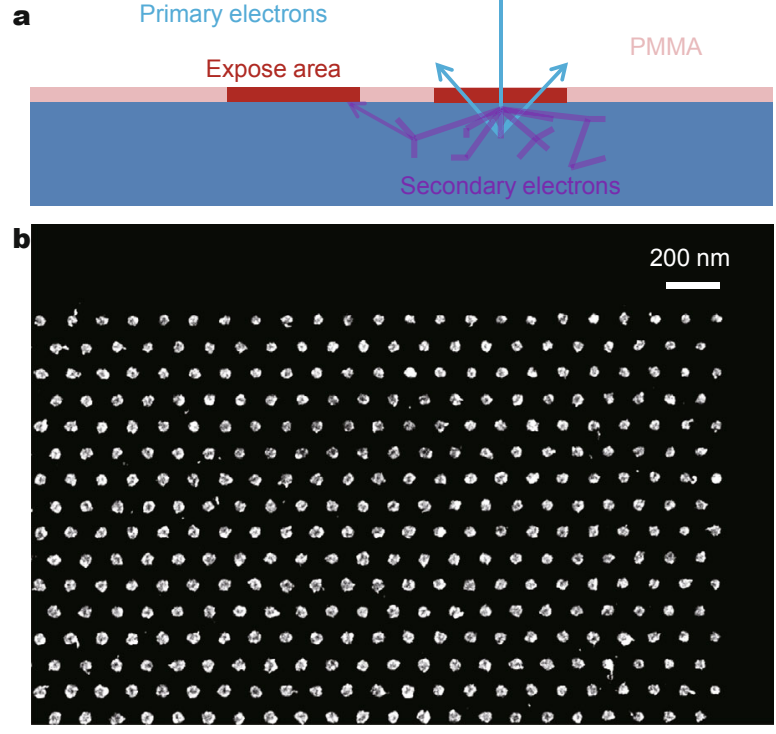

Figure 19 (a) Scheme of proximity effect and (b) SEM image of the boundary of one EBL-defined pattern.

phenomenon, which is usually described as "proximity effect", makes it hard to control the reproducibility of packed features. The corner of an EBL defined hexagonal pattern after lift-off is shown in Fig. 19b. The proximity effect is clear since the Au discs close to the edge of the pattern have smaller diameter than the ones in the center.

\section{Advanced characterization techniques}

In situ and ex situ characterization techniques are also fundamental methods to acquire information on the samples. For instance, advanced electron microscopy can help to visualize both the structure and the morphology of the sample, and diffraction methods allow to reconstruct the crystal structure, etc. These characterization techniques can give insight into the basic physical properties of the material. Additionally, the information is also used as a feedback for the growth procedure, and therefore, will help to optimize the growth parameters. In the following sections several characterization methods will be introduced.

\section{Reflection high-energy electron diffraction (RHEED)}

RHEED is an in situ technique used to characterize crystalline materials. In 2D growth, RHEED systems gather information only from the surface layer of the sample [93]. However, during the growth of NWs, due to the specific growth geometrical configuration, RHEED has been shown to provide valuable information on the NW bulk crystal structure [94]. Thanks to its rapid feedback and the capability of providing the crystal structure information, RHEED is 
a powerful in situ characterization tool employed when the UHV growth systems are employed. A typical RHEED system is normally equipped with remote controls to adjust the beam $x$ - and $y$-position, and beam blanking to avoid the substrate damage induced by the electron beam. The electron gun mounted in the growth chamber can accelerate electrons up to $20 \mathrm{kV}$, and the electrons emitted from the electron gun have kinetic energy $E=1 / 2 m_{\mathrm{e}} v^{2}$. The wavelength of the electrons $\lambda$ is given by $\lambda=h /\left(2 m_{\mathrm{e}} E\right)^{1 / 2}$, where $h$ is Planck's constant. Simple calculation leads to the wavelength of the electron of the order of $10^{-2} \mu \mathrm{m}$, which is two orders of magnitude smaller than the lattice parameter of III-V materials. The electron beam focuses directly on the surface of the sample with a grazing incidence angle less than 5 degrees. In this case, the electron beam travels through the NWs which are grown vertically with respect to the substrate surface. In this geometrical configuration, the main contribution to the diffraction pattern is due to the three-dimensional (3D) volume diffraction inside the NWs. A phosphorus fluorescent screen is mounted on the opposite side of the electron gun, as schematically shown in Fig. 20a. A charge coupled device (CCD) camera is attached to the fluorescent screen and allows to visualize the RHEED pattern (see Fig. 20b) In this way, the diffraction geometry is similar to diffraction obtained by transmission electron microscopy (TEM) but carried out in situ. Combining with diffraction simulation software, it allows to qualitatively analyze the NW crystal structure.

In order to understand the RHEED pattern from the as-grown sample, we have to consider the crystal structure and the growth direction of the NWs. Most of III-V NWs grown by various techniques adopt the hexagonal WZ structure. However, by varying the growth conditions, in terms of III/V flux ratio, growth temperature, catalyst material and diameter, etc., the crystal structure of III-V NWs could be altered to the ZB or to another lower symmetry system. In the following discussion, we are only focusing on the two most common crystal structures found in III-V NW systems: WZ and ZB. In most cases, the free-standing
III-V NWs grow along the [111] orientation of the substrate. Therefore, for the WZ NWs, their [0001] direction is perpendicular to the (111) substrate plane, while for the ZB NWs, their [111] direction is perpendicular to the substrate (111) plane. According to this geometry, we can compare the RHEED patterns from diffraction of WZ along $\{1010\}$ and $\{1120\}$ planes or diffraction from ZB along $\{110\}$ and $\{112\}$ planes.

The typical diffraction patterns acquired from ZB NWs and WZ NWs are shown in Figs 21a, c, e, and g, together with the corresponding calculated patterns (Figs $21 \mathrm{~b}, \mathrm{~d}, \mathrm{f}$, and $\mathrm{h}$ ). It is worth to note that apart from the obviously different appearance of the patterns, the in-plane rotation angle to acquire the patterns is $30^{\circ}$ and equal for both crystal structures. For example, the diffraction patterns of $\mathrm{WZ}$ NWs (Figs 21f and h) will appear as large and small rectangles alternatively by rotating the sample by $30^{\circ}$, while for ZB NWs a rhombus and a rectangular pattern will appear by $30^{\circ}$ rotation [95]. The two obviously different patterns help to identify in situ the crystal structure of the NWs.

Another advantage of the RHEED is that since it is a surface sensitive technique, the application of the RHEED can also offer information of the first stage (nucleation) of the NW growth. For instance, as shown in Figs 22a and b, Tchernycheva et al. [94] observed additional diffraction spots from RHEED after certain thermal annealing process (dewetting). Combining with the ex situ cross-section TEM and selected area electron diffraction (SAED) study (Figs $22 \mathrm{c}$ and $\mathrm{d}$ ), a formation of $\mathrm{Au}_{7} \mathrm{Ga}_{2}$ alloy particles has been confirmed.

\section{High resolution transmission electron microscope (HRTEM)}

Even though SEM images are sufficient to study the morphology of the NWs, they cannot supply any crystal structure information due to the fact that the acceleration voltage is not high enough to make the electrons penetrate the body of the NW. Since the crystal structure influences most physical properties of the material, ex situ TEM investigations are necessary. TEM has a similar electron source as

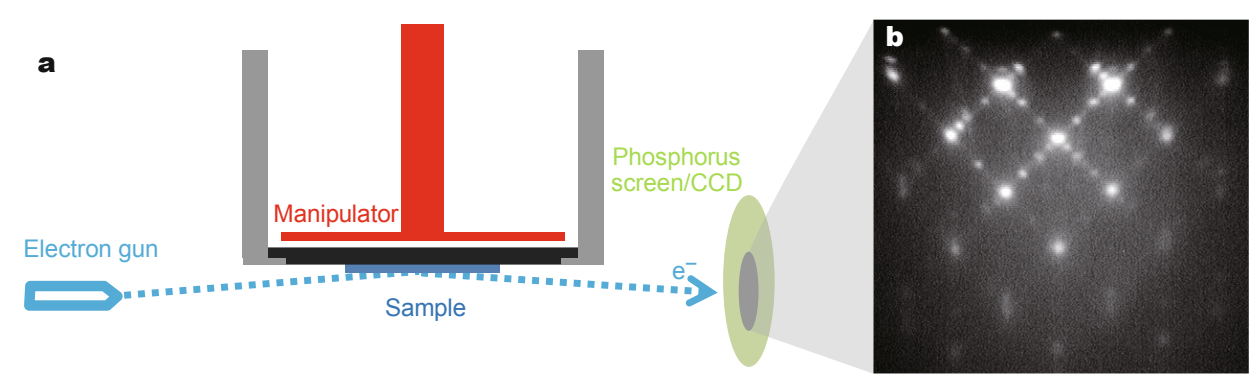

Figure 20 (a) Schematic drawing of RHEED and (b) a typical diffraction pattern of InP NWs grown on InP (100) substrate. 


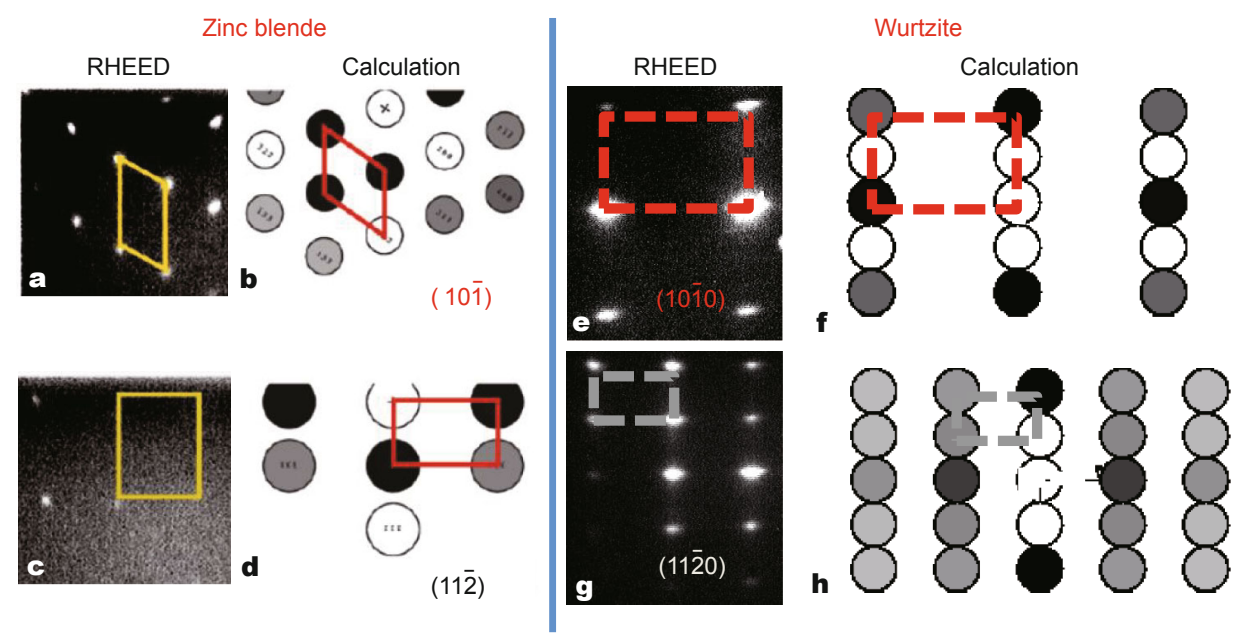

Figure 21 Experimental RHEED and calculated diffraction patterns for both InAs ZB (Reprinted with permission from Ref. [95] (Copyrigh 2010, American Chemical Society)) and WZ structures (Reprinted with permission from Ref. [142] (Copyrigh 2011, American Chemical Society)).
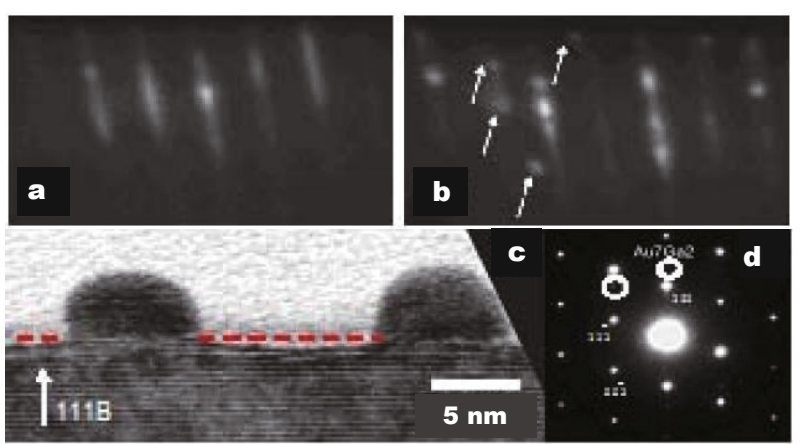

Figure 22 ( $\mathrm{a}$ and b) RHEED patterns of Au deposited GaAs (111) surface after different thermal annealing process. (a) At $500^{\circ} \mathrm{C}$, after annealing; (b) at $300^{\circ} \mathrm{C}$, after cooling. Additional diffraction spots originating from the solid Au-Ga particles are observed. (c and d) Cross-section TEM studies of the alloy catalyst particles after annealing. Reprinted with permission from Ref. [94] (Copyright 2006, IOP Publishing).

SEM but uses a parallel beam to illuminate the specimen, and employs much higher accelerating voltages up to 300 $\mathrm{kV}$. The electrons under such high accelerating voltage have very small wavelength $\lambda$ which is comparable to the atom radius. This allows realizing both diffraction of the lattice and imaging the material with atomic resolution. By employing aberration-correctors, the dimension of the probe can be reduced to about $0.8 \AA$.

HRTEM images are usually used to visualize the inner crystal structure. Combined with the FFT analysis (or SAED), the crystal structure of the NWs can be studied. For example, in Fig. 23 both TEM and HRTEM images of a double InP barrier defined InAs quantum dot embedded in a NW are shown. As shown in Fig. 23a, in the low mag-

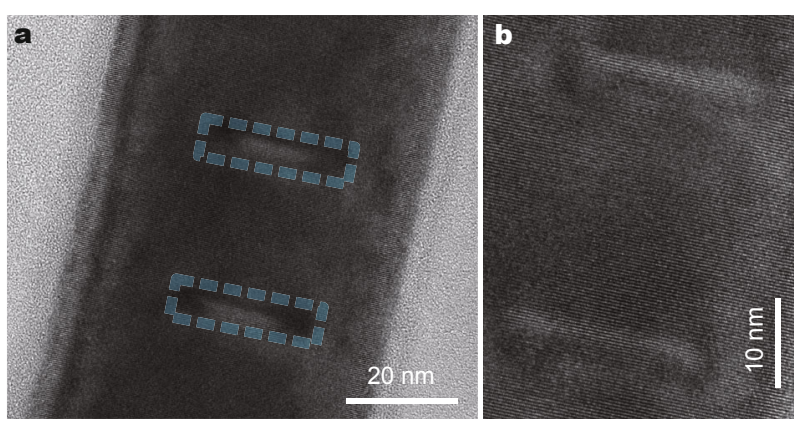

Figure 23 TEM images of an InAs quantum dot embedded in a NW. (a) Low magnification TEM image. (b) High magnification HRTEM image, focusing on the thin InP barriers.

nification TEM mode, the two InP barriers have different contrast with respect to the rest of the NW (InAs). This contrast difference is due to the different lattice constants and the strain induced by the lattice mismatch. In the HRTEM image shown in Fig. 23b, the atoms stacking of these two materials with atomic resolution are clearly visible.

Furthermore, two annual dark field (ADF) and one bright field (BF) STEM detectors are attached to the system and allow performing high resolution HAADF image with resolution of $1.4 \AA$ A. Fig. 24 shows an atomic resolution HAADF image of the tip of a AlAs-GaAs core-shell NW taken from the wurtzite $[2 \overline{1} \overline{1} 0]\}$ zone axis. The dark contrast part is the AlAs core with a diameter of $16 \mathrm{~nm}$, while the bright contrast part in the image corresponds to the 1 $\mathrm{nm}$ thin GaAs shell epitaxially grown on the sidewall of the core. The inset of Fig. 24 is the high magnification image on the sidewall of the core-shell NW. Three MLs of GaAs 


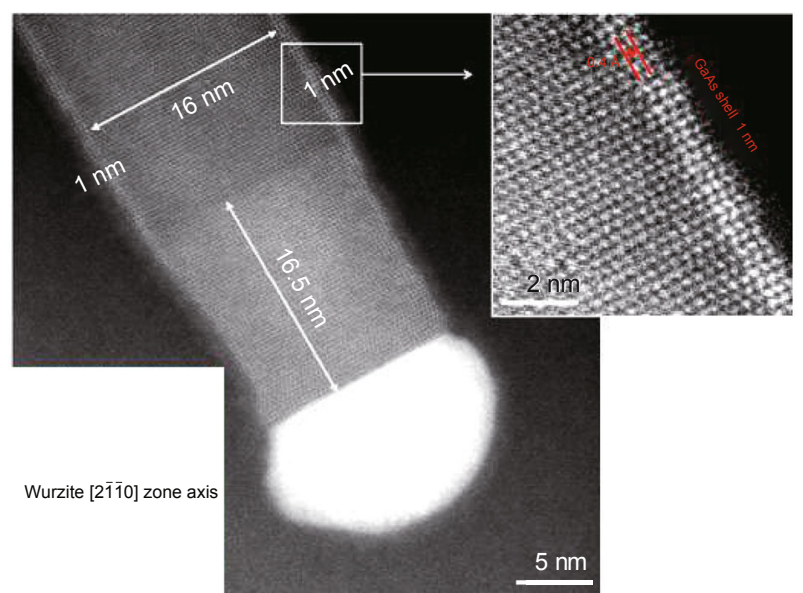

Figure 24 Atomic resolution image of the tip of an AlAs-GaAs core-shell NW with core diameter of $16 \mathrm{~nm}$ and GaAs shell thickness of $1 \mathrm{~nm}$. The zone axis is the wurtzite [2$\overline{1} \overline{1} 0]$.

with plane-to-plane distance of $3.4 \AA$ are distinguishable from the dark AlAs core.

The chemical composition of the NW, interface cleanness, and the chemical composition of the catalyst are important issues. However, the structural analysis mentioned above is not able to perform any chemical characterization of a sample. Therefore, energy dispersive X-ray (EDX) spectroscopy is widely used to determine the chemical composition of NWs, quantitatively [96]. In order to perform an EDX analysis, a converted electron beam is used to induce the X-ray emission from the sample. The X-rays are collected and analyzed by an EDX spectroscopy detector. In Fig. 25a, an STEM image of the tip of an AlAs-GaAs core-shell NW and its EDX analysis are shown. The element map generated by the EDX detector in Fig. 25b shows that there is a pure GaAs segment along the axial growth direction of the core-shell NW, and the catalyst is an alloy of Au-Ga-Al. Furthermore, a sharp interface between the GaAs and AlAs segments is visible from the element map (see Fig. 25b).

\section{Micro-Raman spectroscopy}

Raman spectroscopy is an inelastic light scattering non-destructive technique which allows the access of the phonon modes of materials at the $\Gamma$ point of the first Brillouin zone and in some cases to their dispersion [97-99]. Raman spectroscopy can be experimentally performed at the nanoscale by using a confocal microscope or even a tip enhanced scanning microscope. This allows obtaining a lateral submicron resolution of the properties of a nanostructured material [100] such as a single NW. Therefore, nowadays

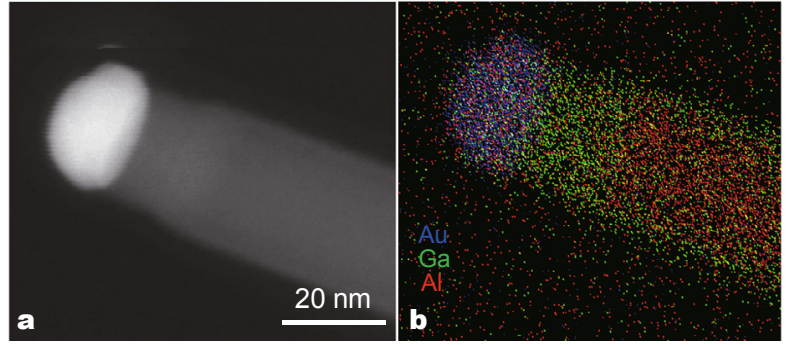

Figure 25 STEM image of the tip of an AlAs-GaAs core-shell heterostructured NW (a), with element map generated by EDX detector (b).

Raman spectroscopy became a versatile and relative standard tool for the characterization of materials giving detailed information on crystal structure, phonon dispersion, electronic states, composition, strain, etc., on thin film and nanostructures [101-109]. In the case of Raman scattering experiments on single NW, a backscattering configuration is preferred. In this configuration, the scattered light is collected along the same direction of the excitation, as shown schematically in Fig. 26 [110].

A cryogenic micro-Raman setup is schematically shown in Fig. 26 to perform Raman experiments on single NW at low temperatures and pressures. The excitation lasers are generated by a HeNe laser for $E_{\text {exc }}=1.959 \mathrm{eV}$, a krypton-ion laser for $E_{\text {exc }}=1.833$ and $1.916 \mathrm{eV}$, and an argon-ion laser for

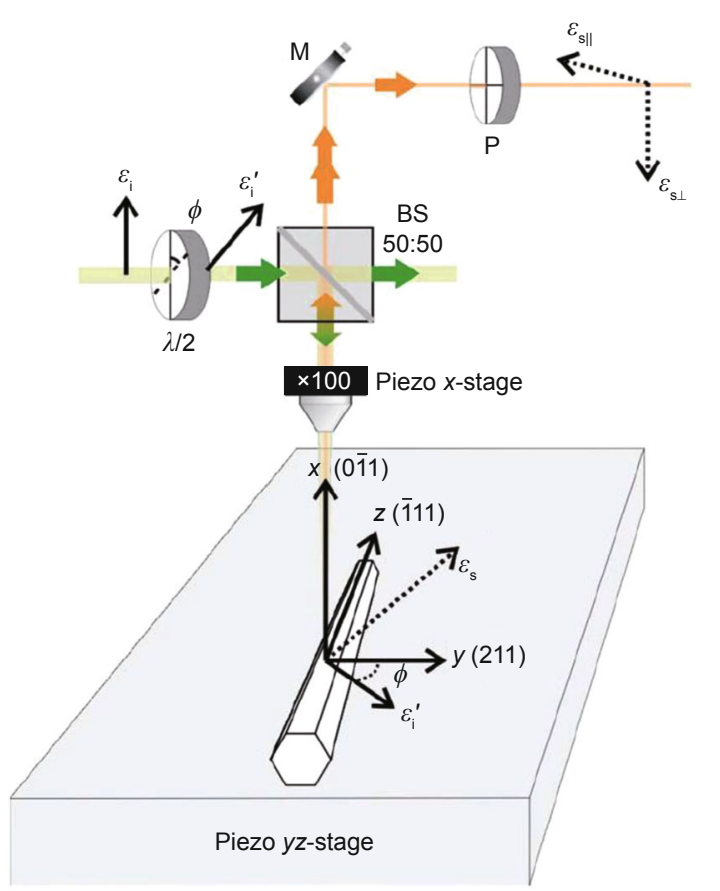

Figure 26 Sketch of the experimental setup and the configuration used for the measurement of single GaAs NW in backscattering geometry. The crystal facets of the NW and the corresponding set of axis used are indicated: $x=[0 \overline{1} 1], y=[211]$, and $z=[\overline{1} 11]$. 
$E_{\text {exc }}=2.410,2.497,2.541$, and $2.708 \mathrm{eV}$. A yellow laser line at $E_{\text {exc }}=2.182 \mathrm{eV}$ is provided by a mixed krypton/argon-Ion laser. All these excitation sources are exclusively gas lasers in order to take advantage of the fine spectral linewidths and are purified by a monochromator and a polarization filter. The sample was positioned on a $X Y$ piezostage inside a Helium-flow-cryostat $\left(10^{-6} \mathrm{mbar}\right.$, at room temperature or in contact with cold $\mathrm{He}$ gas $\sim 10 \mathrm{~K}$ ), which allows the scanning of the surface (and therefore of the NW) with a precision of $10 \mathrm{~nm}$. Polarization dependent measurements are realized as follows. First, the incoming light passes through an $\lambda / 2$ plate so that its polarization $\varepsilon_{\mathrm{i}}$ can be rotated by an angle $\phi$. After passing through a beam splitter (50:50), it is focused on the NW with a $100 \times$ objective ( 0.95 numerical aperture). The polarization of the scattered light $\varepsilon_{\mathrm{s}}$ is analyzed by measuring the intensity of the two components (parallel and perpendicular to the wire). For this reason, a second polarizer is used. The efficiency of the spectrometer depends on the polarization of the incoming light and is higher when it is perpendicular to the slit [110]. In order to avoid artifacts linked to this, a $\lambda / 2$ plate at the entrance of the spectrometer is added in order to flip the polarization of the light into the most efficient direction. Scattered light is collected by an XY Raman Dilor triple spectrometer with a multichannel charge couple device detector in order to achieve a maximum of stray light rejection. The absolute spectral positions of the gratings have been calibrated by $\mathrm{Ne}$ and Xe gas lamps with an absolute error of less than $1 \mathrm{~cm}^{-1}$.

Furthermore, resonance Raman (RR) spectroscopy has been established as a versatile probe for both the structural and electronic properties of semiconductors [111-113]. In particular, RR spectroscopy has been successfully applied to resolve the lattice dynamics and electronic band structures of semiconductor NWs [114-116]. The method relies on the dependence of the Raman cross section on the energy difference between incoming photons impinging on a crystal and absorption centers caused by interband critical points such as the fundamental band gap $E_{0}$. Hence, by examining the excitation dependent Raman cross sections of distinct vibrational modes, one is able to obtain valuable information on the energetic values, dispersions, and symmetries of the bands involved in an optical transition. A particular advantage is the local nature of RR spectroscopy: excited electron-hole pairs involved in the Raman process have not been diffusing towards regions displaying a lower joint density of states prior to their recombination. This is of particular advantage when electronic properties of materials incorporated in nanoscale heterostructures are examined where the fundamental band gap of the mate- rial under investigation is bigger than adjacent layers. In RR spectroscopy measurement, the sensitivity of the $\mathrm{Si}$ charge couple device detector and the three gratings of the triple Raman spectrometer in the setup is a function of $E_{\text {exc }}$ : any optical element will display an excitation dependent transmission and reflection coefficient. By measuring the Raman intensity of a $\mathrm{CaF}_{2}$ single crystal which has been shown to exhibit a constant Raman cross section between 1.8 and $2.8 \mathrm{eV}$ [117], the sensitivity of the setup can be calibrated.

\section{Atom probe tomography}

Due to the complex 3D geometry, large area of interest and low detectability of dopants/impurities, the STEM HAADF or EDS analysis is not a perfect method for these purposes. Recently, atom probe tomography (APT), which is able to offer both $3 \mathrm{D}$ imaging and chemical composition measurements at the atomic scale, are quite often used as a promising tool for this purpose. As a microscope, the APT was invented in 1967 by Müller [118] for the usage in materials science. Similar to the field ion microscopy (FIM) [119], the sample needs to be prepared in the form of a sharp tip. The cooled tip is biased at high direct current (dc) voltage $(5-20 \mathrm{kV})$. The highly small radius of the tip and the high voltage will induce a high electrostatic field (tens $\mathrm{V}$ $\mathrm{nm}^{-1}$ ) at the tip surface, just below the point of atom evaporation. Under laser or high-voltage pulsing, a few atoms are evaporated from the surface by field effect (near 100\% ionization) and then projected onto a position sensitive detector (PSD) with extremely high detection efficiency. The detector measures time of flight mass (TOF) of the ions (i.e., the time between the laser flash and the arrival on the PSD allows to determine the mass-over-charge ratio (m/q)) and the $(X, Y)$ position of the ion impact on the detector simultaneously. The atoms are progressively removed from the tip by repeating the employed laser pause, and a $3 \mathrm{D}$ reconstruction image of the material can be acquired at the atomic scale $[120,121]$.

Fig. 27 shows the detail schematic of a local-electrode atom-probe (LEAP) tomography operated by employing either voltage (I) or laser pulses (II). A microtip specimen array is fabricated on the substrate and applied a positive potential $V_{\mathrm{dc}}$. In order to evaporate atoms as ions, the electric field at one tip specimen has to be increased to a certain value, therefore, the local electrode is pulsed with a negative potential, $V_{\text {pulse }}$. Apart from the application of an electric field, the specimen can also be pulsed by laser pulses to gain a higher repetition rate. The PSD consists of multichannel plates (MCP) in series with a delay-line detector which is used to obtain an FIM image of the atoms 

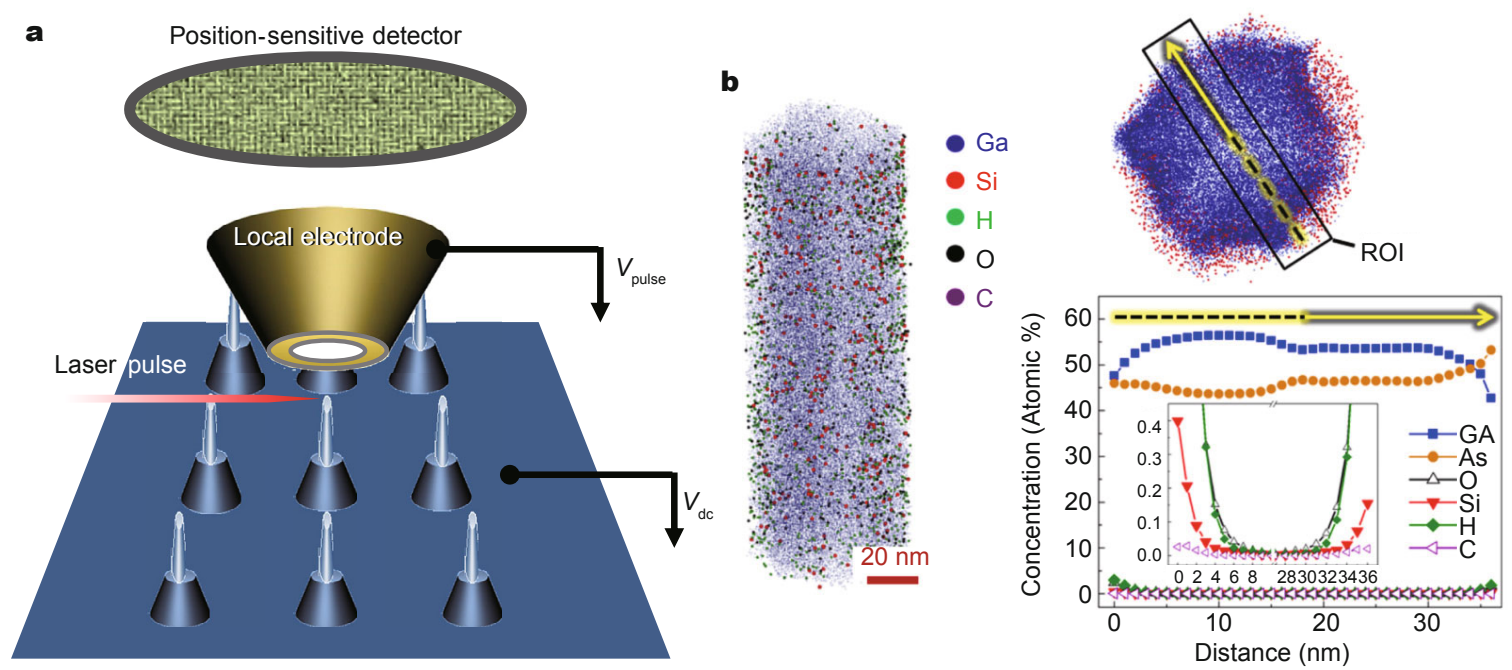

Figure 27 (a) Schematic drawing of a LEAP tomography. (b) Quantitative dopant distribution measured by APT. Reprinted with permission from Ref. [122] (Copyright 2014, Elsevier).

on the surface of a micro tip employing He or Ne as an imaging gas. The distance between the MCP and microtips is variable between 90 and $120 \mathrm{~mm}$ which offers a changeable magnification of an FIM image and/or the TOF distance.

Thanks to the widely application of the dual-beam focused ion beam, position control growth and other sample preparation techniques, the ability to prepare NW-based APT sample has been dramatically improved. Remarkable results of dopants and impurities characterization in single NWs are achieved by APT [122-127] which strongly enriched the research field. As shown by the very recent Ref. [122] (Fig. 27b), quantitative mapping of dopant in the asgrown GaAs NWs can be measured by APT.

\section{SECTION VI: GROWTH OF III-V NANOWIRE HETROSTRUCTURES}

\section{GaAs/AlAs heterostructured NWs}

Conventional lattice matched systems, GaAs/AlAs/AlGaAs as an example, have been demonstrated in either axial [128] or lateral [129-133] heterostructured NWs as it shows in Fig. 28. In particular, the optical quality of GaAs is strongly improved by capping the NWs with a thin AlGaAs shell [128]. For example, a recent report on the optical properties of bare GaAs NWs found that the dc photoresponse of these NWs is dominated by charged surface trap states, the discharge of which may be the source of the slow decaying persistent photocurrent [134]. However, a higher band gap AlGaAs layer can reduce dark current and decrease surface recombination while increasing sensitivity and quantum efficiency. Thin (50-60 nm) AlGaAs capping layers have

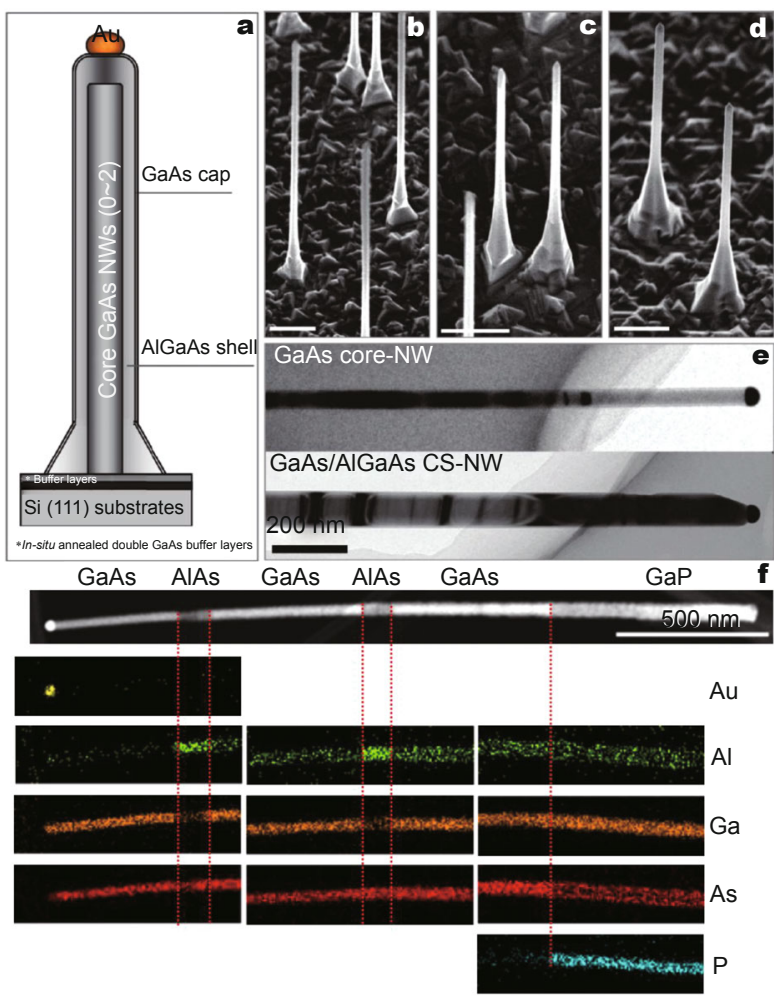

Figure 28 (a) Schematic of GaAs/AlGaAs core-shell NWs with AlGaAs shell grown on $\mathrm{Si}$ (111) substrates coated with GaAs buffer layers; (b-d) SEM images of NWs with different morphology grown under different growth conditions. (e) TEM images of single GaAs core NW (upper image) and GaAs/AlGaAs core-shell NW (bottom image). Reprinted with permission from Ref. [136] (Copyright 2010, IOP Publishing).(f) GaAs/ AlGaAs axial heterostructures grown on GaP stems. Reprinted with permission from Ref. [128] (Copyright 2010, AIP Publishing). 
been shown to be able to improve the time response of photodetector devices to picoseconds [135]. Another remarkable work from Kang et al. [136] demonstrated a control of GaAs/AlGaAs multiple core-shell structure heteroepitaxially grown on Si substrate. As shown in Figs $28 \mathrm{a}-\mathrm{e}$, by a doping GaAs buffer layers and a two temperature growth procedure, they achieved $\mathrm{ZB}$ GaAs/AlAs structure with very high crystal quality. The photoluminescence (PL) measurement of these samples showed an exciton lifetime measured from these NWs are $\sim 600$ ps. Very recently, Persano et al. reported that these core-shell heterostructured NWs have a very good collection efficiency of photogenerated carriers which supports the beneficial role of the shell [137].

On the contrary to GaAs, AlAs is well known as a wide indirect-band gap material. For a long time, the exploitation of AlAs and its alloys in the NW field was mostly limited to their use as shell layer in GaAs NWs [129]. Interestingly, ab initio calculations conducted by De et al. [138] suggested that indirect and large-band gap semiconductors such as AlAs or GaP may display a drastic change of their electronic band structures when transferred from the $\mathrm{cu}$ bic to the hexagonal crystal phase. In the case of AlAs, the authors predict a reduction of the fundamental band transition at the $\Gamma$ point of the first Brillouin zone from approximately 3 to $2 \mathrm{eV}$ due to the introduction of an additional conduction band of $\Gamma_{8}$ symmetry and claim that WZ AlAs exhibits a direct band gap.

Certainly, the implications of such major band structure modifications would be vast in nanotechnology: first, the knowledge on the actual value and the nature of the fundamental band gap in WZ AlAs plays a crucial role for the design of future $\mathrm{GaAs}-\mathrm{Al}_{x} \mathrm{Ga}_{1-x} \mathrm{As}$-AlAs based nanoscaled heterojunctions that are currently investigated for their potential in electronics and photonics $[139,140]$. Second, a direct nature of the fundamental band gap suggests WZ AlAs itself as a novel optically active material in the visible light region. In this regard, special emphasis should be placed on the expected large band gap offset between AlAs in the WZ and $\mathrm{ZB}$ phases that would substantially exceed values reported for other material systems. The assumption of a direct band gap below $2 \mathrm{eV}$ in $\mathrm{WZ}$ AlAs is currently a matter of debate since earlier calculations based on the local-density approximation did not reveal a direct band gap in this energy range [141]. However, little is known on electronic and optical properties of pure AlAs NWs due to its easy oxidization. The first realization of pure AlAs and AlAs-GaAs radial core-shell NWs will be demonstrated by Li et al. In order to prevent the AlAs NWs from fast oxidation, a thin GaAs shell around the AlAs NW has been deposited [142], as shown in Fig. 29. Followed by this remarkable progress, a RR study of single AlAs-GaAs core-shell NW was presented [143], as shown in Fig. 29.

As can be observed in Figs $29 \mathrm{~b}$ and c, a contribution of the $E_{1}$ (TO) mode is present only in the case of an excitation of 1.92 and $1.96 \mathrm{eV}$, while for higher or lower excitation energies, this particular mode is suppressed. This is in agreement with the temperature dependent measurement, which reveals an extinction of the $E_{1}$ (TO) for cryogenic temperatures at $1.96 \mathrm{eV}$. In contrast, the $A_{1}$ (TO) phonon mode displays no particular resonance between 1.84 and $2.5 \mathrm{eV}$ within the experimental accuracy. This can be understood with help of Fig. 29d where the expected electronic band structure at the center of the first Brillouin zone of WZ AlAs is sketched: $\Gamma_{8}$ symmetry is predicted for the lowest conduction band at $1.971 \mathrm{eV}$ with respect to the uppermost $\Gamma_{9}$ valence band [138]. This finding suggests a direct nature of WZ AlAs as the first demonstrate of crystal structure induced indirect-direct band gap transition in III-V semiconductors.

\section{GaP/GaAsP/AlGaP heterostructured NWs}

Apart from GaAs-AlAs system mentioned above, GaP and GaAs are also predicted to have an indirect-direct band gap transition by switch crystal structure from ZB (indirect band gap) to WZ (direct band gap) [138]. In Fig. 30, group of Prof. Bakkers first systematically reported the ability of control as-grown structure of $\mathrm{GaP}$ by varying the growth condition such as growth temperature, V/III ratio [42]. More recently, the same group reported the realization of pure WZ structure GaP NWs and optical measurement of their heterostructures [52]. Due to the fact that the emission wavelength of WZ GaP perfectly fits to the application of green light LED, the GaP-based heterostructrue becomes a hot optoelectronic material.

Similar to InP, the $\mathrm{GaP}$ is often grown with $\mathrm{GaAs}$ as heterostrcutures. For example, GaP/GaAs heterostructures have been used to study the fundamentals of the VLS growth mechanism and distinguish radial from axial growth $[81,144,145]$ as it demonstrated in Fig. 31.

The transition sharpness between the different materials has been investigated as a function of the growth parameters [146]. Borgström et al. [147] demonstrated for the first time that a short segment in a wire can be an optical quantum dot. This was shown by exciton/biexciton emission and antibunching behavior from short GaAs segments in $\mathrm{GaP}$ wires. Following the similar growth route, Zhang et al. reported complex GaP-GaP-GaAs-GaP multiple core-shell structure NWs heteroepitaxially grown on Si substrate, and showed that the GaAs layer is optical activated even though 

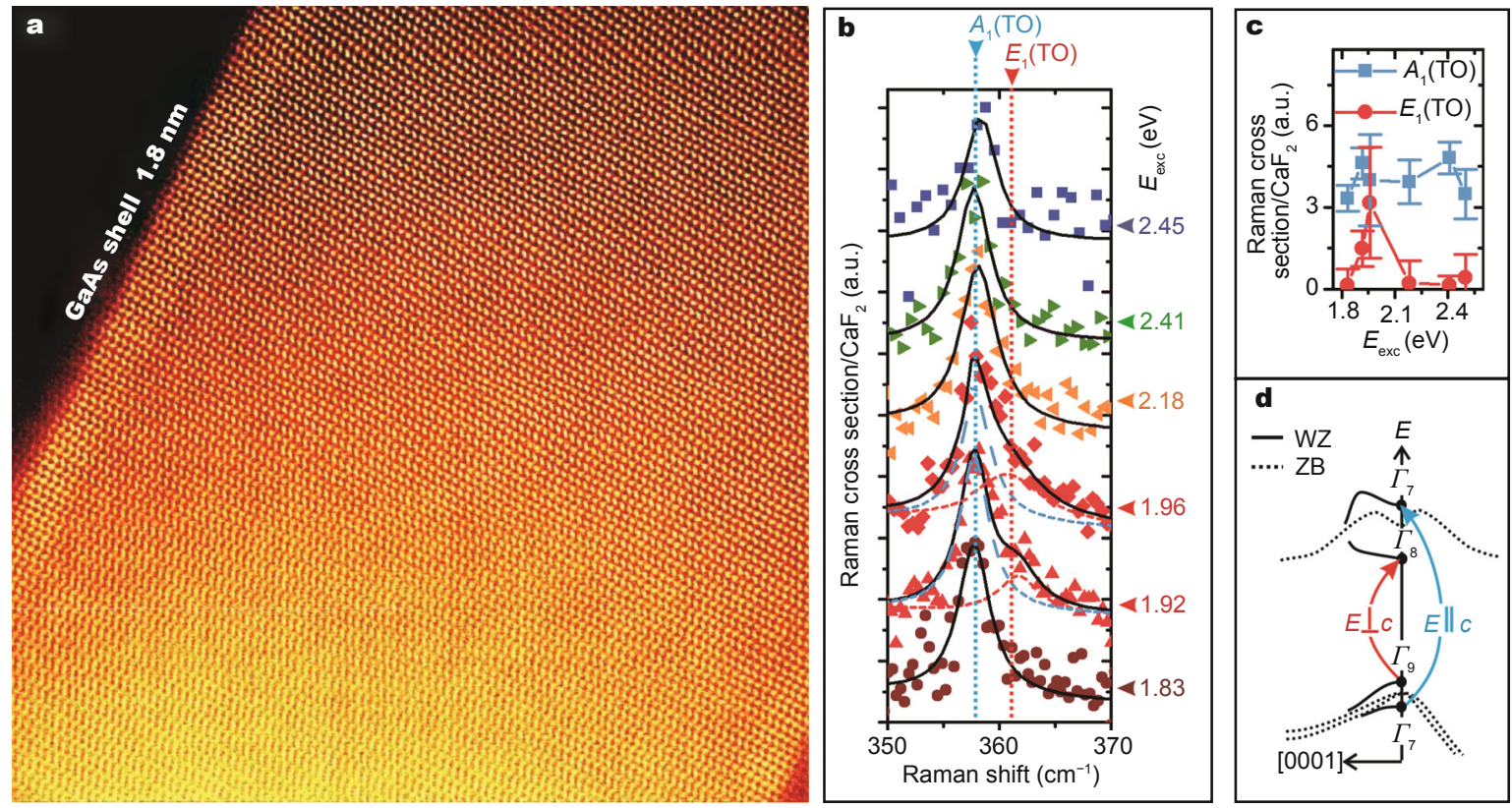

Figure 29 (a) Filtered high resolution HAADF image of the interface between AlAs and GaAs. A $1.8 \mathrm{~nm}$ thin GaAs shell is epitaxially grown on the sidewall of the WZ AlAs NW. (b) Raman spectra collected in the center of an individual AlAs-GaAs core-shell NW. Experimental points and multi-Lorentzian fits are depicted as colored symbols and black solid lines, respectively. Vertical dashed lines are guides to the eye. (c) Raman cross section of the $A_{1}(\mathrm{TO})$ and $E_{1}(\mathrm{TO})$ phonon mode in dependence of the excitation energy. (d) Sketch of the electronic bandstructure in the proximity of the $\Gamma$ point of the first Brillouin zone. Allowed optical transitions in dependence of the polarization of the electric field with respect to the optic axis $c$ are indicated. Reprinted with permission from Ref. [138] (Copyright 2010, American Chemical Society).

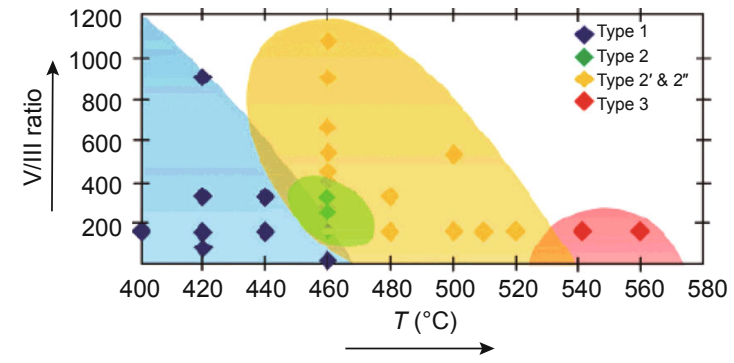

Figure $30 \mathrm{GaP}$ wire morphologies as a function of temperature and V/III precursor flow ratio. Reprinted with permission from Ref. [42] (Copyright 2007, American Chemical Society).

the presence of defects reduced the PL signal significantly [148].

\section{InGaAs based heterostructured NWs}

Thanks to their unique properties, ternary InGaAs NWs are continuously attracting substantial interest in the NW field. Recently, the success of integrating InGaAs and InGaAs-based heterostructures on Si made these materials suitable for many applications such as photovoltaic devices [149], integrated photonics [150-152], tunneling diodes [153], vertical NW field effect transistors [154], and quan- tum information processing [155]. The growth of InGaAs ternary NWs is, however, often not straightforward even though the growth of NWs of this material has been reported to be realized by variety of growth techniques [156]. Growth of InGaAs alloy NWs by conventional VLS method with foreign metal catalyst, i.e., $\mathrm{Au}$, at temperature of $\sim 450^{\circ} \mathrm{C}$ was early reported by Kim et al. [157]. Due to the significant difference in the diffusion, solubility, and incorporation kinetics for indium and gallium, the morphology of the InGaAs alloy NWs resulted in a strong tapering and large dispersion in the length $[157,158]$. Recently, Guo et al. $[159,160]$ provided a detail study on the composition of Au-assisted VLS grown InGaAs NWs. They observed that because of the fact that indium has a higher affinity for the $\mathrm{Au}$ than $\mathrm{Ga}$, gallium is more preferentially to be transported to the axial growth front. During the elongation stage of the NWs, Ga-rich (In)GaAs core region (via VLS-mode) and an In-rich InGaAs shell (via vapor-solid, VS-mode) will be formed simultaneously [161]. In order to overcome these difficulties, catalyst-free growth of InGaAs alloy NWs on Si substrate by MOCVD via vapor-solid mechanism is recently proposed [149]. The overall homogeneity of composition is significantly improved, and the NW morphology is governed by the high anisotropies in 

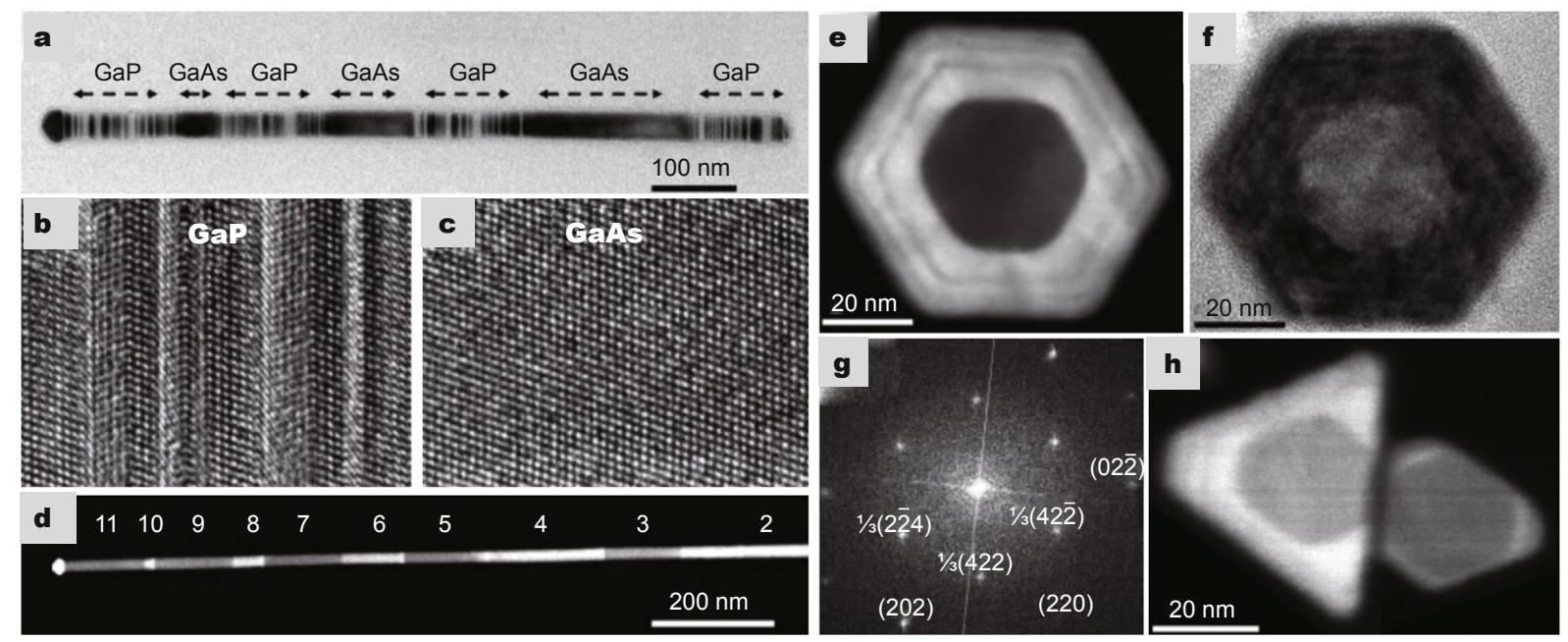

Figure 31 (a) Bright-field TEM image of a heterostructured GaP-GaAs NW. (b, c) HRTEM images of the GaP and GaAs segment. (d) HAADF image of the entire axial heterostructured NWs. (e) Cross section HAADF image of GaP-GaAs core-shell NWs. (f) TEM image of the cross section. (g) Indexed FFT of the (f). (h) HAADF image of two core-shell wires with a triangular shape [81]. Reprinted with permission from Ref. [81] (Copyright 2006, American Chemical Society).

the growth rates of different facets, i.e., higher growth rate along the axial [111] facet than to the $\{110\}$ sidewall facets. The indium composition could be varying from $0.2-1$, and the NW diameter is inversely proportional to the lattice mismatch. Interestingly, the bending of these NWs is often observed, and more detail analysis on these NWs shows a few MLs of compositional fluctuation induced nonuniform strain across the NWs [162]. However, apart from the uniform morphology and composition within single NW measurement, the photoluminescence, and high-resolution XRD spectra always gave a broad linewidth over the whole ensemble NWs. This strongly indicates that there is a large dispersion in NW length, diameter, and composition fluctuations over the as-grown ensemble NWs [149]. Therefore, a catalyst-free, site-selective growth NWs arrays on a substrate with pre-patterned masks, i.e., selective area growth (SAG), became an ideal solution to achieve the high quality InGaAs NWs with uniform composition over the sample $[19,163]$. The template $\mathrm{SiO}_{2}$ masks were defined by either EBL or nanoimprint lithography, and etched by wet etching or sputtering deposition. The growth was carried out at $550^{\circ} \mathrm{C}$ in a solid source MBE chamber, and the relatively higher growth temperature was helping to increase the diffusion and incorporation into the NWs. Morphological compression of SAG growth with conventional catalyst-free growth shows a precise control of NW position and dimension [156], as shown in Fig. 32. High-resolution XRD spectra of InGaAs NWs grown by SAG method showed narrow full-width-at-half-maximum width over wide indium content range [163]. The composition unifor- mity of the SAG grown NWs is also studied by EDS, and STEM carefully [156]. The microstructure of SGA grown InGaAs NWs is studied by HRTEM, selective area diffraction, and atomic resolution HAADF STEM [164] and proved that -similar to other III-As NWs- the WZ phase is the dominated phase and certain density of stacking faults makes the crystal a mixture of WZ and ZB. The length of WZ segment will be reduced by increasing the gallium content and, eventually the crystal will transform into $\mathrm{ZB}$ dominated with gallium up to $36 \%$.

\section{InAs/InP heterostructured NWs}

InAs NW-based heterostructures are mainly fabricated with their phosphides counterparts. Particularly, InP layers embedded in InAs NWs attracted broad interest because the band gap of InP is $\sim 600 \mathrm{meV}$ wider than InAs and therefore, InP can be used as a barrier material to fabricate single electron devices [165-168]. As a matter of fact, InAs NWs were the first of III-V NWs grown by VLS mechanism reported by Yazawa et al. [169] in 1991. By employing the MOCVD method, the NWs were able to grow on $\mathrm{SiO}_{2}$-patterned $\mathrm{GaAs}$ substrates [169] and InAs (111)B substrates with diameter of $20-30 \mathrm{~nm}$ and density of $10^{10}$ $\mathrm{cm}^{-2}$ [3]. However, due to the complexity, the InAs-based heterostructured NWs were realized much later. In 2002, InAs-InP heterostructured NWs were reported grown with UHV CBE system by Bjork et al. $[165,166]$. Trimethylindium (TMIn), tertiarybutylphosphine (TBP) and tertiarybutylarsine (TBA) were used as precursors and the growth were performed at $420^{\circ} \mathrm{C}$. During the growth of the het- 

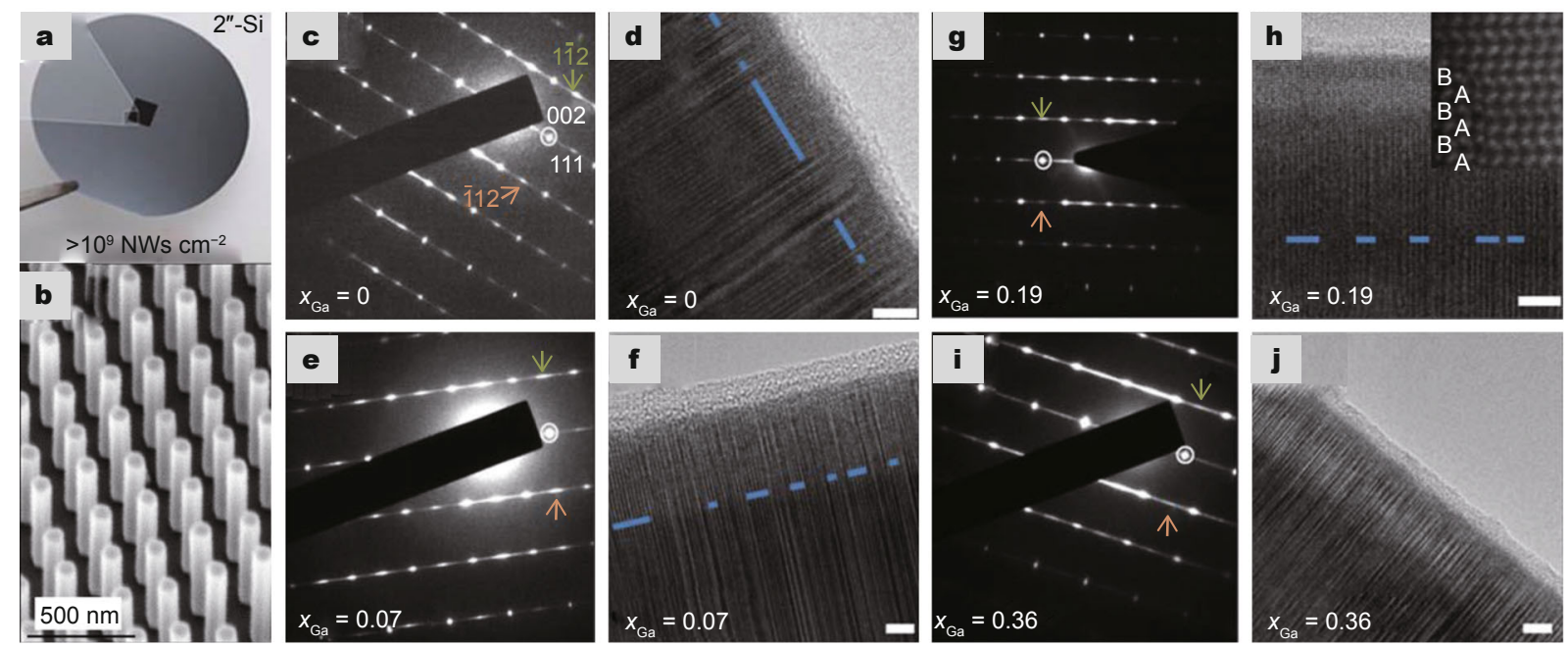

Figure 32 (a and b) Typical optical and SEM image of SAG grown $\mathrm{In}_{1-x} \mathrm{GaxAs}_{x} \mathrm{NWs}$ on nanoimprint-patterned $\mathrm{SiO}_{2} / \mathrm{Si}(111)$ substrates. Reprinted with permission from Ref. [156] (Copyright 2014, Wily-VCH Publisher, Inc.). (c-j) Diffraction patterns and corresponding HRTEM images of SAGIn ${ }_{1-x} G_{x}$ As NWs with different Ga-contents from $x_{\mathrm{Ga}}=0$ (pure InAs) to $x_{\mathrm{Ga}}=0.36$. Reprinted with permission from Ref. [164] (Copyright 2013, American Physical Society).

erostructure, the TMIn source was turned off, the group $\mathrm{V}$ source (TBAs/TBP) turned on after a short pause, and the TMIn turned on again after. HRTEM images show a ML sharpness of the interface attributed to the high vapour pressures of the group $\mathrm{V}$ materials in combination with the low growth rate of $1 \mathrm{ML} \mathrm{s}^{-1}$. Thelander et al. [79] reported the fabrication of resonant tunnel diode (RTD) by using the InP barriers embedded InAs NWs. Via modifying the RTD device dimensions and applying gate electrodes, single NW-based single-electron transistor were also able to be realized $[79,168]$.

As shown in Fig. 33, InAs/InP heterostructured NW is imaged by high resolution HAADF TEM. The image shows two thin InP barriers which have darker contrast and are embedded inside an InAs NW.

However, in a system with large lattice mismatch, e.g., InAs-GaAs core-shell structure NWs, by investigating the interface of the GaAs shell and InAs core with HRTEM, Popovitz-Biro et al. [170] observed a semiperiodic set of edge dislocations emerging from the interface on either side of the core, perpendicular to the growth direction and all along the shell width. Fig. 34a shows clearly three of such dislocations at high magnification, where the emerging point of the dislocations is marked with a white T. Two of the dislocations are further magnified in Fig. 34b. Fig. 34c shows an HRTEM image of a single dislocation which is described schematically in Fig. 34d.

Careful inspection of the HRTEM images reveals that in many dislocations the extra nucleated planes tend to stack in a ZB type stacking. This tendency can be rationalized by the differences between the mismatch values along the [111] axis in ZB (6.6\%) and that of WZ along the corresponding [0001] axis (8\%) [171,172]. Namely, the extra nucleated plane can be more easily accommodated in a ZB type stacking. In fact, the high lattice mismatch-radial $6 \pm 1 \%$ and axial $4 \pm 0.5 \%$ dictates a nonuniform relaxation process and the measured mismatch is found to be different from the nominal mismatch. In order to relax the strain, paired line dislocations and loop edge dislocations within the shell, along the NW, and around it are formed. These defects make the NW partially relaxed [170].

\section{InP/InAsP heterostructured NWs}

InP and InP-based materials have superior gain and low loss properties in the fiber telecommunication band of $\sim 1550 \mathrm{~nm}$ wavelength. Therefore, InP NWs have attracted an increasing amount of attention because of their extensive use in electronics [173], optoelectronics [174,175], optical telecommunications [176-178].

These materials are also good candidates for photovoltaic applications. As opposed to GaAs based material system, InP shows fewer surface states with native oxides. High surface state density can easily deplete carriers in a nanostructure, preventing it from interacting with photons. Therefore, fewer surface states are extremely important to nanostructure devices such as NWs and quantum dots because their surface to volume ratio is large. Both axial $[167,179]$ and core-shell [180] heterostructures have been 


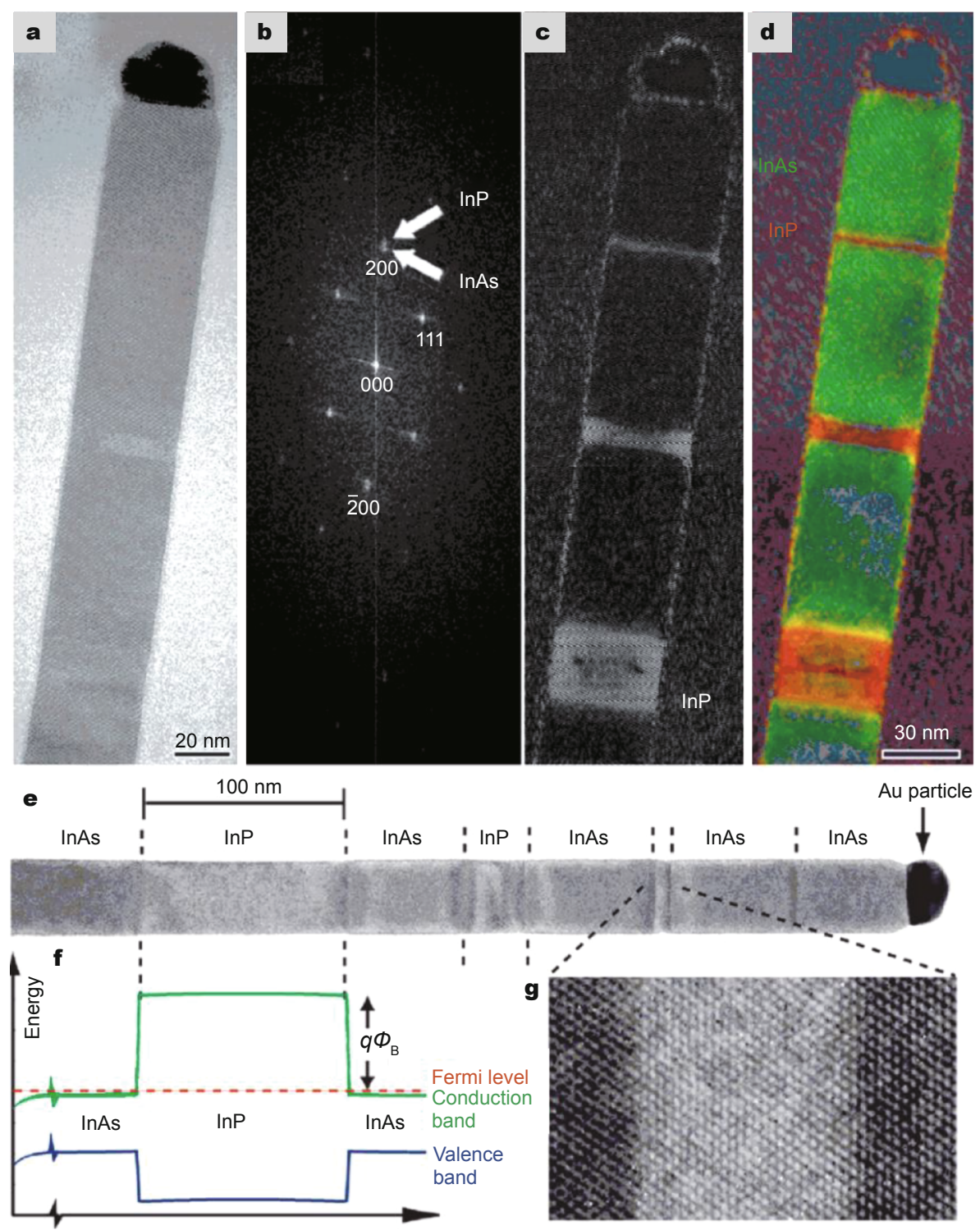

Figure 33 (a) TEM image of a whisker with a diameter of $40 \mathrm{~nm}$. (b) FFT image of (a). (c) Inverse FFT using the information closest to the InP 200 reflection. (d) Colored superimposed image, using an identical mask over the InP and InAs 200 reflection, respectively. (e) TEM image of InP barriers embedded in a InAs NW. (f) Simulated band alignment of the InAs/InP heterostructures, including ideal formation of ohmic contacts to InAs. (g) Atomic resolution HAADF of one InP barrier. Reprinted with permission from Ref. [165] (Copyright 2002, AIP Publishing).

developed for new advanced nanoscale devices. In most of the cases, InP is used as barrier materials with large band gap for the growth of quantum well embedded radial heterostructures. For instance, InP/InAs/InP multiple coreshell NWs are grown by MOVPE on pre-patterend InP substrate (Fig. 35). The essential step to realize the multiple core-shell structure is to switch the growth direction of InP between axial and radial. The phosphorus precursor partial pressure and the growth temperature is demonstrated to be key parameters for this purpose $[28,180,181]$. Very recently, the InP/InAsP/InP radial heterostructures are successfully heteroepitaxially grown on pre-patterned $\mathrm{SiO}_{2}{ }^{-}$ masked Si substrate [182]. Within this study, gas phase of $\mathrm{HCl}$ and $\mathrm{H}_{2} \mathrm{~S}$ is also introduced into the MOVPE reactor as additional parameters to control the radial growth of InP. The room-temperature PL of this radial multiple core-shell structure shows a promising structure for III-V light-emitting device integrated on Si platform. 


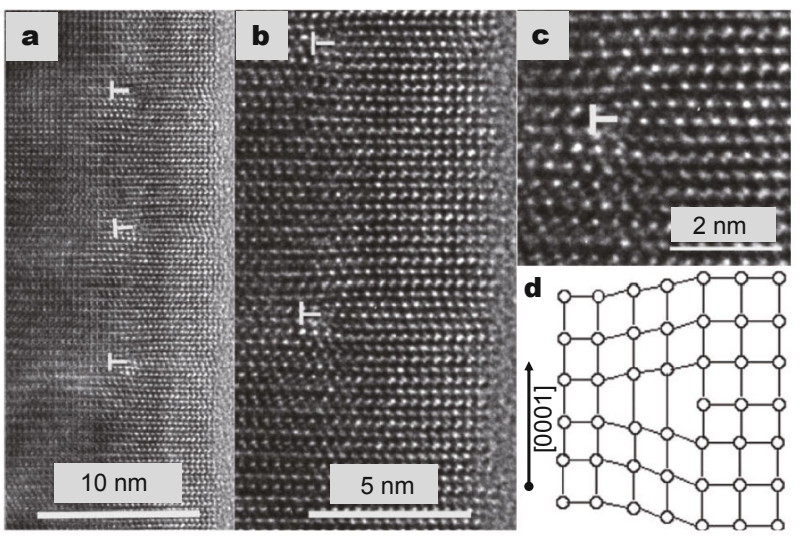

Figure 34 (a) HRTEM image of the core-shell interface region of InAs/ GaAs NW, showing three edge dislocations. (b) Higher magnification of two of the dislocations shown in (a). (c) A single dislocation revealing the extra lattice plane in the shell region (dislocations are marked by " $T$ ") noting that the dislocation's Burgers vector points in the $\langle 0001\rangle$ direction. (d) A schematic drawing of an edge dislocation. Reprinted with permission from Ref. [170] (Copyright 2011, American Chemical Society).

However, most InP-based NWs reported are grown in the direction perpendicular to that of the close-packed planes in the crystal structure, i.e., in the $\langle 111\rangle$ direction for $\mathrm{ZB}$ or the $\langle 0001\rangle$ direction for wurtzite (WZ), in which, the NWs commonly have planar stacking faults (SFs) leading to a faulted crystal or even a mixture of $\mathrm{ZB} / \mathrm{WZ}$ crystal structures [31,183-187]. Although SFs have been found to contribute to many phenomena $[31,187]$, they could significantly affect the electronic $[185,188,189]$ and optical properties [190] of NWs. NW growth in the $\langle 100\rangle$ direction, which has seldom been reported for InP [191], is of particular interest since such wires are inherently free from SFs and represent an ideal ZB single crystal. In 2013, Wang et al. [30] demonstrated that high yield of $\langle 100\rangle$-oriented InP arrays can be achieved simply by changing the filling of $\mathrm{Au}$ droplet before the elongation of NWs. Moreover, by similar strategy, so called "catalyst engineering" the growth direction of the InP NWs can be switched between $\langle 100\rangle$ and $\langle 111\rangle$ direction [38]. The growth direction switching overcomes the defect-limited InP NW-based device fabrication.

\section{InAs/InP/InSb heterostructured NWs}

Another class of materials, antimonide-based compound semiconductors which usually have a narrow band gap, are widely regarded as the first candidate material for the fabrication of third generation infrared photon detectors [192] and integrated circuits with ultra-high speed and ultra-low power consumption $[193,194]$. In contrast to the AlAs-GaAs material system, antimonide semiconductors have large lattice mismatch with other widely employed semiconductors. For instance, InSb, which has the smallest band gap of all binary III-V compound semiconductors, has a lattice mismatch of $19 \%$ with $\mathrm{Si}, 15 \%$ with GaAs, $10 \%$ with InP, and $7 \%$ with InAs. This makes the $2 \mathrm{D}$ growth of this material an extremely difficult task. However, thanks to the easily released strain in NW systems, InAs/InSb axial heterostructured NW has recently been reported $[46,47,195]$. In addition, InSb [196] NWs and InAs [197] NWs have been used to detect Majorana Fermions.

Antimonide compound semiconductor materials, such as InSb, are ideal candidates to fabricate magnetoresistors [198], infrared detectors [192], and high-speed devices $[193,194]$ due to their smallest band gap among all III-V semiconductors, highest bulk electron mobility [199], largest Land'eg-factor [200], and strong spin-orbit interaction. Furthermore, according to the theoretical studies on thermoelectric properties of III-V semiconductors, InSb is also considered to be the best choice for thermoelectric applications due to its small effective mass [201,202].

The fabrication of InSb-based devices has been hindered by its large lattice mismatch with the most widely employed III-V semiconductor substrates. However, the growth of NWs allows accommodating the interfacial strain even for materials with very large mismatch, generally without the formation of any defects. Therefore, the NW structure opens the possibility to realize heterostructured systems by combining InAs and InSb. Several groups have reported the successful growth of InSb $[203,204]$ and InAs/InSb NW heterostructures $[46,47,195]$, enabling the fabrication of InSb and InAs/InSb NW based devices [200,205-208]. Furthermore, Plissard et al. [209] reported a first study on MOVPE growth of InP/InAs/InSb arrays.

If the gas phase species reaching the NW are changed during growth, the thermodynamics of the system changes, and its chemical potential is modified. This may lead to a changing of the contact angle as well as of the dimension of the catalyst particle and eventually changes the dimension of the as-grown NW (as shown in Fig. 4c). InAs/InSb axial heterostructure is an typical example of this effect [46-48]. As illustrated in Fig. 36, the InSb segment of the axial heterostructured NW is larger in diameter than the InAs segment due to the increase of In content in the catalyst particle when the growth is switched from InAs to InSb. Furthermore, this change can also modify the crystal structure of the different segments, as the HRTEM images of the interface of an InAs/InSb heterostructured NW show in Figs 36a and b. Fig. 36a shows contrast due to different lattices which belong to the InAs and InSb segments. In Fig. 36b, a high magnification image is taken at the inter- 

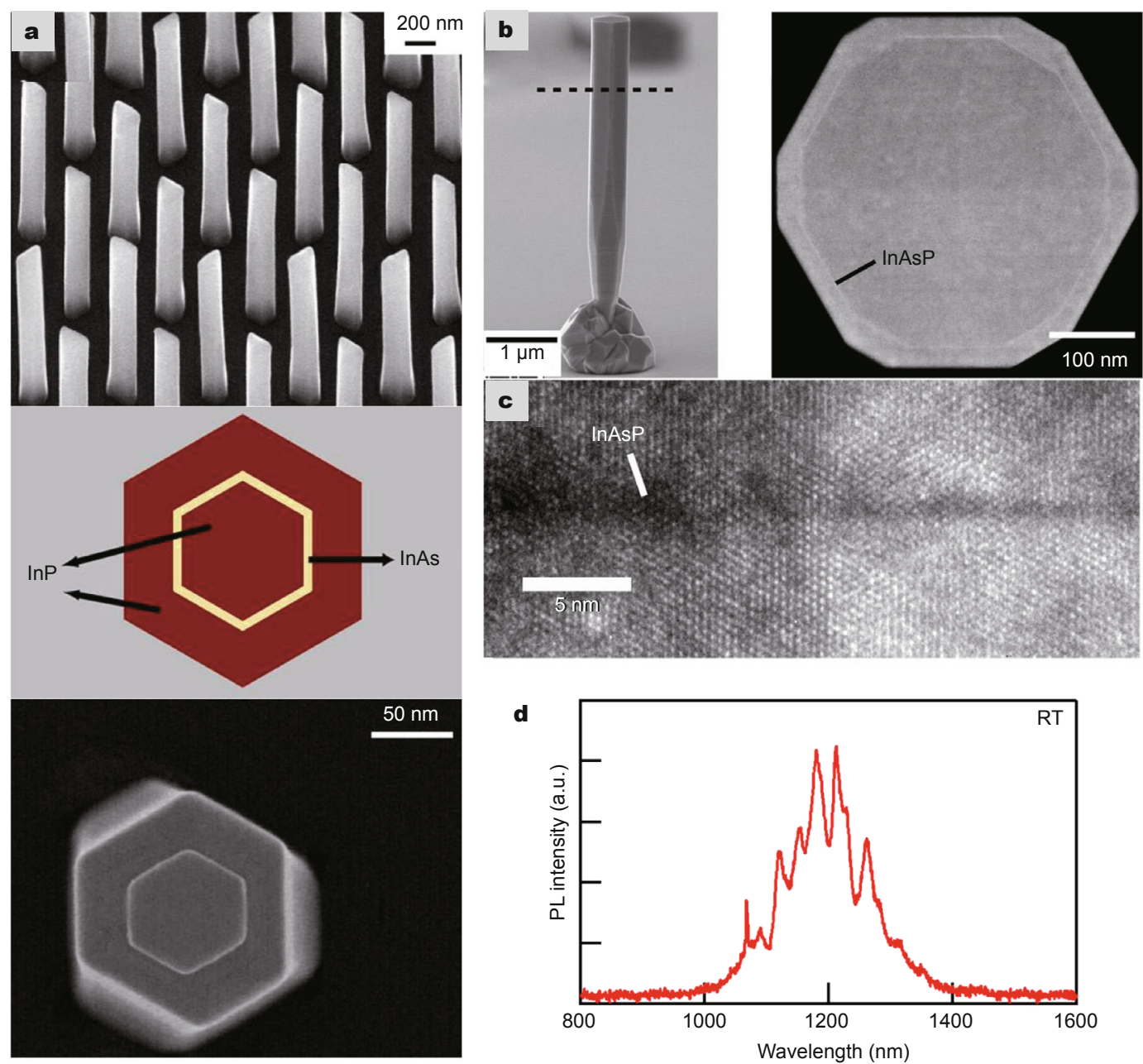

d

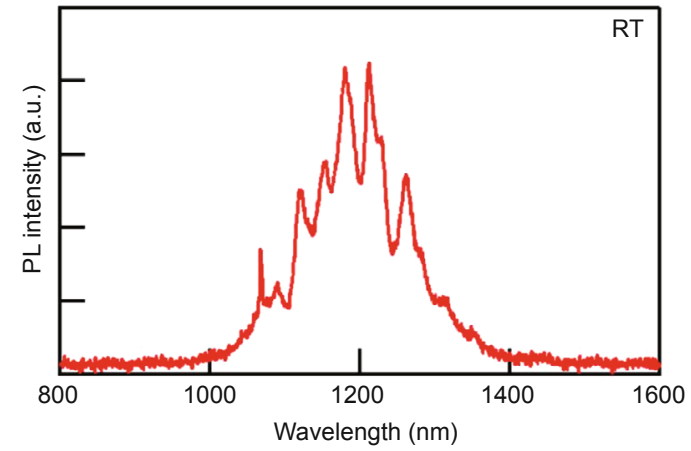

Figure 35 (a) Planary, structure scheme, and cross-sectional SEM of InP/InAs/InP heterostructured NWs grown on InP. Reprinted with permission from Ref. [180] (Copyright 2006, AIP Publishing). (b) Planary and cross-sectional SEM image of InP/InAsP/InP heterostructured NWs grown on Si. (c) HAADF of the InP/InAs/InP interface. (d) Room-temperature PL spectrum of the heterostructue. Reprinted with permission from Ref. [182] (Copyright 2015, AIP Publishing).

face between the WZ InAs and the ZB InSb. An atomically sharp interface is clearly visible. The Fourier analysis shows different periodicity and lattice parameters, see the inset of Fig. 36b. It is worth noting that, a strain induced contrast is visible as well in the TEM image. This strain is introduced by the lattice mismatch between WZ InAs and ZB InSb. However, thanks to the large surface to volume ratio, the strain can be relaxed within a few nanometer region around the interface.

The interfaces between the different material segments of heterostructured NWs and the growth mechanisms are of general research interest [48]. The SEM micrographs of Fig. 36 show two wires viewed from different directions. In Fig. 36c, a NW is viewed along the $\langle 01 \overline{1} 0\rangle$ axis of the InAs stem, and we see three and two facets in the InAs and
InSb segments, respectively. Conversely, in Fig. 36d, a NW is viewed along the $\langle 2 \overline{1} \overline{1} 0\rangle$ axis of the InAs stem, and we see two and three facets in the InAs and InSb segments, respectively. This suggests that the InSb segment has an hexagonal cross section, but is rotated by $30^{\circ}$ around the growth direction, as schematically shown in Fig. 36 . Considering that both an expansion and a rotation of the cross section take place at the initial stage of InSb growth and additional facets, a model is developed by Lugani et al. [48] for the InSb base consisting of low index facets. In this work, they studied the faceting of the InAs/InSb NW in detail by combining SEM and TEM images. As shown in Fig. 36 , the theoretical model agrees well with the experimental observation, and it makes clear understanding of how $\mathrm{ZB}$ InSb segment joints together with WZ InAs stem. 

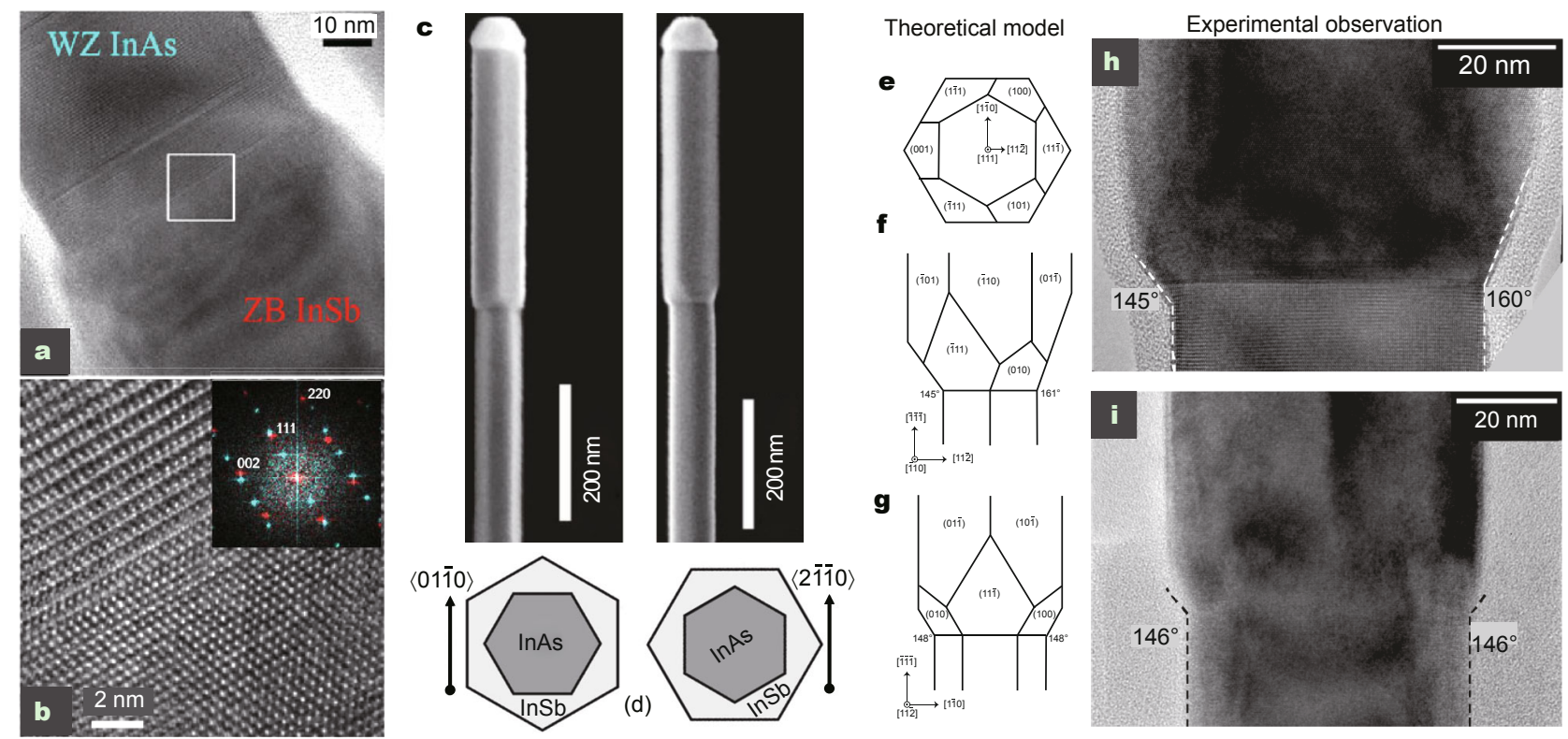

Figure 36 (a) TEM image of the interface of a axial heterostructured InAs/InSb NW. The InAs and InSb segments have different crystal structures which gives different contrast in the high resolution image (b) and different Fourier transform in the inset of (b). (c) SEM image of a NW viewed along the $\langle 0110\rangle$ axis of the InAs stem. (d) SEM image of a NW viewed along the $\langle 2 \overline{1} \overline{1} 0\rangle$ axis of the InAs stem. The bottom panels of (c) and (d) show schematically the cross section of the hexagonal InAs and InSb segments with $30^{\circ}$ rotation around the growth direction. (e-i): Comparison of theoretically predicted model and TEM view of the InAs-InSb interface in an InAs-InSb heterostructured NW. (e-g): top, $\langle 110\rangle$, and $\langle 112\rangle$ views. (h-i): TEM images of the InAsInSb interface of two NWs aligned along (h) $\langle 110\rangle$ and (i) $\langle 112\rangle$ zone axis, respectively. (a and b) Reprinted with permission from Ref. [46] (Copyright 2009, IOP Publishing); (c-i) reprinted with permission from Ref. [48] (Copyright 2010, American Chemical Society).

As demonstrated in Fig. 37, HRTEM micrographs were processed to analyze the strain values and spatial distribution by geometric phase analysis (GPA). Figs 37a and $\mathrm{b}$ report the relative $\varepsilon_{x x}$ and $\varepsilon_{y y}$ strain maps obtained on a typical wire, showing the interface variation of both axial and radial strain. The averaged line profiles of $\varepsilon_{x x}$ and $\varepsilon_{y y}$ across the interface are shown in Figs 37c and d. A step-like change from about 1 (InAs reference) to about 1.063 for $\varepsilon_{x x}$ and 1.07 for $\varepsilon_{y y}$ (InSb segment) is observed, consistent with the $7 \%$ lattice mismatch. The transition is not abrupt but occurs on a scale of 20 to $30 \mathrm{~nm}$, which can be linked to elastic-relaxation effects. These results together with a quantitative analysis of the lattice distances confirm that the InSb segment is nearly relaxed within a few nanometer from the interface which allows us to conclude that an efficient strain relaxation takes place in the system without plastic relaxation thanks to the NW geometry.

The successful growth of InAs/InSb NWs can effectively release the strain and makes the application of InSb become to reality. However, in order to meet different applications, different patterned NWs have to be optimized. For instance, in the application of Majorana fermion detectors, the InSb arrays need to have low density to be able to pick single NW up by nanomanipulator and transferred to the device chip. The work from Plissard et al. [209] on patterned NWs demonstrated in Fig. 38 shows that the growth rate of NWs generally decreases with increasing NW density. Because of the fact that the NWs compete for the incoming material $[17,19,209-211]$, increasing the NW density (i.e., decreasing NW pitch) results in a decrease of available collection area for each NW and thus in the reduction of the NW growth rate. Moreover, the morphology of the InSb is varying by varying V/III ratio from 44 to 166 (Figs $38 \mathrm{a}-\mathrm{d}$ ). It is worth noting that apart from the low growth rate caused by a high NW density, due to the limitation from the GT effect, smaller diameter NWs have even lower growth rate $[54,65,69,212,213]$.

Due to the fact that low dimensional InSb NWs are predicted to have $\mathrm{ZT}>1$ as the reduction of heat transport via phonons due to the diameter of the NWs decrease. In order to achieve $\mathrm{ZT} \sim 3$ at room temperature, it is suggested that InSb NWs of small diameter $(\sim 30 \mathrm{~nm})$ are necessary [201]. Additionally, recent reports on $\mathrm{Si}[214]$ and $\mathrm{Bi}_{1-x} S b_{x}$ [215] NW-based thermoelectric devices have revealed that the high ratio of NW cross-sectional area to device area plays a critical role.

In order to bring InSb NWs into practical thermoelectric applications, it is necessary to achieve small-diameter 
a
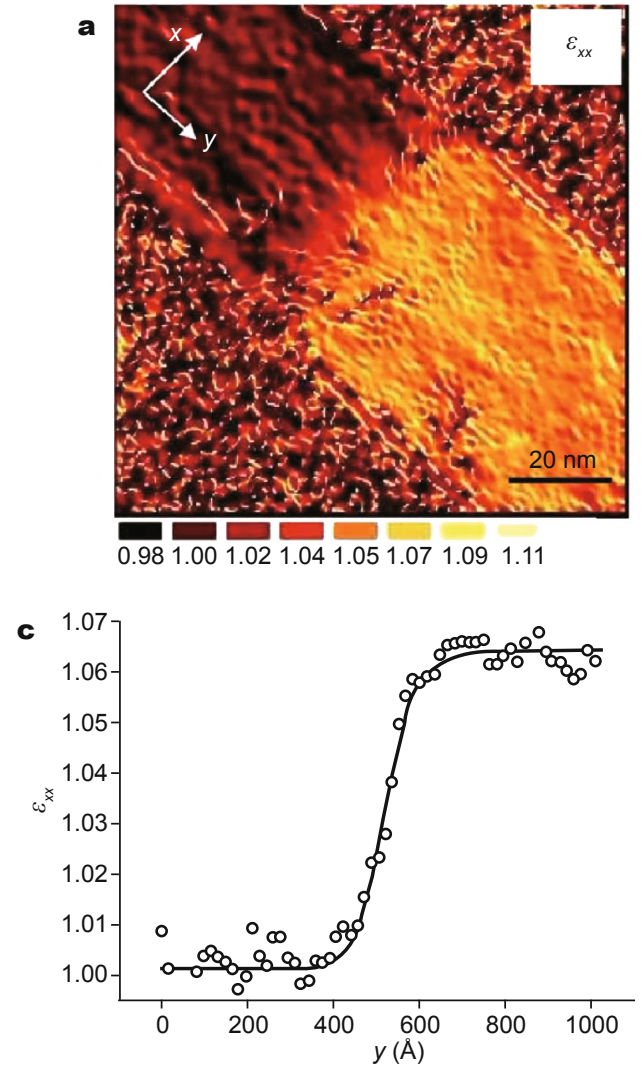

b

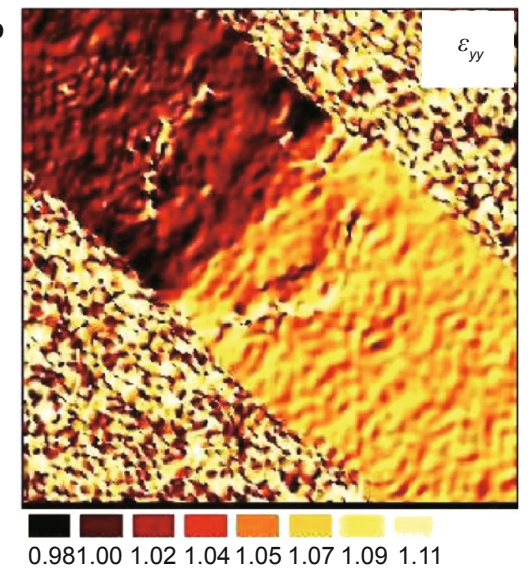

d

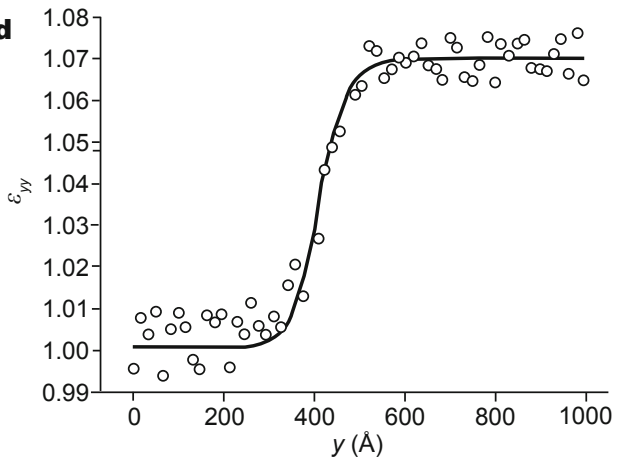

Figure 37 Strain maps (a) and (b) are obtained by geometric phase analysis. The upper (lower) region corresponds to the InAs (InSb) segment. Averaged line profiles of $\varepsilon_{x x}$ (c) and $\varepsilon_{y y}$ (d) across the interface. Reprinted with permission from Ref. [46] (Copyright 2009, IOP Publishing).

NWs with high density. Li et al. [216] shows a contrary behavior of the growth rate by choosing different precursors and under UHV environment. The key strategy is to reduce the adatoms diffusion length $\lambda$ to be much smaller than both the NW length and half of the pitch $a / 2$ [213]. Therefore, the re-evaporated adatom is intercepted and incorporated by a neighboring NW as the scheme shows in Figs $380-$ q. As a result, the growth rate of InSb is proportional to the readsorption probability. In this case, different from the conventional material transport pathway of VLS growth mechanism, the adatom readsorption from the neighboring NWs is the key material transport pathway and this result provides guidelines for growing high density InSb NW arrays with a high growth rate.

Thanks to the precise control of the InP stem growth direction [38], more complex InSb X-, and Y-shape NWs are realized by MOCVD method [217,218]. These structures are fundamental crucial for the incubation of Majorna Fermions device. As it shows in Fig. 39a, the pioneer work was first reported by Plissard et al. [217] by employing a interruption and cool down procedure before the growth of InSb segment. During the cool down procedure, the Au particles would fall down on the sidewall of the stems, and then the substrate was annealed up to the growth temperature to start the elongation of InSb. Therefore, the neighboring NWs have certain possibility to merge and form nanocrosses [217]. In order to increase the yield of InSb nanocross with single crystal junction, the following work from the same group introduced EBL patterned substrates [218] and took advantage of controllable growth direction of InP stems [38].

\section{InAs/InAsSb/InAs double heterostructured NWs}

Among all III-V compound semiconductors, the material having smallest band gap $\left(E_{0}\right)$ occurs in the ternary InAs $\mathrm{Sb}_{1-x}$ alloy systems with values as small as $0.1 \mathrm{eV}$ for $x \sim 0.4$ [219] at room temperature. The values of $E_{0}$ as a function of $x$ for InAs $\mathrm{Sb}_{1-x}$ are plotted in Fig. 40a. The experimental data are obtained from Woolley and Warner [220], Stringfellow and Greene [221] and Dobbelaere et al. [222]. Due to its unique band gap structure and physical properties, In $\mathrm{As}_{x} \mathrm{Sb}_{1-x}$ has become a hot research area. One attractive 


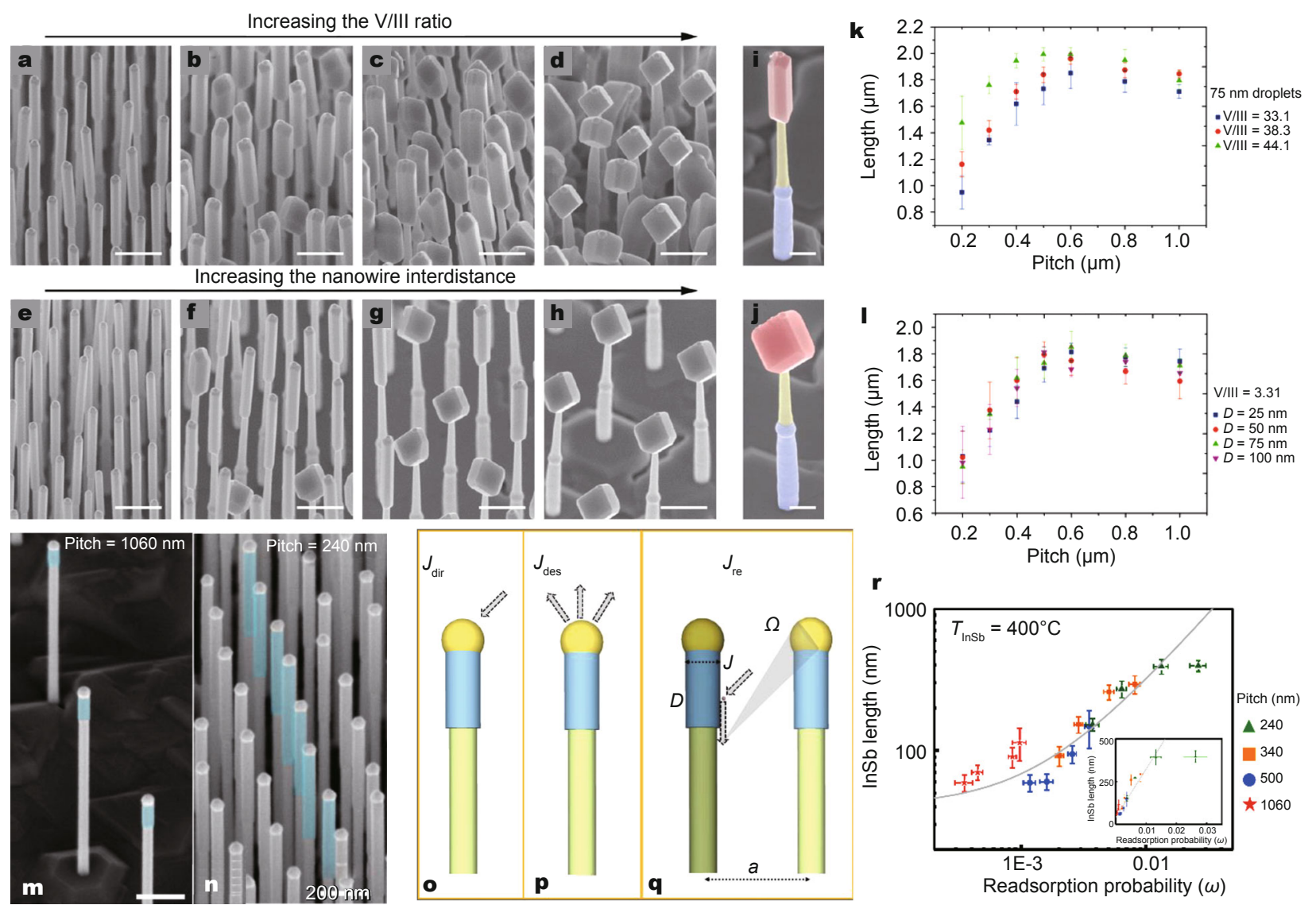

Figure 38 The $30^{\circ}$ tilted SEM images illustrating the evolution of the InSb NW morphology grown from the gold catalyst size of $50 \mathrm{~nm}$. (a-d) NW morphology as a function of the V/III ratio: (a) 44, (b) 66, (c) 88, and (d) 166 for a pitch of $500 \mathrm{~nm}$. Scale bars correspond to $500 \mathrm{~nm}$. (e-h) NW morphology as a function of the pitch: (e) 400, (f) 600 , (g) 800 , and (h) $1200 \mathrm{~nm}$ for a V/III ratio of 44 . Scale bars correspond to $500 \mathrm{~nm}$. (i) NW and (j) Nanocube morphology, colors are indicating different materials. Scale bars correspond to $200 \mathrm{~nm}$. (k) InSb length as a function of the pitch for a droplet size of $75 \mathrm{~nm}$ and different V/III ratios. (l) InSb length as a function of the pitch for a V/III ratio equal to 33.1 and different droplet sizes. (m-n) SEM image for a NW array with pitch of $1060 \mathrm{~nm}$ and $240 \mathrm{~nm}$ reported by Ref. [216]. (o-q) The scheme of re-adsorption assisted growth processes involved in NW growth: (o) direct impingement, (p) evaporation from the droplet, and (q) re-adsorption on the NW sidewalls. (r) Dependence of InSb NW length on readsorption probability $(\omega)$.The gray line is the theoretical fitting. (a-1) Reprinted with permission from Ref. [209] (Copyright 2012, American Chemical Society); $(\mathrm{m}-\mathrm{r})$ reprinted with permission from Ref. [216] (Copyright 2013, American Chemical Society).

application of InAsSb alloys is in the field of environmental gas detection, such as for detecting $\mathrm{CO}_{2}, \mathrm{CO}, \mathrm{CH}_{4}, \mathrm{~N}_{2} \mathrm{O}$, and $\mathrm{O}_{3}$. This field is presently dominated by $\mathrm{HgCdTe}$-based detectors, but the synthesis and the liquid waste of $\mathrm{Hg}$ pose non-trivial health and environmental problems. Another particular advance of this ternary alloy is that $\operatorname{InAs}_{x} \mathrm{Sb}_{1-x}$ has a tunable band gap in the mid-infrared $(2-8 \mu \mathrm{m})$, useful for infrared emission and detection. It is also worth to note that InAs is another outstanding material for device fabrication due to its intrinsic high conductivity and the easiness of fabrication of Ohmic and high transparency contacts [223]. Additionally, InAs has a very interesting broken gap with other antimonide semiconductors, as shown in Fig. 40b. Therefore, for a wide $x$ compositional range, InAs/InAs $\mathrm{Is}_{1-x} \mathrm{Sb}_{x}$ heterostructures have a type II band alignment [224]. The broken gap, along with the absence of strain-related defects typical of NWs, allows an unprecedented flexibility in band engineering [206].

The growth of InAs/InAs $s_{1-x} S b_{x}$ NWs grown by MOVPE over a wide composition range $0.08<\mathrm{x}<0.77$ was demonstrated by Borg et al [225]. They suggested that under the same conditions, due to a decreased effective V/III ratio at the liquid solid interface, the incorporation of $\mathrm{Sb}$ in the NWs could be achieved to a significantly higher level than for planar epitaxy. Latter than the vapor phase epitaxy, InAs/InAs $s_{1-x} \mathrm{Sb}_{x}$ and their double heterostructures were successfully achieved via UHV CBE growth and the $\mathrm{Sb}$ content was increased to $x=0.94$ [226] as shown in Fig. 

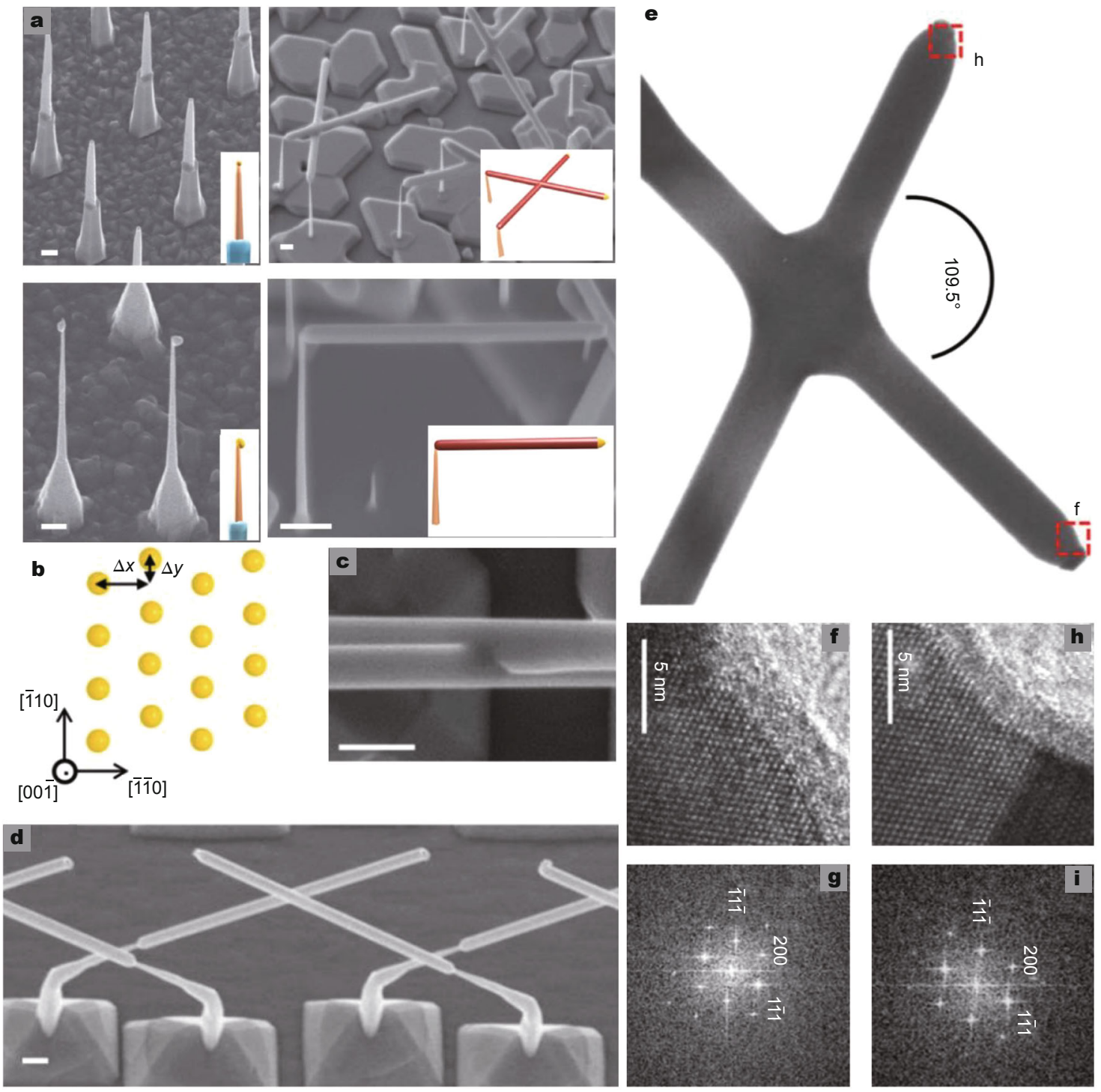

Figure 39 (a) Four-step process for synthesizing InSb NWs crosses, counter clock wise from the left up image Scale bars are $200 \mathrm{~nm}$. Reprinted with permission from Ref. [217] (Copyright 2013, Nature Publishing Group). (b-d) Introducing the misaligned EBL array of gold catalysts which favors crossed NW structures. Scale bars are $200 \mathrm{~nm}$. (e-i) TEM studies of one single-crystalline InSb junction. Reprinted with permission from Ref. [218] (Copyright 2014, Wily-VCH Publishers, Inc.).

41. An ex situ morphological and structural analysis of the InAs/InAs ${ }_{1-x} \mathrm{Sb}_{x} / \mathrm{InAs}$ displays regions of CuPt ordering for low $\mathrm{Sb}$ content (As rich alloy), which was the first time of observation of the CuPt ordering in III-V semiconductor NWs. After proposing the growth mechanism of the lateral growth, the same group reported that in order to suppress the lateral growth of the InAs/InAs ${ }_{1-x} \mathrm{Sb}_{x}$ segments, both growth temperature and InAs stem length have to be optimized [227].

Apart from the Au-assisted growth of InAs/InAs/InAs $s_{1-x}$ $\mathrm{Sb}_{x}$ heterostructures, very recently, InAs, InAs/InAs ${ }_{1-x} \mathrm{Sb}_{x}$, and their homo-/heterostructures heteroepitaxially grown on Si substrate are successfully achieved via self-catalyzed method in UHV MBE chamber [228] and MOVPE reactor [229]. In these cases, In droplets were formed on $\mathrm{Si}$ substrate in order to offer preferable nucleation sites, followed by switching on the As and Sb beam at temperature of $420-470^{\circ} \mathrm{C}$, the NWs would form. The injection of $\mathrm{Sb}$ shows a strongly influence on NW morphology, i.e., axial and lateral growth rate. In terms of crystal phase transition, a detail study from Zhuang et al. [230] shows that from WZ/ZB mixed InAs NWs, addition of Sb led to quasi-pure 

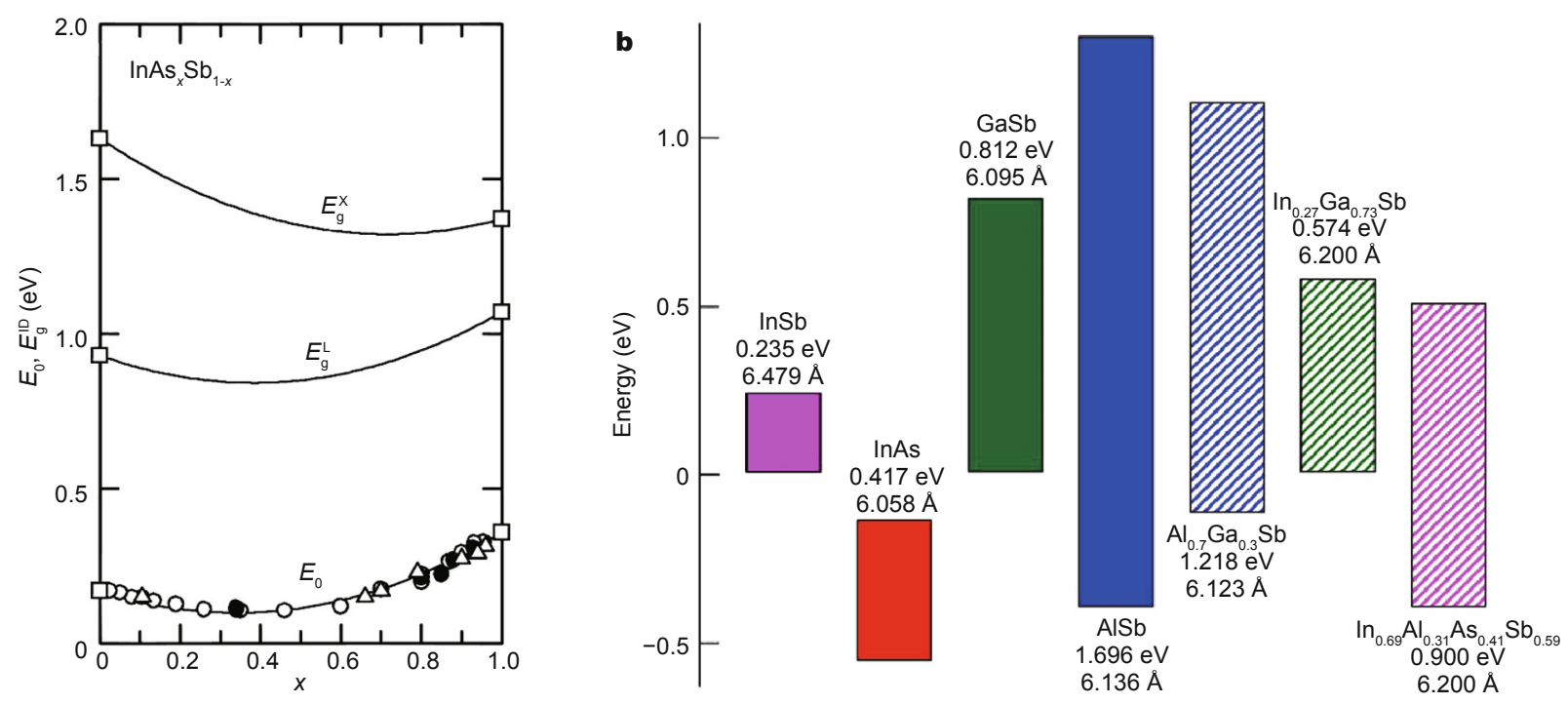

Figure 40 (a) Energies of the lowest direct $E_{0}$ and indirect gaps $E_{\mathrm{g}}^{\mathrm{ID}} v s . x$ for $\operatorname{InAs} \mathrm{s}_{x} \mathrm{Sb}_{1-x}$ at $300 \mathrm{~K}$. The experimental data are taken from Woolley and Warner [220] (o), Stringfellow and Greene [221] $(\Delta)$ and Dobbelaere et al. [222] (•). (b) Band alignments for several binary and ternary alloy semiconductors at 0 K. Reprinted with permission from Ref. [237] (Copyright 2009, John Wily \& Sons).
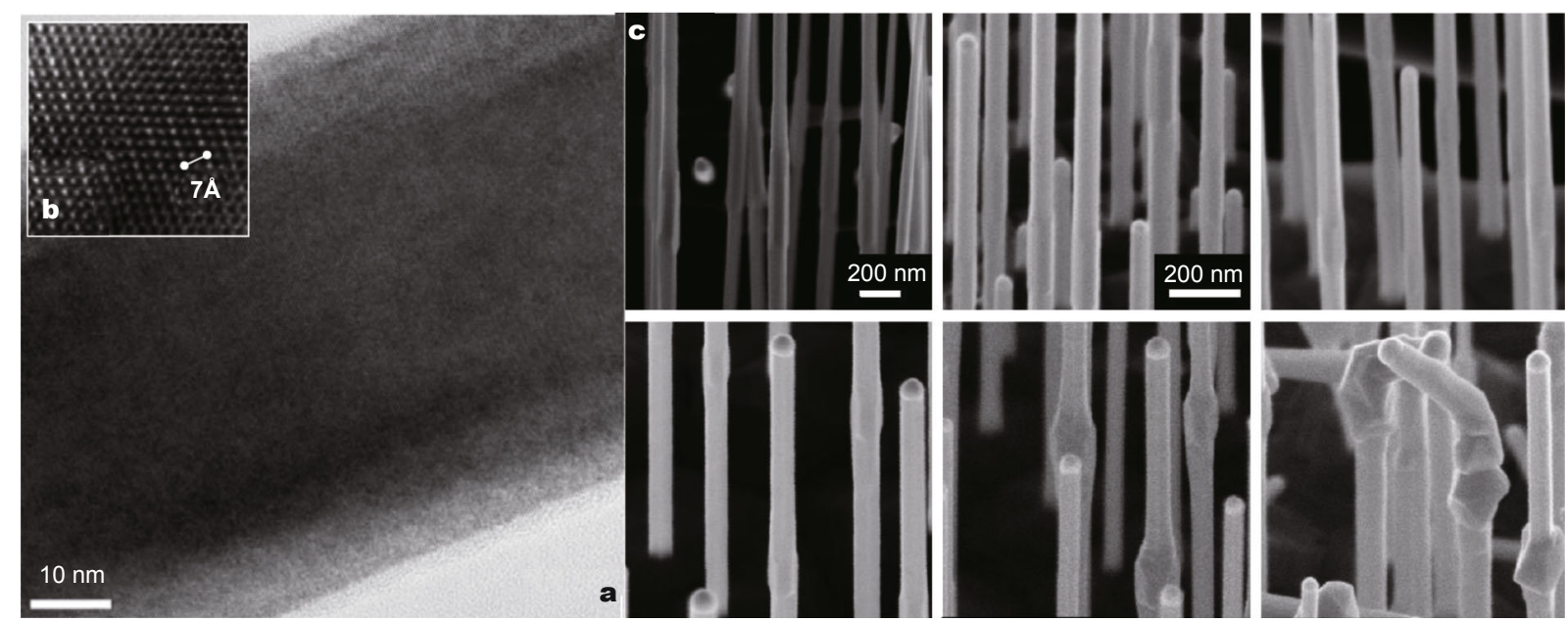

Figure 41 ( $a$ and b) HRTEM image of CuPt ordering in InAsSb NWs. (c) InAs/InAs1- Sb $b_{x}$ double heterostructures grown by CBE. Reprinted with permission from Ref. [226] (Copyright 2012, IOP Publishing).

WZ InAsSb NWs 2-4\%, while further increase of $\mathrm{Sb}$ $(\sim 10 \%)$ resulted in quasi-pure ZB InAsSb NWs.

\section{Branched III-V nanowires}

After development of conventional axial or radial heterostrucures, 3D branched heterostructures become a new attracting structure due to their high surface area and direct transport pathway for charge carriers [219,231]. Additionally, the 3D morphology can effectively improve the light absorption and reduce the reflection and multi-scattering. Therefore, this heterostructures are outstanding candidates for solar cell application. The general idea of the growth of branches is schematically shown in Fig. 42. After the NW stems growth, the fresh catalyst will be deposited on the sidewalls of the NWs, then transferred back into the growth reactor, and start the NW growth from the fresh catalysts. In this case the previous as-grown NWs (trunks) are acting as substrates for new grown NWs (branches) [232-236].

In 2004, Dick et al. [233] first reported the growth of $\mathrm{GaP} / \mathrm{GaAsP}$ double heterostructured branches on $\mathrm{GaP}$ trunks, as shown in Figs 43a and b. The GaP trunks were grown by MOVPE on GaP (111)B substrates, then the $\mathrm{Au}$ 


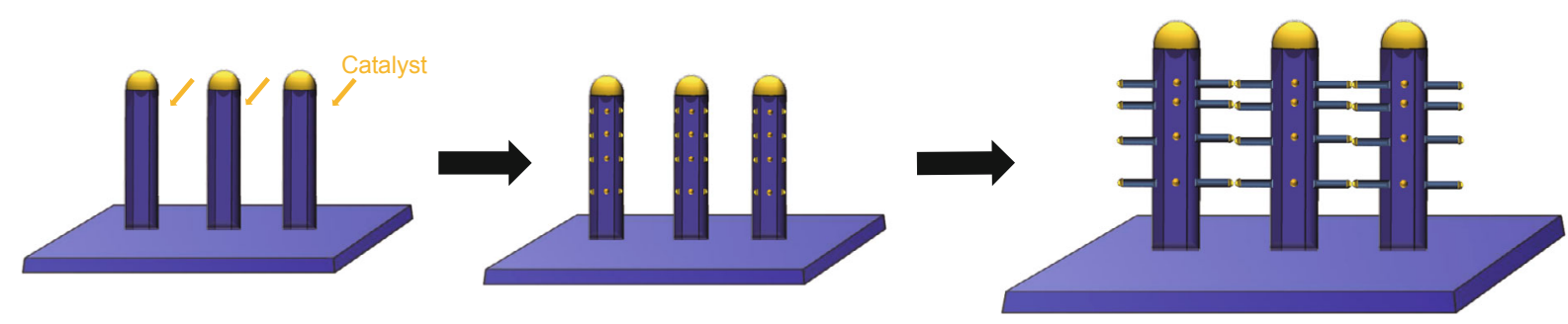

Figure 42 General procedure of branched heterostructures.
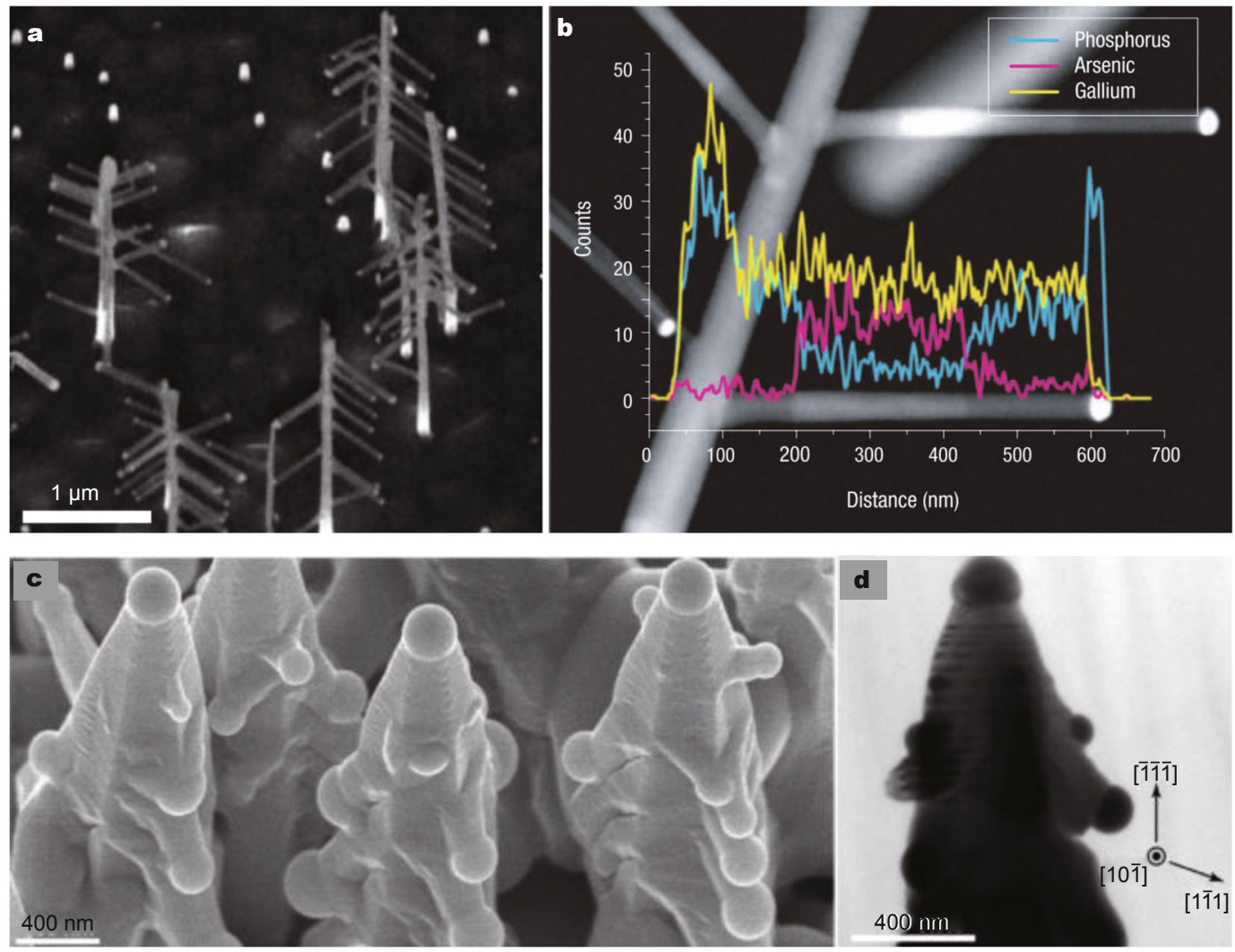

Figure 43 ( $a$ and b) SEM and HAADF (EDS) images of GaP/GaAsP branches grown on GaP trunks. Reprinted with permission from Ref. [233] (Copyright 2004, Nature Publishing Group). (c-d) SEM and TEM image of GaSb nanotrees. Reprinted with permission from Ref. [235] (Copyright 2012, American Chemical Society).

particles were aerosol deposited via electrostatic precipitation along the sidewalls of the trunks. The branced GaP/ GaAsP heterostructures were then grown downwards in the other $\langle 111\rangle \mathrm{B}$ orientation, as shown in Fig. 43b. The number of branches can be controlled by depositing certain number of particles per $\mu \mathrm{m}^{2}$, and the diameter of the branches are determined by the dimension of seed particles. They also showed that the average branch length decreases with increasing branches density but branches length is independent of the position on the trunk. This suggests that during the growth of branches, the surface diffusion of materials over the sidewall of the trunks is not limiting the growth. Interestingly, in the growth of $\mathrm{GaSb}$ trees reported by Yan et al. [235], no additional deposition of seed particles is necessary. The Ga atoms were desorbing from substrate and formed droplets on the sidewall of 1D GaSb NWs with time evolution. Then the GaSb branches grew via self-catalysted VLS mechanism [235]. Similar as the $\mathrm{GaP} / \mathrm{GaAsP}$ branches, the GaSb branches adopt the incline $\langle 111\rangle$ as growth direction, as shown in Fig. 43d. The reflectance measurement of GaSb nanotrees covering substrate shows that the effective absorption of the GaSb 
nanotrees is close to $100 \%$.

\section{SECTION VII: SUMMARY AND OUTLOOK}

Within this review, we first reviewed the conventional VLS growth mechanism and the most widely used growth techniques. Then, we highlighted III-V heterostructures classified by material combinations. Within each materials category, we reviewed their applications and the possible heterostructures achieved by different growth strategies. Several representative experimental results are highlighted within different material systems in order to give an insight view of difficulties, limitations, and the overcoming solutions. From the growth point of view, it is interesting to note that after several years of development from different research groups, more effort recently are put into the realization of complex heterostructures, for instance, crystal phase modifications, multiple core-shell radial heterostructures, NW networks, reversible axial heterostructures, branched heterostructures, etc. Based on the experimental observation, the growth model aiming at understanding of axial and radial growth mechanism and crystal phase controlling is developed both phenomenologically and theoretically. Vice versa, the optimization of overall parameters during the growth of heterostructured NWs are taking into consideration and suggestions from the growth models proposed from theoretical perspective.

For the future development of III-V heterostructures, we foresee several promising fields: (i) self-catalyzed growth and the integration of III-V heterostructured NWs with Si technology. Thanks to the 1D morphology of NWs, the lattice mismatch between III-V and group IV materials (Ge, Si, and C) can be easily overcome. Additionally, the Au-free self-catalyzed growth becomes more friendly to group-IV technology and the lithography processing of III-V and group IV materials is quite similar to each other. The integration of both material systems will make it more promising to fabricate device which has outstanding optical properties form III-V materials and electric properties from group IV materials. (ii) Better control of crystal phase, interface properties including defect properties, diffusion intermixing, and lattice mismatch induced strain engineering. These facts will modify the physical and chemical properties, such as band gap, electric/heat conductivity of conventional III-V materials and open new application fields for heterostructures. (iii) More complex heterostructures meeting requirement of the practical applications. For example, in order to fabricate NW-based optoelectronic devices, quantum computing devices, and energy harvesting devices, a combination of multiple junctions embedded NWs with different materials, dopants, and even foreign functional catalyst NPs is necessary.

Received 6 January 2016; accepted 22 January 2016; published online 29 January 2016

1 Semiconductors International Technology Roadmap for Semiconductors. 2005 Ed. Available from: <http://public.itrs.net/>

2 Wagner RS, Ellis WC. Vapor-liquid-solid mechanism of single crystal growth. Appl Phys Lett, 1964, 4: 89-90

3 Yazawa M, Koguchi M, Muto A, et al. Effect of one monolayer of surface gold atoms on the epitaxial growth of InAs nanowhiskers. Appl Phys Lett, 1992, 61: 2051-2053

4 Yazawa M, Koguchi M, Muto A, et al. Semiconductor nanowhiskers. Adv Mater, 1993, 5: 577-580

5 Haraguchi K, Katsuyama T, Hiruma K. Polarization dependence of light emitted from GaAs p-n junctions in quantum wire crystals. J Appl Phys, 1994, 75: 4220-4225

6 Cui Y, Zhong Z, Wang WU, et al. High performance silicon nanowire field effect transistors. Nano Lett, 2003, 3: 149-152

7 Björk MT, Hayden O, Schmid H, et al. Vertical surround-gated silicon nanowire impact ionization field-effect transistors. Appl Phys Lett, 2007, 90: 142110

8 Bryllert T, Wernersson LE, Löwgren T, et al. Vertical wrap-gated nanowire transistors. Nanotechnology, 2006, 17: S227-S230

9 Tanaka T, Tomioka K, Hara S, et al. Vertical surrounding gate transistors using single InAs nanowires grown on Si substrates. Appl Phys Express, 2010, 3: 025003

10 Fuhrer A, Fröberg LE, Pedersen JN, et al. Few electron double quantum dots in InAs/InP nanowire heterostructures. Nano Lett, 2007, 7: 243-246

11 Hiruma K, Yazawa M, Katsuyama T, et al. Growth and optical properties of nanometer-scale GaAs and InAs whiskers. J Appl Phys, 1995, 77: 447-462

12 Qian F, Li Y, Graděcak S, et al. Gallium nitride-based nanowire radial heterostructures for nanophotonics. Nano Lett, 2004, 4: 1975-1979

13 Hua B, Motohisa J, Kobayashi Y, et al. Single GaAs/GaAsP coaxial core-shell nanowire lasers. Nano Lett, 2009, 9: 112-116

14 Vugt LKV, Rühle S, Vanmaekelberg D. Phase-correlated nondirectional laser emission from the end facets of a $\mathrm{ZnO}$ nanowire. Nano Lett, 2006, 6: 2707-2711

15 Vitiello MS, Coquillat D, Viti L, et al. Room-temperature terahertz detectors based on semiconductor nanowire field-effect transistors. Nano Lett, 2012, 12: 96-101

16 Wu ZH, Mei XY, Kim D, et al. Growth of Au-catalyzed ordered GaAs nanowire arrays by molecular-beam epitaxy. Appl Phys Lett, 2002, 81: 5177-5179

17 Jensen LE, Björk MT, Jeppesen S, et al. Role of surface diffusion in chemical beam epitaxy of InAs nanowires. Nano Lett, 2004, 4: 1961-1964

18 Persson AI, Fröberg LE, Samuelson L, et al. The fabrication of dense and uniform InAs nanowire arrays. Nanotechnology, 2009, 20: 225304

19 Hertenberger S, Rudolph D, Bichler M, et al. Growth kinetics in position-controlled and catalyst-free InAs nanowire arrays on $\mathrm{Si}(111)$ grown by selective area molecular beam epitaxy. J Appl Phys, 2010, 108: 114316

20 Plissard S, Larrieu G, Wallart X, et al. High yield of self-catalyzed GaAs nanowire arrays grown on silicon via gallium droplet positioning. Nanotechnology, 2011, 22: 275602

21 Feiner LF. Nanoelectronics: crossing boundaries and borders. Nat Nanotechnol, 2006, 1: 91-92 
22 Li X, Chen Z, Shen N, et al. Vertically stacked and independently controlled twin-gate MOSFETs on a single Si nanowire. IEEE Electron Device Lett, 2011, 32: 1492-1494

23 Koswatta S, Koester S, Haensch W. On the possibility of obtaining MOSFET like performance and sub-60- $\mathrm{mV} / \mathrm{dec}$ swing in $1-\mathrm{D}$ broken-gap tunnel transistors. IEEE Trans Electron Dev, 2010, 57: 3222-3230

24 Soshnikov IP, Cirlin GE, Tonkikh AA, et al. Atomic structure of MBE grown GaAs nanowhiskers. Phys Solid State, 2005, 47: 22132218

25 Koguchi M, Kakibayashi H, Yasawa M, et al. Crystal structure change of GaAs and InAs whiskers from zinc blende to wurtzite type. Jpn J Appl Phys, 1992, 31: 2061-2065

26 Persson AI, Larsson MW, Stenstrom S, et al. Solid-phase diffusion mechanism for GaAs nanowire growth. Nat Mater, 2004, 3: 677-681

27 Harmand JC, Péré Laperne GN, Mérat-Combes MN, et al. Analysis of vapor-liquid-solid mechanism in Au-assisted GaAs nanowire growth. Appl Phys Lett, 2005, 87: 203101

28 Mohan P, Motohisa J, Fukui T, et al. Controlled growth of highly uniform, axial/radial direction defined, individually addressable InP nanowire arrays. Nanotechnology, 2005, 16: 2903-2907

29 Wood EL, Sansoz F. Growth and properties of coherent twinning superlattice nanowires. Nanoscale, 2012, 4: 5268-5376

30 Glas F, Harmand JC, Patriarche G. Why does wurtzite form in nanowires of III-V zinc blende semiconductors? Phys Rev Lett, 2007, 99: 146101

31 Algra RE, Verheijen MA, Borgström MT, et al. Twinning superlattices in indium phosphide nanowires. Nature, 2008, 456: 369-372

32 Caroff P, Dick KA, Johansson J, et al. Controlled polytypic and twin-plane superlattices in III-V nanowires. Nat Nanotechnol, 2009, 4: 50-55

33 Algra RE, Hocevar M, Verheijen MA, et al. Crystal structure transfer in core/shell nanowires. Nano Lett, 2011, 11: 1690-1694

34 Johansson J, Dick KA, Caroff P, et al. Diameter dependence of the wurtzite-zinc blende transition in InAs nanowires. J Phys Chem C, 2010, 114: 3837-3842

35 Gil E, Dubrovskii G, Andre Y, et al. Record pure zincblende phase in GaAs nanowires down to $5 \mathrm{~nm}$ in radius. Nano Lett, 2014, 14 3938-3944

36 Dick KA, Thelander C, Samuelson L, et al. Crystal phase engineering in single InAs nanowires. Nano Lett, 2010, 10: 3494-3499

37 Lehmann S, Wallentin D, Deppert K, et al. A general approach for sharp crystal phase switching in InAs, GaAs, InP, and GaP nanowires using only group V flow. Nano Lett, 2013, 13: 4099-4105

38 Wang J, Plissard S R, Verheijen M, et al. Reversible switching of InP nanowire growth direction by catalyst engineering. Nano Lett, 2013, 13: 3802-3806

39 Akiyama T, Sano K, Nakamura K, et al. An empirical potential approach to wurtzite-zinc-blende polytypism in group III-V semiconductor nanowires. Jpn J Appl Phys, 2006, 45: L275-L278

40 Pankoke V, Kratzer P, Sakong S. Calculation of the diameter-dependent polytypism in GaAs nanowires from an atomic motif expansion of the formation energy. Phys Rev B, 2011, 84: 075455

41 Braun W, Kaganer VM, Trampert A, et al. Diffusion and incorporation: shape evolution during overgrowth on structured substrates. J Cryst Growth, 2001, 227: 51-55

42 Verheijen MA, Algra RE, Borgström MT, et al. Three-dimensional morphology of GaP-GaAs nanowires revealed by transmission electron microscopy tomography. Nano Lett, 2007, 7: 3051-3055

43 Fortuna SA, Li X. Metal-catalyzed semiconductor nanowires: a review on the control of growth directions. Semicond Sci Technol, 2010, 25: 024005

44 Shi SX, Lu ZY, Zhang Z, et al. Morphology and microstructure of
InAs nanowires on GaAs substrates grown by molecular beam epitaxy. Chin Phys Lett, 2014, 31: 098101

45 Zhang Z, Zheng K, Lu ZY, et al. Catalyst orientation-induced growth of defect-free zinc-blende structured $\langle 00 \overline{1}\rangle$ InAs nanowires. Nano Lett, 2015, 15: 876-882

46 Ercolani D, Rossi F, Li A, et al. InAs/InSb nanowire heterostructures grown by chemical beam epitaxy. Nanotechnology, 2009, 20: 505605

47 Caroff P, Wagner JB, Dick KA, et al. High quality InAs/InSb nanowire heterostructrues grown by metalorganic vapour phase epitaxy. Small, 2008, 7: 878-882

48 Lugani L, Ercolani D, Rossi F, et al. Faceting of InAs-InSb heterostructured nanowires. Cryst Growth Des, 2010, 10: 4038-4042

49 Sun W, Hunag Y, Guo Y, et al. Spontaneous formation of core-shell GaAsP nanowires and their enhanced electrical conductivity. J Mater Chem C, 2015, 3: 1745-1750

50 Oehler F, Gentile P, Baron T, et al. The effects of $\mathrm{HCl}$ on silicon nanowire growth: surface chlorination and existence of a 'diffusion-limited minimum diameter'. Nanotechnology, 2009, 20: 475307

51 Borgström MT, Wallentin J, Trägardh Jo, et al. In situ etching for total control over axial and radial nanowire growth. Nano Res, 2010, 3: 264-270

52 Assali S, Zardo I, Plissard SR, et al. Direct band gap wurtzite gallium phosphide nanowires. Nano Lett, 2013, 13: 1559-1563

53 Berg A, Lehmann S, Vainorius N, et al. Growth and characterization of wurtzite $\mathrm{GaP}$ nanowires with control over axial and radial growth by use of $\mathrm{HCl}$ in-situ etching. J Crystal Growth, 2014, 386: 47-51

54 Mcllroy DN, Alkhateeb A, Zhang D, et al. Nanospring formation-unexpected catalyst mediated growth. J Phys Condens Matter, 2004, 16: R415-R440

55 Wagner RS, Ellis WC. Nucleation of the second phase in the liquid-to-solid transformation of germanium alloys. J Metals, 1963, 15: 76

56 He C, Wang X, Wu Q, et al. Phase-equilibrium-dominated vapor liquid solid growth mechanism. J Am Chem Soc, 2010, 132: 48434847

57 Wacaser BA, Dick KA, Johansson J, et al. Preferential interface nucleation: an expansion of the VLS growth mechanism for nanowires. Adv Mater, 2009, 21: 153-165

58 Foxon CT, Joyce BA. Interaction kinetics of $\mathrm{AS}_{4}$ and $\mathrm{Ga}$ on $\langle 100\rangle$ GaAs surfaces using a modulated molecular beam technique. Surface Sci, 1975, 50: 434-450

59 Yamamoto Y, Stephens KG. A metallurgical investigation of the AuSb and Au-As system. Thin Solid Films, 1979, 60: 123-131

60 Markov IV. Crystal Growth for Beginners. Singapore: World Scientific Publishing Co Pte Ltd, 2003

61 Fröberg LE, Wacaser BA, Wagner JB, et al. Transients in the formation of nanowire heterostructures. Nano Lett, 2008, 8: 3815-3818

62 Johansson J, karlsson CPT, Mårtensson T, et al. Structural properties of $\langle 111\rangle$ B-oriented III-V nanowires. Nat Mater, 2006, 5: 574-580

63 Reif F. Fundamentals of Statistical and Thermal Physics. Long Grove: Waveland Press, 1965

64 Fröberg L. Chemical beam epitaxy of InAs nanowires. Dissertation for Master Degree. Lund: Lund University, 2006

65 Givargizov EI. Fundamental aspects of VLS growth. J Cryst Growth, 1975, 31: 20-30

66 Schubert L, Werner P, Zakharov ND, et al. Probing electrical transport across oxide interfaces by noncontact atomic force microscopy. Appl Phys Lett, 2004, 85: 4968

67 Fröberg LE, Seifert W, Johansson J. Diameter-dependent growth rate of InAs nanowires. Phys Rev B, 2007, 76: 153401

68 Johansson J, Svensson CPT, Martensson T, et al. Mass transport 
model for semiconductor nanowire growth. J Phys Chem B, 2005, 109: 13567-13571

69 Givargizov EI, Chernov AA. Growth-rate of whiskers grown by vapor-liquid-solid mechanism, and role of surface-energy. Kristallografiya, 1973, 18: 147-153

70 Haraguchi K, Hiruma K, Yazawa M, et al. The growth mechanism of nanometer-scale GaAs, InAs, and AlGaAs whiskers. J Electrochem Soc, 2006, 153: C1-C5

71 Dubrovskii VG, Sibirev NV, Harmand JC, et al. Growth kinetics and crystal structure of semiconductor nanowires. Phys Rev B, 2008, 78 : 235301

72 Dubrovskii V, Sibirev N, Cirlin G, et al. Gibbs-Thomson and diffusion induced contributions to the growth rate of $\mathrm{Si}$, InP, and $\mathrm{GaAs}$ nanowires. Phys Rev B, 2009, 79: 205316

73 Cho AY, Arthur JR. Molecular beam epitaxy. Prog Solid State Chem, 1975, 10: 157-191

74 Tsang WT. Chemical beam epitaxy of InP and GaAs. Appl Phys Lett, 1984, 45: 1234-1236

75 Tsang WT. CBE of $\mathrm{Ga}_{0.47} \mathrm{In}_{0.53} \mathrm{As} / \mathrm{InP}$ quantum wells and heterostructure devices. J Cryst Growth, 1987, 81: 261-269

76 Tsang WT. From chemical vapor epitaxy to chemical beam epitaxy. J Cryst Growth, 1989, 95: 121-131

77 Lüth H. Chemical beam epitaxy-a child of surface science. Surface Sci, 1994, 299: 867-877

78 Li J, Wang D, LaPierre RR. Advances in III-V semiconductor nanowires and nanodevices. Sharjah: Bentham Science Publishers, 2011

79 Thelander C, Martensson T, Björk MT, et al. Single-electron transistors in heterostructure nanowires. Appl Phys Lett, 2003, 83: 2052

80 Tchernycheva M, Cirlin GE, Patriarche G, et al. Growth and characterization of InP nanowires with InAsP insertions. Nano Lett, 2007, 7: 1500-1504

81 Verheijen MA, Immink G, Smet T, et al. Growth kinetics of heterostructured GaP-GaAs nanowires. J Am Chem Soc, 2006, 128: 1353-1359

82 Mohseni PK, Maunders C, Botton GA, et al. GaP/GaAsP/GaP core-multishell nanowire heterostructures on (111) silicon. Nanotechnology, 2007, 18: 445304

83 Giermann AL, Thompson CV. Solid-state dewetting for ordered arrays of crystallographically oriented metal particles. Appl Phys Lett, 2005, 86: 121903

84 Kim D, Giermann AL, Thompson CV. Solid-state dewetting of patterned thin films. Appl Phys Lett, 2009, 95: 251903

85 Wang D, Schaaf PJ. Two-dimensional nanoparticle arrays formed by dewetting of thin gold films deposited on pre-patterned substrates. J Mater Sci Mater Electron, 2011, 22: 1067-1070

86 Kan W, Wong H. Fingering instability of a retracting solid film edge. J Appl Phys, 2005, 97: 043515.

87 Danielson DT, Sparacin DK, Michel J, et al. Surface-energy driven dewetting theory of silicon-on-insulator agglomeration. J Appl Phys, 2006, 100: 083507

88 Jiran E, Thompson CV. Capillary instabilities in thin films. J Electron Mater, 1990, 19: 1153-1160

89 Jiran E, Thompson CV. Capillary instabilities in thin, continuous films. Thin Solid Films, 1992, 208: 23-28

90 Wang D, Ji R, Schaaf P. Formation of precise 2D Au particle arrays via thermally induced dewetting on pre-patterned substrates. Beilstein J Nanotechnol, 2011, 2: 318-326

91 Ye J. Solid-State dewetting of continuous and patterned single crystal Ni thin films. PhD Thesis, Massachusets Institute of Technology, 2011

92 Gomes UP, Ercolani D, Zannier V, et al. Controlling the diameter distribution and density of InAs nanowires grown by Au-assisted methods. Semicond Sci Technol, 2015, 30: 115012

93 Braun W. Applied RHEED: Reflection High-Energy Electron Diffraction During Crystal Growth. Berlin: Springer-Verlag, 1999

94 Tchernycheva M, Harmand JC, Patriarche G, et al. Temperature conditions for $\mathrm{GaAs}$ nanowire formation by Au-assisted molecular beam epitaxy. Nanotechnology, 2006, 17: 4025

95 Heun S, Radha B, Ercolani D, et al. Pd-assisted growth of InAs nanowires. Cryst Growth Des, 2010, 10: 4197-4202

96 Goldstein J, Newbury DE, Joy DC, et al. Scanning Electron Microscopy and X-Ray Microanalysis. Berlin: Springer-Verlag, 2003

97 Gõni AR, Pinczuk A, Weiner JS, et al. One-dimensional plasmon dispersion and dispersionless intersubband excitations in GaAs quantum wires. Phys Rev Lett, 1991, 67: 3298

98 Zunke M, Schorer R, Abstreiter G, et al. Angular-dispersion of confined optical phonons in GaAs/AlAs superlattices studied by micro-Raman spectroscopy. Sol State Comm, 1995, 93: 847-851

99 Weinstein BA, Piermarini GJ. Raman-scattering and phonon dispersion in $\mathrm{Si}$ and $\mathrm{GaP}$ at very high pressure. Phys Rev B, 1975, 12: 1172

100 Hartschuh A, Pedrosa HN, Novotny L, et al. Simultaneous fluorescence and Raman scattering from single carbon nanotubes. Science, 2003, 301: 1354-1356

101 Anastassakis E. Angular dispersion of optical phonon frequencies in strained cubic crystals. J Appl Phys, 1997, 82: 1582-1591

102 Reithmaier JP, Hoger R, Riechert H, et al. Band offset in elastically strained InGaAs/GaAs multiple quantum wells determined by optical absorption and electronic Raman scattering. Appl Phys Lett, 1990, 56: 536-538

103 Spitzer J, Ruf T, Cardona M, et al. Raman-scattering by optical phonons in isotopic ${ }^{70} \mathrm{Ge}_{n}{ }^{74} \mathrm{Ge}_{n}$ superlattices. Phys Rev Lett, 1994, 72: 1565

104 Pinczuk A, Abstreiter G, Trommer R, et al. Raman scattering by wave vector dependent coupled plasmon LO phonons of n-GaAs. Sol State Comm, 1977, 21: 959-962

105 Pinczuk A, Abstreiter G, Trommer R, et al. Resonance enhancement of Raman scattering by electron-gas excitations of $n-G a A s$. Sol State Comm, 1979, 30: 429-432

106 Baumgartner M, Abstreiter G. Interaction between electronic and phonon Raman scattering in hole space-charge layers on silicon. Surf Sci, 1984, 142: 357-361

107 Schuller C, Biese G, Keller K, et al. Single-particle excitations and many particle interactions in quantum wires and dots. Phys Rev B, 1996, 54: 17304

108 Pauzauskie PJ, Talaga D, Seo K, et al. Polarized Raman confocal microscopy of single gallium nitride nanowires. J Am Chem Soc, 2005, 127: 17146

109 Long DA. Raman Spectroscopy Raman Spectroscopy. London: McGraw-Hill, 1977

110 Zardo I, Conesa-Boj S, Peiro F, et al. Raman spectroscopy of wurtzite and zinc-blende GaAs nanowires: polarization dependence, selection rules, and strain effects. Phys Rev B, 2009, 80: 245324

111 Cardona M, Güntherodt G (eds.). Light Scattering in Solids II: Basic concepts and Instrumentation. Berlin: Springer-Verlag, 1982

112 Richter W. Solid State Physics, Springer Tracts in Modern Physics. Berlin: Springer-Verlag, 1976

113 Abstreiter G, Cardona M, Pinczuk A. Light scattering by free carrier excitations in semiconductors. Top Appl Phys, 1984, 54: 5-150

114 Ketterer B, Heiss M, Uccelli E, et al. Untangling the electronic band structure of wurtzite GaAs nanowires by resonant Raman spectroscopy. ACS Nano, 2011, 5: 7585-7592

115 Kusch P, Breuer S, Ramsteiner M, et al. Band gap of wurtzite GaAs: a resonant Raman study. Phys Rev B, 2012, 86: 085317

116 Moeller M, Lima Jr MM, Cantarero A, et al. Polarized and resonant 
Raman spectroscopy on single InAs nanowires. Phys Rev B, 2011, 84: 085318

117 Grimsditch M, Cardona M, Calleja JM, et al. Resonance in the Raman scattering of $\mathrm{CaF}_{2}, \mathrm{SrF}_{2}, \mathrm{BaF}_{2}$, and diamond. J Raman Spectrosc, 1981, 10: 77-81

118 Müller EW, Panitz JA, McLane SB. The atom-probe field ion microscope. Rev Sci Instrum, 1968, 39: 83-86

119 Tsong T. Atom Probe Field Ion Microscopy Field Emission, and Surfaces and Interfaces at Atomic Resolution. Cambridge: Cambridge University Press, 1990

120 Seidman DN, Stiller K. An atom-probe tomography primer. MRS Bull, 2009, 34: 717-724.

121 Miller MK, Forbes R. Atom probe tomography. Mater Charact, 2009, 60: 461-469

122 Du S, Burgess T, Gault B, et al. Quantitative dopant distributions in GaAs nanowires using atom probe tomography. Ultramicroscopy, 2014, 132: 186-192

123 Perea DE, Lensch JL, May SJ, et al. Composition analysis of single semiconductor nanowires using pulsed-laser atom probe tomography. Appl Phys A, 2006, 85: 271-275

$124 \mathrm{Xu}$ T, Nys JP, Grandidier B, et al. Growth of Si nanowires on micropillars for the study of their dopant distribution by atom probe tomography. J Vac Sci Technol, 2008, 26: 1960-1963

125 Allen JE, Hemesath ER, Perea DE, et al. High-resolution detection of $\mathrm{Au}$ catalyst atoms in Si nanowires. Nat Nanotechnol, 2008, 3 : 168-173

126 Perea DE, Hemesath ER, Schwalbach EJ, et al. Direct measurement of dopant distribution in an individual vapour-liquid-solid nanowire. Nat Nanotechnol, 2009, 4: 315-319

127 Riley JR, Bernal RA, Li Q, et al. Atom probe tomography of a-axis GaN nanowires: analysis of nonstoichiometric evaporation behavior. ACS Nano, 2012, 6: 3898-3906

128 Zhang G, Tateno K, Gotoh H, et al. Compositional, and optical characterizations of vertically aligned $\mathrm{AlAs} / \mathrm{GaAs} / \mathrm{GaP}$ heterostructure nanowires epitaxially grown on Si substrate. Jpn J Appl Phys, 2010, 49: 015001

129 Shtrikman H, Popovitz-Biro R, Huth P, et al. Core-shell GaAs-AlAs nanowires grown by MBE. In Nanoelectronics Conference (INEC), 2010 3rd International, Hongkong, 2010: 103-104

130 Chen C, Shehata S, Fradin C, et al. Self-directed growth of AlGaAs core-shell nanowires for visible light applications. Nano Lett, 2007, 7: 2584-2589

131 Lucot D, Jabeen F, Harmand JC, et al. Quasi one-dimensional transport in single GaAs/AlGaAs core-shell nanowires. Appl Phys Lett, 2011, 98: 142114

132 Parkinson P, Joyce HJ, Gao Q, et al. Carrier lifetime and mobility enhancement in nearly defect-free core-shell nanowires measured using time-resolved terahertz spectroscopy. Nano Lett, 2009, 9: 3349-3353

133 Persano A, Nabet B, Taurino A, et al. A polarization anisotropy of individual core/shell GaAs/AlGaAs nanowires by photocurrent spectroscopy. Appl Phys Lett, 2011, 98: 153106

134 Demichel O, Heiss M, Bleuse J, et al. Impact of surfaces on the optical properties of GaAs nanowires. Appl Phys Lett, 2010, 97: 201907

135 Gallo EM, Chen G, Currie M, et al. Picosecond response times in GaAs/AlGaAs core/shell nanowire-based photodetectors. Appl Phys Lett, 2011, 98: 241113

136 Kang JH, Gao Q, Joyce HJ, et al. Novel growth and properties of GaAs nanowires on Si substrates. Nanotechnology, 2010, 21: 035604

137 Persano A, Taurino A, Prete P, et al. Photocurrent properties of single GaAs/AlGaAs core-shell nanowires with Schottky contacts. Nanotechnology, 2012, 23: 4657

138 De A, Pryor CE. Predicted band structures of III-V semiconductors in the wurtzite phase. Phys Rev B, 2010, 81: 155210

139 Spirkoska D, Morral A, Dufouleur J, et al. Free standing modulation doped core-shell GaAs/AlGaAs hetero-nanowires. Phys Status Solidi Rapid Res Lett, 2011, 5: 353-355

140 Zhou HL, Hoang TB, Dheeraj DL, et al. Wurtzite GaAs/AlGaAs core-shell nanowires grown by molecular beam epitaxy. Nanotechnology, 2009, 20: 415701

141 Yeh CY, Wie SH, Zunger A. Relationships between the band gaps of the zinc-blende and wurtzite modifications of semiconductors. Phys Rev B, 1994, 50: 2715

142 Li A, Ercolani D, Lugani L, et al. Synthesis of AlAs and AlAs-GaAs core-shell nanowires. Cryst Growth Des, 2011, 11: 4053-4058

143 Funk S, Li A, Ercolani D, et al. Crystal phase induced bandgap modifications in AlAs nanowires probed by resonant Raman spectroscopy. ACS Nano, 2013, 7: 1400-1407

144 Gudiksen MS, Lauhon LJ, Wang J, et al. Growth of nanowire superlattice structures for nanoscale photonics and electronics. Nature, 2002, 415: 617-620

145 Mohseni PK, Rodrigues AD, Galzerani JC, et al. Structural and optical analysis of GaAsP/GaP core-shell nanowires. J Appl Phys, 2009, 106: 124306

146 Borgström MT, Verheijen MA, Immink G, et al. Interface study on heterostructured GaP-GaAs nanowires. Nanotechnology, 2006, 17: 4010

147 Borgström MT, Zwiller V, Müller E, et al. Optically bright quantum dots in single nanowires. Nano Lett, 2005, 5: 1439-1443

148 Zhang G, Tateno K, Sogawa T, et al. Vertically aligned GaP/GaAs Core-multishell nanowires epitaxially grown on Si substrate. Appl Phys Express, 2008, 1: 064003

149 Shin JC, Kim KH, Yu KJ, et al. InxGa ${ }_{1-x}$ As nanowires on silicon: one-dimensional heterogeneous epitaxy, bandgap engineering, and photovoltaics. Nano Lett, 2011: 4831-4838

150 Moewe M, Chuang LC, Crankshaw S, et al. Core-shell InGaAs/GaAs quantum well nanoneedles grown on silicon with silicon-transparent emission. Optics express, 2009, 17: 7831-7836

151 Chen R, Tran TTD, Ng KW, et al. Nanolasers grown on silicon. Nat Photon, 2011, 5: 170-175

152 Scofield Adam C, Kim Se Heon, Shapiro Joshua N, et al. Bottom-up photonic crystal lasers. Nano Lett, 2011, 11: 5387-5390

153 Yang T, Hertenberger S, Morkötter S, et al. Size, composition, and doping effects on $\operatorname{In}(\mathrm{Ga})$ As nanowire/Si tunnel diodes probed by conductive atomic force microscopy. Appl Phys Lett, 2012, 101: 1-6

154 Tomioka K, Yoshimura M, Fukui T. A III-V nanowire channel on silicon for high-performance vertical transistors. Nature, 2012, 488: 189-192

155 Tatebayashi J, Ota Y, Ishida S, Nishioka M, et al. Site controlled formation of InAs/GaAs quantum-dot-in-nanowires for single photon emitters. Appl Phys Lett, 2012, 100: 1-5

156 Koblmüller G, Abstreiter G. Growth and properties of InGaAs nanowires on silicon. Phys Status Solidi R, 2014, 8: 11-30

157 Kim Y, Joyce HJ, Gao Q, et al. Influence of nanowire density on the shape and optical properties of ternary InGaAs nanowires. Nano Lett, 2006, 6: 599-604

158 Regolin I, Khorenko V, Prost W, et al. Composition control in metal-organic vapor-phase epitaxy grown InGaAs nanowhiskers. J Appl Phys, 2006, 100: 4-9

159 Bauer J, Gottschalch V, Paetzelt H, et al. VLS growth of GaAs/(InGa) As/GaAs axial double-heterostructure nanowires by MOVPE. J Cryst Growth, 2008, 310: 5106-5110

160 Paladugu M, Zou J, Guo YN, et al. Polarity driven formation of InAs/GaAs hierarchical nanowire heterostructures. Appl Phys Lett. 2008, 93: 201908

161 Guo YN, Burgess T, Gao Q, et al. Polarity-driven nonuniform com- 
position in InGaAs nanowires, Nano Lett, 2013, 13: 5085-5089

162 Shin JC, Choi KJ, Kim DY, et al. Characteristics of strain-induced $\mathrm{In}_{x} \mathrm{Ga}_{1-x} \mathrm{As}$ nanowires grown on $\mathrm{Si}(111)$ substrates. Crystal Growth Design, 2012, 12: 2994-2998

163 Hertenberger S, Funk S, Vizbaras K, et al. High compositional homogeneit in In-rich InGaAs nanowire arrays on nanoimprinted $\mathrm{SiO}_{2} / \mathrm{Si}$ (111). Appl Phys Lett, 2012, 101: 043116

164 Morkötter S, Funk S, Liang M, et al. Role of microstructure on optical properties in high-uniformity $\mathrm{In}_{1-x} \mathrm{Ga}_{x}$ As nanowire arrays: evidence of a wider wurtzite band gap. Phys Rev B, 2013, 87: 205303

165 Björk MT, Ohlsson BJ, Sass T, et al. One-dimensional heterostructures in semiconductor nanowhiskers. Appl Phys Lett, 2002, 80: 1058-1060

166 Björk MT, Ohlsson BJ, Thelander C, et al. One-dimensional steeplechase for electrons realized. Nano Lett, 2002, 2: 87-89

167 Björk MT, Ohlsson BJ, Thelander C, et al. Nanowire resonant tunneling diodes. Appl Phys Lett, 2002, 81: 4458

168 Roddaro S, Pescaglini A, Ercolani D, et al. Manipulation of electron orbitals in hard-wall InAs/InP nanowire quantum dots. Nano Lett, 2011, 11: 1695-1699

169 Yazawa M, Koguchi M, Hiruma K. Heteroepitaxial ultrafine wirelike growth of InAs on GaAs substrates. Appl Phys Lett, 1991, 58: 1080-1082

170 Popovitz-Biro R, Kretinin A, Von Huth P, et al. InAs/GaAs coreshell nanowires. Cryst Growth Des, 2011, 11: 3858-3865

171 Takahash K, Morizumi K. Growth of InAs whiskers inwurtzite structure. Jpn J Appl Phys, 1966, 5: 657-662

172 Yeh CY, Lu ZW, Froyen S, et al. Zinc-blende-wurtzite polytypism in semiconductors. Phys Rev B, 1992, 46: 10086

173 Duan XF, Huang Y, Y Cui, et al. Indium phosphide nanowires as building blocks for nanoscale electronic and optoelectronic devices. Nature, 2001, 409: 66-69

174 Wang J, Gudiksen MS, Duan X, et al. Highly polarized photoluminescence and photodetection from single indium phosphide nanowires. Science, 2001, 293: 1455-1457

175 Ding Y, Motohisa J, Hua B, et al. Observation of microcavity modes and waveguides in InP nanowires fabricated by selective-area metalorganic vapor-phase epitaxy. Nano Lett, 2007, 7: 3598-3602

176 Choi SJ, Djordjev K, Sang Jun C, et al. Ion beam irradiation induced fabrication of vertical coupling photonic structures. IEEE Photonics Technol Lett, 2004, 16: 828-830

177 Choi SJ, Zhen P, Qi Y, et al. A high-Q wavelength filter based on buried heterostructure ring resonators integrated with a semiconductor optical amplifier. IEEE Photonics Technol Lett, 2005, 17: 2101-2103

178 Sadagopan T, Choi SJ, Sang JC, et al. High-speed, low-voltage modulation in circular WGM microresonators. IEEE Photonics Technol Lett, 2005, 16: 414-416

179 Mino ED, Kelkensberg F, Kouwen M, et al. Single quantum dot nanowire LEDs. Nano Lett, 2007, 7: 367-371

180 Mohan P, Motohisa J, Fukui T. Fabrication of InP/InAs/InP core-multishell heterostructure nanowires by selective area metalorganic vapor phase epitaxy. Appl Phys Lett, 2006, 88: 1-4

181 Mohan P, Motohisa J, Fukui T. Realization of conductive InAs nanotubes based on lattice-mismatched InP/InAs core-shell nanowires. Appl Phys Lett, 2006, 88: 87-90

182 Kawaguchi K, Sudo H, Matsuda M, et al. Radial InP/InAsP/InP heterostructure nanowires on patterned Si substrates using self-catalyzed growth for verticaltype optical devices. Appl Phys Lett, 2015, 106: 012107

183 Bhunia S, Kawamura T, Fujikawa S, et al. Vapor-liquid-solid growth of vertically aligned InP nanowires by metalorganic vapor phase epitaxy. Thin Solid Films, 2004, 464: 244-247
184 Xiong Q, Wang J, Eklund PC. Coherent twinning phenomena: towards twinning superlattices in III-V semiconducting nanowires. Nano Lett, 2006, 6: 2736-2742

185 Bao J, Bell DC, Capasso F, et al. Optical properties of rotationally twinned InP nanowire heterostructures. Nano Lett, 2008, 8: 836841

186 Ikejiri K, Kitauchi Y, Tomioka K, et al. Zinc blende and wurtzite crystal phase mixing and transition in indium phosphide nanowires. Nano Lett, 2011, 11: 4314-4318

187 Akopian N, Patriarche G, Liu L, et al. Crystal phase quantum dots. Nano Lett, 2010, 10: 1198-1201

188 Ikonic Z, Srivastava GP, Inkson JC. Optical properties of twinning superlattices in diamond-type and zinc-blende type semiconductors. Phys Rev B, 1995, 52: 14078-14085

189 Joyce J, Yong CK, Docherty CJ, et al. Ultralow surface recombination velocity in InP nanowires probed by terahertz spectroscopy. Nano Lett, 2012, 12: 5325-5330

190 Wallentin J, Ek M, Wallenberg LR, et al. Electron trapping in InP nanowire FETs with stacking faults. Nano Lett, 2012, 12: 151-155

191 Krishnamachari U, Borgström M, Ohlsson BJ, et al. Defect-free InP nanowires grown in [001] direction on $\operatorname{InP}(001)$. Appl Phys Lett, 2004, 85: 2077-2079

192 Zhang Y, Williamson F. Evaluation of an InSb infrared detector at liquid $\mathrm{N}_{2}$ and liquid He temperatures. Appl Opt, 1982, 21: 20362040

193 Kornreich P, Walsh L, Flattery J, et al. Proposed size-effect high-electronmobility transistor. Solid State Electron, 1986, 29: 421-427

194 Ashley T, Dean A, Elliott C, et al. Uncooled highspeed InSb field-effect transistors. Appl Phys Lett, 1995, 66: 481-483

195 Caroff P, Messing ME, Borg BM, et al. InSb heterostructure nanowires: MOVPE growth under extreme lattice mismatch. Nanotechnology, 2009, 20: 495606

196 Mourik V, Zuo K, Frolov SM, et al. Signatures of majorana fermions in hybrid superconductor-semiconductor nanowire devices. Science, 2012, 336: 1003-1007

197 Das A, Ronen Y, Most Y, et al. Zero-bias peaks and splitting in an Al-InAs nanowire topological superconductor as a signature of Majorana fermions. Nat Phys, 2012, 8: 887-895

198 Heremans J, Partin D, Thrush C, et al. Narrow-gap semiconductor magnetic-field sensors and applications. Semicond Sci Technol, 1993, 8: S424-S430

199 Chung SJ, Goldammer KJ, Lindstrom SC, et al. Study of factors limiting electron mobility in InSb quantum wells. J Vac Sci Technol B, 1999, 17: 1151

200 Nilsson HA, Caroff P, Thelander C, et al. Giant, level-dependent g factors in InSb nanowire quantum dots. Nano Lett, 2009, 9: 31513156

201 Mingo N. Thermoelectric figure of merit and maximum power factor in III-V semiconductor nanowires. Appl Phys Lett, 2004, 84: 2652

202 Mingo N. Erratum: "Thermoelectric figure of merit and maximum power factor in III-V semiconductor nanowires". Appl Phys Lett, 2006, 88: 149902

203 Philipose U, Sapkota G, Salfi J, et al. Influence of growth temperature on the stoichiometry of InSb nanowires grown by vapor phase transport. Semic Sci Technol, 2010, 25: 075004

204 Vogel AT, Boor J, Becker M, et al. Ag-assisted CBE growth of ordered InSb nanowire arrays. Nanotechnology, 2011, 22: 015605

205 Nilsson HA, Samuelsson L, Caroff P, et al. Supercurrent and multiple andreev reflections in an InSb nanowire Josephson junction. Nano Lett, 2012, 12: 228-233

206 Pitanti A, Ercolani D, Sorba L, et al. InAs/InP/InSb nanowires as low capacitancen-n heterojunction diodes. Phys Rev X, 2011, 1: 
011006

207 Chen CY, Shik A, Pitanti A, et al. Electron beam induced current in InSb-InAs nanowire type-III heterostructures. App Phys Lett, 2012, 101: 063116

208 Pitanti A, Coquillat D, Ercolani D, et al. Terahertz detection by heterostructed InAs/InSb nanowire based field effect transistors. App Phys Lett, 2012, 101: 141103

209 Plissard SR, Slapak DR, Verheijen MA, et al. From InSb nanowires to nanocubes: looking for the sweet spot. Nano Lett, 2012, 12: 1794-1798

210 Dubrovskii VG, Sibirev NV, Suris RA, et al. The role of surface diffusion of adatoms in the formation of nanowire crystals. Semiconductors, 2006, 40: 1075-1082

211 Kim Y, Joyce HJ, Gao Q, et al. Influence of nanowire density on the shape and optical properties of ternary InGaAs nanowires. Nano Lett, 2006, 6: 599-604

212 Dubrovskii VG, Sibirev NV. Growth rate of a crystal facet of arbitrary size and growth kinetics of vertical nanowires. Phys Rev E, 2004, 70: 031604

213 Lugani L, Ercolani D, Sorba L, et al. Modeling of InAs-InSb nanowires grown by $\mathrm{Au}$-assisted chemical beam epitaxy. Nanotechnology, 2012, 23: 095602

214 Abramson AR, KimWC, Huxtable ST, et al. Fabrication and characterization of a nanowire/polymer-based nanocomposite for a prototype thermoelectric device. Microelectromech Syst, 2004, 13 : 505-513

215 Keyani J, Stacy AM, Sharp J. Assembly and measurement of a hybrid nanowire-bulk thermoelectric device. Appl Phys Lett, 2006, 89: 233106

216 Li A, Sibirev NV, Ercolani D, Dubrovskii VG, Sorba L. Readsorption assisted growth of InAs/InSb heterostructured nanowire arrays. Cryst Growth Des, 2013, 13: 878-882

217 Plissard R, van Weperen I, Car D, et al. Formation and electronic properties of InSb nanocrosses. Nat Nanotechnol, 2013, 8: 859-864

218 Car D, Verheijen MA, Wang J, et al. Rationally designed single-crystalline nanowire networks. Adv Mater, 2014, 26: 4875-4879

219 Liu X, Lin Y, Zhou S, et al. Complex nanostructures: synthesis and energetic applications. Energies, 2010, 3: 285-300

220 Woolley JC, Warner J. Optical energy-gap variaton in InAs-InSb alloys. Can J Phys, 1964, 42: 1879-1885

221 Stringfellow GB, Greene PE. Liquid phase epitaxial growth of InAs $s_{1-x}$ $\mathrm{Sb}_{x}$. J Electrochem Soc, 1971, 118: 805-810

222 Dobbelaere W, Boeck JD, Borghs G. Growth and optical characterization of $\operatorname{InAs}_{1-x} \mathrm{Sb}_{x}(0 \leqslant x \leqslant 1)$ on GaAs and on GaAs-coated Si by molecular beam epitaxy. Appl Phys Lett, 1989, 55: 1856-1858

223 Roddaro S, Pescaglini A, Ercolani D, et al. Hot-electron effects in InAs nanowire Josephson junctions. Nano Res, 2011, 4: 259-272

224 Vurgaftman I, Meyer JR, Ram-Mohan LR. Band parameters for III-V compound semiconductors and their alloys. J Appl Phys,
2001, 89: 5815

225 Borg BM, Dick KA, Eymery J, Wernersson LE. Enhanced Sb incorporation in InAsSb nanowires grown by MOVPE. Appl Phys Lett, 2011, 98: 113104

226 Ercolani D, Gemmi M, Nasi L, et al. Growth of InAs/InAsSb heterostructured nanowires. Nanotechnology, 2012, 23: 115606

227 Pea M, Ercolani D, Li a, et al. Suppression of lateral growth in InAs/ InAsSb heterostructured nanowires. J Crystal Growth, 2013, 366: $8-14$

228 Anyebe EA, Rajpalke MK, Veal TD, et al. Surfactant effect of antimony addition to the morphology of self-catalyzed $\mathrm{InAs}_{1-x} \mathrm{Sb}_{x}$ nanowires. Nano Res, 2014, 4: 1-11

229 Du WN, Yang XG, Wang XY, et al. The self-seeded growth of InAsSb nanowires on silicon by metal-organic vapor phase epitaxy. J Crystal Growth, 2014, 396: 33-37

230 Zhuang QD, Anyebe EA, et al. Sb-induced phase control of InAsSb nanowires grown by molecular beam epitaxy. Nano Lett, 2015, 15: 1109-1116

231 Bierman MJ, Jin S. Potential applications of hierarchical branching nanowires in solar energy conversion. Energy Environmental Sci, 2009, 2: 1050-1059

232 Wang D, Qian F, Yang C, Zhong Z, Lieber CM. Rational growth of branched and hyper-branched nanowire structures. Nano Lett, 2004, 4: 871-874

233 Dick KA, Deppert K, Larsson MW, et al. Synthesis of branched "nanotrees" by controlled seeding of multiple branching events. Nat mater, 2004, 3: 380-384

234 Sun K, Jing Y, Li C, et al. 3D branched nanowire heterojunction photoelectrodes for high-efficiency solar water splitting and $\mathrm{H}_{2}$ generation. Nanoscale, 2012, 4: 1515-1521

235 Yan C, Li X, Zhou K, et al. Heteroepitaxial growth of GaSb nanotrees with an ultra-low reflectivity in a broad spectral range. Nano Lett, 2012, 12: 1799-1805

236 Kargar A, Sun K, Jing Y, et al. 3D branched nanowire photoelectrochemical electrodes for efficient solar water splitting. ACS Nano, 2013, 7: 9407-9415

237 Adachi S. Properties of Semiconductor Alloys: Group-IV, III-V and II-VI Semiconductors. New York: John Wiley \& Sons, 2009

Acknowledgement This work was supported by the National Natural Science Foundation of China (11327901 and 11127404), and the Project of Construction of Innovative Teams and Teacher Career Development for Universities and Colleges Under Beijing Municipality (IDHT20140504).

Author contributions $\mathrm{Li}$ A is responsible for section II, III, IV, V, and VI. Li A and Zou J contribute section IV, V, VI, and VII. Han X, and Zou $\mathrm{J}$ contribute for section V, VI, and VII. All authors involve in writing and refinement of the manuscript.

Conflict of interest The authors declare that they have no conflict of interests. 


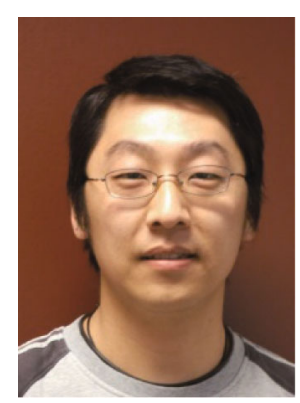

Ang Li received his PhD degree (cum laude) in condensed matter physics from Scuola Normale Superiore di Pisa. He joined Technical University of Eindhoven and Delft University of Techonology as a postdoctoral researcher in 2013, as well as a guest researcher in Philips Innovation Centre. From the end of 2015, he joined the Institute of Microstructure and Property of Advanced Materials of Beijing University of Technology. His research interests include the growth of low dimensional semiconductor materials, semiconductor-based quantum devices fabrication, and advanced electron microcopy.

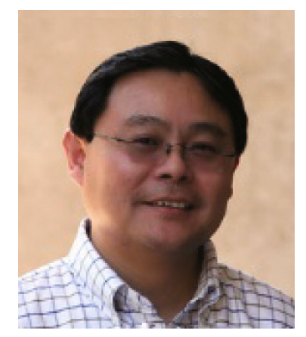

Jin Zou is a professor shared between the School of Mechanical and Mining Engineering (Materials Engineering) and the Centre for Microscopy and Microanalysis, at the University of Queensland. Professor Zou's research interest focuses on the understanding of the evolution of advanced, smart and nano-scaled materials and the understanding of fundamental properties of these materials through correlating their structures with their demonstrated properties. He has more than 500 SCI publications, most of them published in leading international journals.

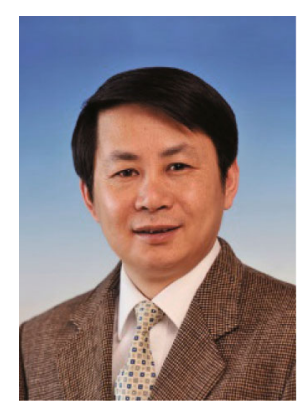

Xiaodong Han currently works as a professor at the Institute of Microstructure and Property of Advanced Materials of Beijing University of Technology. His research interest focuses on developing novel electron microscopy tools and instruments for atomic scale and multi-scale in situ experiments on structural and functional materials, including high strength yet ductile structural materials, energy related materials, catalysis materials, atomic scale understanding solid-solid, liquid-solid and gas-solid reactions etc. He has published more than 150 papers at high impact journals such as Science, Nature Communications, Nano Letters, Physical Review Letters and Acta Materialia.

\title{
III-V族半导体与其异质结纳米线的生长
}

\author{
李昂, 邹进, 韩晓东
}

摘要 这篇综述有针对性地对近几年III-V族化合物半导体和其异质结纳米线的生长进行了总结. 首先详尽地介绍了单质和异质结III-V纳米线 的生长机理, 并且讨论了它们常见的晶体结构. 然后总结了常见的III-V族纳米线的生长条件和工艺要点, 包括生长温度, III/V 比例, 异质结材料 和界面等对纳米线形貌和结构的影响. 同时这篇综述也着重介绍了最新发展的表征纳米线晶体结构和物理性能的新技术, 如: 先进电子显微 学、电子衍射、显微拉曼光谱原子探针断层扫描等. 最后对纳米线研究和应用的未来发展提出了展望. 